Cochrane Database of Systematic Reviews

\title{
Remote ischaemic conditioning for preventing and treating ischaemic stroke (Review)
}

Zhao W, Zhang J, Sadowsky MG, Meng R, Ding Y, Ji X

Zhao W, Zhang J, Sadowsky MG, Meng R, Ding Y, Ji X.

Remote ischaemic conditioning for preventing and treating ischaemic stroke.

Cochrane Database of Systematic Reviews 2018, Issue 7. Art. No.: CD012503.

DOI: 10.1002/14651858.CD012503.pub2.

www.cochranelibrary.com 
TABLE OF CONTENTS

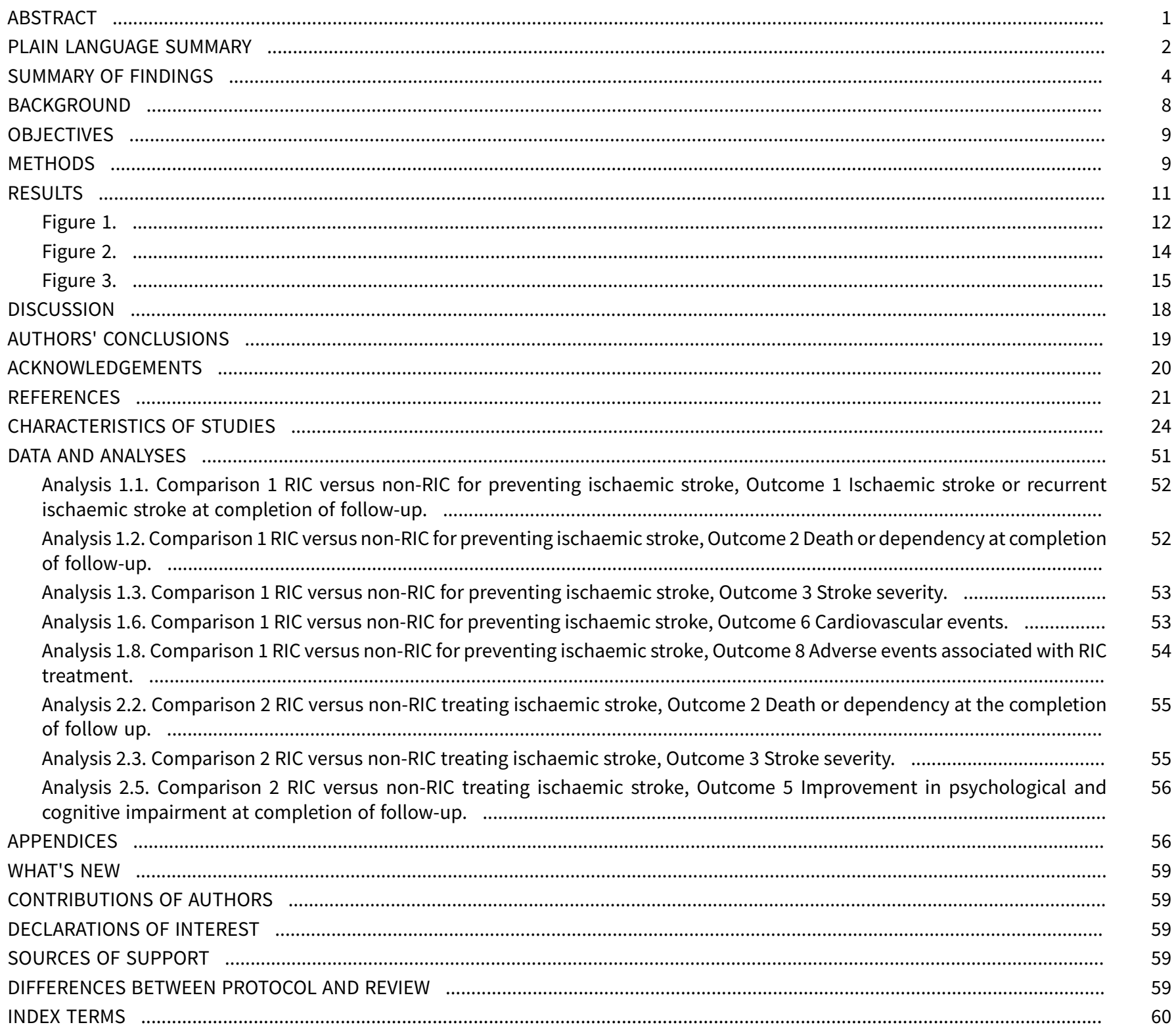


[Intervention Review]

\section{Remote ischaemic conditioning for preventing and treating ischaemic stroke}

Wenbo Zhao ${ }^{1}$, Jing Zhang ${ }^{1}$, Mordechai G Sadowsky², Ran Meng1, Yuchuan Ding², Xunming Ji3

1Department of Neurology, Xuanwu Hospital, Capital Medical University, Beijing, China. 2Department of Neurological Surgery, Wayne State University School of Medicine, Detroit, Michigan, USA. ${ }^{3}$ Department of Neurosurgery, Xuanwu Hospital, Capital Medical University, Beijing, China

Contact: Xunming Ji, Department of Neurosurgery, Xuanwu Hospital, Capital Medical University, Beijing, 100053, China. jixm@ccmu.edu.cn.

Editorial group: Cochrane Stroke Group.

Publication status and date: Edited (no change to conclusions), published in Issue 9, 2019.

Citation: Zhao W, Zhang J, Sadowsky MG, Meng R, Ding Y, Ji X. Remote ischaemic conditioning for preventing and treating ischaemic stroke. Cochrane Database of Systematic Reviews 2018, Issue 7. Art. No.: CD012503. DOI: 10.1002/14651858.CD012503.pub2.

Copyright @ 2019 The Cochrane Collaboration. Published by John Wiley \& Sons, Ltd.

\section{A B S T R A C T}

\section{Background}

Remote ischaemic conditioning (RIC) has been developed as a neuroprotective strategy to prevent and treat ischaemic stroke. It usually involves restricting blood flow to limbs and then releasing the ischaemic blood to promote a neuroprotective effect. Preclinical studies have suggested that RIC may have beneficial effects in ischaemic stroke patients and those at risk of ischaemic stroke. However, existing evidence is insufficient to demonstrate the efficacy and safety of RIC in preventing and treating ischaemic stroke.

\section{Objectives}

To assess the benefits and harms of RIC for preventing ischaemic stroke and for treating people with ischaemic stroke and those at risk for ischaemic stroke.

\section{Search methods}

We searched the Cochrane Stroke Group Trials Register (16 January 2018), the Cochrane Central Register of Controlled Trials (CENTRAL; 2017, Issue 12) in the Cochrane Library (January 2018), MEDLINE Ovid (1946 to January 2018), Embase Ovid (1974 to January 2018), Web of Science Core Collection (1950 to January 2018) and three Chinese databases (January 2018). We also searched four ongoing trials registers, reference lists, and conference proceedings.

\section{Selection criteria}

We included randomised controlled trials (RCTs) comparing RIC with sham RIC or medical management in people with ischaemic stroke or at risk of ischaemic stroke.

\section{Data collection and analysis}

Two review authors independently selected studies, assessed trial quality and risk of bias, and extracted data. We used the GRADE approach to assess the quality of the evidence.

\section{Main results}

We included seven trials, involving 735 participants, in this review. We analysed the effects of RIC on preventing and treating ischaemic stroke respectively. 
We evaluated risk of bias and judged it to be low for generation of allocation sequence in six studies and unclear in one study; unclear for allocation concealment in four studies and low in three studies; high for incomplete outcome data (attrition bias) in five studies and low in two studies; high for blinding in three studies and low in four studies; low for selective reporting; and high for other sources of bias in six studies and low in one study.

We included three trials (involving 371 participants) in the analysis of the effects of RIC on ischaemic stroke prevention. In people with symptomatic intracerebral artery stenosis, recurrent stroke was significantly reduced by RIC (risk ratio (RR) $0.32,95 \%$ confidence interval (CI) 0.12 to 0.83 ; 2 trials, 182 participants, low-quality evidence). In people with carotid stenosis undergoing carotid stenting, there was no significant difference in the incidence of ischaemic stroke between participants treated with RIC and non-RIC (RR $0.22,95 \% \mathrm{Cl} 0.01$ to 4.03 ; 1 trial, 189 participants, low-quality evidence); however the stroke severity (assessed by infarct volume) was significantly lower in participants treated with RIC (mean difference (MD) $-0.17 \mathrm{~mL}, 95 \% \mathrm{Cl}-0.23$ to $-0.11 ; 1$ trial, 189 participants, low-quality evidence). Adverse events associated with RIC were significantly higher in participants treated with RIC (RR 10.91; $95 \%$ Cl 2.01 to $59.28 ; 3$ trials, 371 participants, low-quality evidence), but no severe adverse event was attributable to RIC treatment. No participants experienced death or cardiovascular events during the period of the studies; and no trial reported haemorrhagic stroke or improvement in neurological, phycological or cognitive impairment.

We included four trials (involving 364 participants) in the analysis of the effects of RIC on ischaemic stroke treatment. In acute ischaemic stroke, for people receiving intravenous thrombolysis, the rate of death or dependency was significantly increased by RIC treatment compared with non-RIC treatment (RR 2.34; 95\% 1.19 to 4.61; 1 trial, 285 participants, low-quality evidence). In people with acute ischaemic stroke, there was no significant difference between RIC and non-RIC for reducing stroke severity as assessed by the National Institutes of Health Stroke Scale score and the final infarct volume (standardised mean difference (SMD) $-0.24 \mathrm{~mL}, 95 \% \mathrm{Cl}-1.02$ to $0.54 ; 2$ trials, 175 participants, very low quality evidence). There was no significant difference between RIC and non-RIC for improving the psychological impairment (SMD -0.37 points, $95 \% \mathrm{Cl}-1.15$ to $0.41 ; 1$ trial, 26 participants, very low quality evidence) and the cognitive impairment (SMD -0.26 points; $95 \% \mathrm{Cl}-0.72$ to $0.21 ; 3$ trials, 79 participants, low-quality evidence) in people with acute ischaemic stroke and cerebral small vessel disease. No trial reported ischaemic stroke, recurrent ischaemic stroke, improvement in neurological impairment, hemorrhagic stroke, cardiovascular events, and RIC associated adverse events.

\section{Authors' conclusions}

We found low-quality evidence that RIC may reduce the risk of recurrent stroke in participants with intracerebral artery stenosis and reduce stroke severity in participants undergoing carotid stenting, but it may increase death or dependence in participants with acute ischaemic stroke who are undergoing intravenous thrombolysis. However, there is considerable uncertainty about these conclusions because of the small number of studies and low quality of the evidence.

\section{PLAIN LANGUAGE SUMMARY}

\section{Remote ischaemic conditioning for preventing and treating stroke caused by low blood flow}

\section{Review question}

What are the benefits of using remote ischaemic conditioning (a noninvasive physical therapy that involves inflating blood pressure cuffs to reduce blood flow in arms and legs, and then releasing the affected blood around the body) in people with stroke or those at risk of stroke caused by low blood flow?

\section{Background}

Stroke is the leading cause of adult disability globally, and ischaemic stroke (caused by reduced blood flow) accounts for most strokes. Nearly a quarter of individuals with ischaemic stroke will experience further events (recurrent stroke). Remote ischaemic conditioning (RIC) is a strategy to protect and prevent damage to brain tissue by improving its ability to tolerate reduced blood flow. Studies have suggested that RIC may have beneficial effects for preventing and treating ischaemic stroke.

\section{Study characteristics}

This review included seven studies (specifically randomised controlled trials), involving 735 people. The studies compared RIC with sham $\mathrm{RIC}$ or medical management in people with ischaemic stroke or at risk of ischaemic stroke. Three trials (involving 371 people) were eligible for our analysis of RIC for preventing ischaemic stroke, and another four trials (involving 364 people) were eligible for our analysis of RIC for treating ischaemic stroke. The included trials were carried out in China, Denmark, and the UK.

\section{Key results}

The results of this review are current up to January 2018. In people with narrowing of arteries in the brain, RIC may reduce the risk of recurrent stroke. In people being treated with stenting (the insertion of a metal or plastic tube) for narrowed arteries in the neck, RIC may reduce the size of new brain injuries caused by reduced blood flow. However, its effect on clinical outcomes (stroke and death) was unclear. Adverse events were significantly more common in the RIC group but were not reported to be severe. 
Among people with acute ischaemic stroke (where it had only been several hours from symptom onset) who received clot-dissolving medicines, we found that RIC may increase the risk of death or dependency (needing help from others). We found no significant differences in the size of the final stroke. In people with acute ischaemic stroke and chronic blood vessel disease of the brain, RIC did not affect measures of nerve function, mood, or thinking ability.

\section{Quality of evidence}

There is low-quality evidence which suggests that RIC may help prevent recurrent stroke in people with narrowed arteries in the brain, and may increase death or dependency in people with acute ischaemic stroke who received clot dissolving medication. The evidence is less clear for reducing the volume of the stroke (size of brain lesion caused by low blood flow). Further research is likely to have an important impact on our confidence in these findings. 


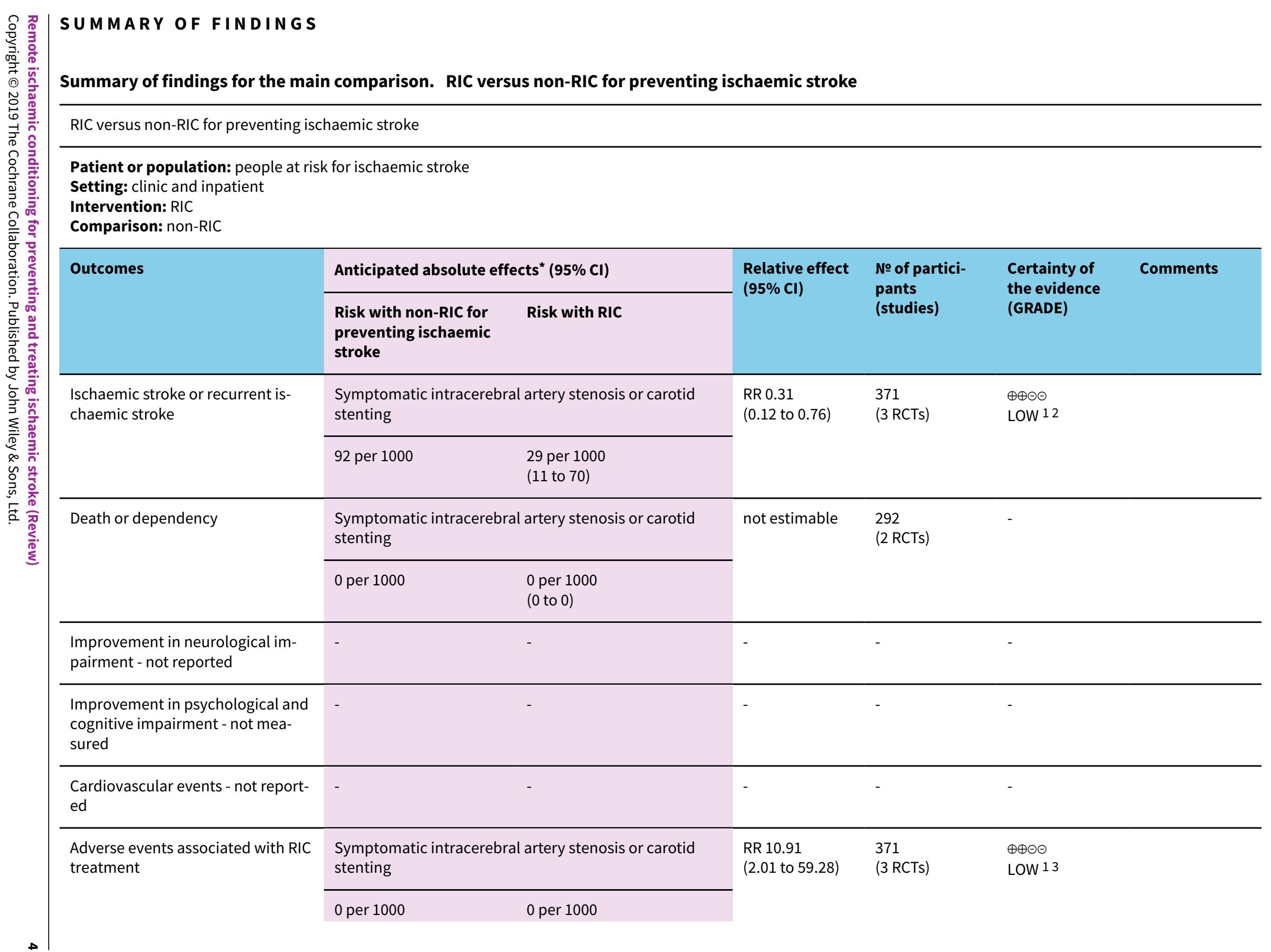


*The risk in the intervention group (and its $95 \%$ confidence interval) is based on the assumed risk in the comparison group and the relative effect of the intervention (and its $95 \% \mathrm{Cl})$.

CI: Confidence interval; RR: Risk ratio; OR: Odds ratio; RIC: remote ischaemic conditioning; RCT: randomised controlled trial;

\section{GRADE Working Group grades of evidence}

High certainty: We are very confident that the true effect lies close to that of the estimate of the effect

Moderate certainty: We are moderately confident in the effect estimate: The true effect is likely to be close to the estimate of the effect, but there is a possibility that it is substantially different

Low certainty: Our confidence in the effect estimate is limited: The true effect may be substantially different from the estimate of the effect

Very low certainty: We have very little confidence in the effect estimate: The true effect is likely to be substantially different from the estimate of effect

1 Downgrade by two levels because of very serious risk of bias and serious imprecision. Very serious risk of bias resulted from: participants were not blinded to the treatment protocol in Meng 2012 and Zhao 2017; Meng 2012 and Meng 2015 used per-protocol analysis methods; the corresponding author was one of the inventor of the RIC devices used in the studies; and there was large number of participants lost to follow up during the study period.

2 Serious imprecision. Incidence of ischaemic stroke was very lower in Zhao 2017, and the $\mathrm{Cl}$ for ischaemic stroke was wide.

3 Serious imprecision. Incidence of RIC-associated adverse events were very lower, and the CI for this outcome was very wide.

4 Downgrade by two levels because of serious risk of bias and serious indirectness. Very serious risk of bias results from: participants were not blinded to the treatment protocol; the corresponding author was one of the inventor of the RIC devices used; and there was large number of participants lost to follow up during the study period in Zhao 2017.

5 Serious indirectness. The infarct volume was used as a surrogate endpoint to assess stroke severity in Zhao 2017.

\section{Summary of findings 2 . RIC versus non-RIC treating ischaemic stroke}

RIC versus non-RIC for treating ischaemic stroke

Patient or population: people with ischaemic stroke

Setting: emergency, clinic and inpatient

Intervention: RIC

Comparison: non-RIC

\begin{tabular}{lllll}
\hline Outcomes & \begin{tabular}{l} 
Anticipated absolute effects ${ }^{*}(95 \% \mathrm{CI})$ \\
\cline { 2 - 5 }
\end{tabular} & $\begin{array}{l}\text { Relative effect } \\
(95 \% \mathrm{CI})\end{array}$ & $\begin{array}{l}\text { № of partici- } \\
\text { pants } \\
\text { (studies) } \\
\text { treating ischaemic } \\
\text { stroke }\end{array}$ & $\begin{array}{l}\text { Certainty of } \\
\text { the evidence } \\
\text { (GRADE) }\end{array}$ \\
\hline
\end{tabular}




\begin{tabular}{|c|c|c|c|c|c|}
\hline $\begin{array}{l}\text { Ischaemic stroke or recurrent ischaemic } \\
\text { stroke at completion of follow-up - not } \\
\text { measured }\end{array}$ & - & - & - & - & - \\
\hline Death or dependency & $\begin{array}{l}\text { Acute ischaemic stroke tr } \\
\text { thrombolysis }\end{array}$ & ated with intravenous & $\begin{array}{l}\text { RR } 2.34 \\
\text { (1.19 to } 4.61)\end{array}$ & $\begin{array}{l}285 \\
(1 \mathrm{RCT})\end{array}$ & $\begin{array}{l}\oplus \oplus \ominus \ominus \\
\text { LOW } 12\end{array}$ \\
\hline 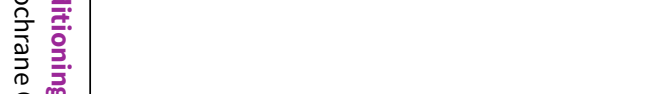 & 80 per 1000 & $\begin{array}{l}187 \text { per } 1000 \\
\text { (95 to } 369)\end{array}$ & & & \\
\hline $\begin{array}{l}\text { Improvement in neurological impair- } \\
\text { ment - not measured }\end{array}$ & - & - & - & - & - \\
\hline $\begin{array}{l}\text { Improvement in psychological and cog- } \\
\text { nitive impairment at completion of fol- } \\
\text { low-up }\end{array}$ & $\begin{array}{l}\text { The mean psychological } \\
\text { function was } 55.4 \text { points } \\
\text { and the mean cogni- } \\
\text { tive function was } 27.08 \\
\text { points. }\end{array}$ & $\begin{array}{l}\text { SMD } 0.29 \text { lower } \\
\text { (0.68 lower to } 0.11 \\
\text { higher) }\end{array}$ & - & $\begin{array}{l}105 \\
\text { (3 RCTs) }\end{array}$ & $\begin{array}{l}\oplus \oplus \ominus \ominus \\
\text { LOW } 34\end{array}$ \\
\hline Cardiovascular events - not reported & - & - & - & - & - \\
\hline $\begin{array}{l}\text { Adverse events associated with RIC } \\
\text { treatment - not reported }\end{array}$ & - & - & - & - & - \\
\hline Stroke severity & - & $\begin{array}{l}\text { SMD } 0.24 \text { lower } \\
\text { (1.02 lower to } 0.54 \\
\text { higher) }\end{array}$ & - & $\begin{array}{l}175 \\
\text { (2 study) }\end{array}$ & $\begin{array}{l}\oplus \odot \ominus \ominus \\
\text { VERY LOW } 456\end{array}$ \\
\hline
\end{tabular}

${ }^{*}$ The risk in the intervention group (and its $95 \%$ confidence interval) is based on the assumed risk in the comparison group and the relative effect of the intervention (and its $95 \% \mathrm{Cl})$.

Cl: Confidence interval; RR: Risk ratio; OR: Odds ratio; RIC: remote ischaemic conditioning; SMD: standardised mean difference; RCT: randomised controlled trial;

\section{GRADE Working Group grades of evidence}

High certainty: We are very confident that the true effect lies close to that of the estimate of the effect

Moderate certainty: We are moderately confident in the effect estimate: The true effect is likely to be close to the estimate of the effect, but there is a possibility that it is substantially different

Low certainty: Our confidence in the effect estimate is limited: The true effect may be substantially different from the estimate of the effect

Very low certainty: We have very little confidence in the effect estimate: The true effect is likely to be substantially different from the estimate of effect

1 Downgrade two levels by very serious risk of bias and serious imprecision. High risk of bias resulted from the unclear of allocation concealment, the unblinded treatment protocol to the participants, and large number of participants lost to follow up during the study period in Hougaard 2014.

2 Serious imprecision. There was a large number of participants excluded from the study after randomising in Hougaard 2014. 
Downgrade two levels by very serious risk of bias and serious inconsistency. Very serious risk of bias resulted from the unclear of allocation concealment and high risk of other bias in Mi 2016 and Wang 2017, only a small number of participant were included for this outcome, and the corresponding author was one of the inventors of the RIC devices used in Mi 2016 and Wang 2017.

4 Serious inconsistency. We detected high level of the heterogeneity for the cognitive impairment.

5 Downgrade two levels by very serious risk of bias, serious inconsistency and serious indirectness. High risk of bias resulted from the unclear of allocation concealment,

the unblinded treatment protocol to the participants, and large number of participants lost to follow up during the study period in Hougaard 2014; and the small number of

participants in England 2016.

6 Serious indirectness. The infarct volume was used as a surrogate endpoint to assess stroke severity in Hougaard 2014. 


\section{B A C K G R O U N D}

\section{Description of the condition}

Stroke is the leading cause of adult disability globally, and its disease burden continues to increase (Feigin 2015; Roth 2015). Ischaemic stroke, which is due to blockage of a brain blood vessel, accounts for nearly $90 \%$ of all strokes; and nearly a quarter of individuals who have an ischaemic stroke will have recurrent strokes (Writing Group 2016). In 2013, more than 70\% of stroke survivors and more than $50 \%$ of deaths from stroke were attributed to ischaemic stroke (Feigin 2015).

Fortunately, most ischaemic strokes are preventable. Prevention and screening of stroke not only reduces the incidence of ischaemic stroke and subsequent disability but may have beneficial effects on cognitive decline and other neuropsychological diseases. Key strategies for preventing ischaemic stroke include control of risk factors, anti-clotting therapy, intervention for vascular obstruction and lifestyle change (Steiger 2016). Although these strategies play important roles in clinical practice, some are not cost effective and others may result in severe adverse events.

Timely intervention to open the occluded artery and return blood flow to salvageable cerebral tissue via clot-treating therapies (including systemic and local administration of drugs to break up clots and use of various intra-arterial minimally-invasive devices to manually remove clots) are the most effective methods for salvaging brain tissue that had interrupted blood flow but is not already dead. The exact window of time for maximally effective treatment is unknown, but strong evidence shows that systemic administration of a drug to break up clots is only beneficial within 4.5 hours of symptom onset, while minimally-invasive management with second-generation mechanical clot-removal devices is beneficial for patients with specific type of stroke within 24 hours of symptom onset (Powers 2018; Nogueira 2018). Unfortunately, these treatments are expensive and are available only at select comprehensive stroke centres. Furthermore, injury to surviving brain tissue after reestablishment of blood flow (a phenomenon known as ischaemia/reperfusion injury) is a major source of morbidity and mortality (Bai 2015). These disadvantages severely influence the prognosis of ischaemic stroke worldwide, especially in countries with low to medium levels of resources, such as China.

\section{Description of the intervention}

Ischaemic conditioning is a strategy by which one or more cycles of a brief period of interruption of blood flow to an organ or tissue followed by resumption of flow confer protection against subsequent, more severe ischaemic events (Hausenloy 2016). This far-reaching phenomenon was first demonstrated by Murry and colleagues (Murry 1986). Since that time, ischaemic conditioning has been widely studied. However, a major disadvantage of attempting to condition a specific organ is that it requires application of an intervention directly to the target, which is not always feasible in clinical practice. A strategy by which the protective conditioning cycles are applied to an organ or tissue remote from the target organs may offer a far more attractive clinical application. Przyklenk and colleagues introduced a new method of carrying out ischaemic conditioning, in which the conditioning cycles of ischaemia were applied to a separate region (Przyklenk 1993). Later on, this strategy was named 'remote ischaemic conditioning' (RIC). Investigators have evaluated many tissues and organs such as kidney, liver, limbs, and the mesenteric artery as remote conditioning sites in animals. RIC performed on limbs, generally with blood pressure cuffs inflated to a pressure that blocks blood flow in one or both directions, is now considered the most convenient and feasible method that can be used in clinical settings (Hausenloy 2016; Pan 2016). RIC can be divided into three types on the basis of initiation of conditioning: preconditioning (RIC applied before ischaemia), perconditioning (RIC applied after ischaemia onset and before reperfusion), and postconditioning (RIC applied during reperfusion) (Pan 2016).

Many research studies have shown RIC to be a well tolerated and effective strategy for protecting the brain from ischaemia or reperfusion injury (Hahn 2011; Hoda 2012; Hougaard 2014; Pan 2016; Ren 2015). It has been determined that RIC could increase brain tolerance to injury caused by ischaemia, reduce the risk of cerebral infarction, improve blood flow to the brain, and promote the formation of alternately-routed blood vessels to the brain (Hougaard 2014; Meng 2012; Pan 2016). Several randomised controlled trials (RCTs) have reported that RIC might confer neuroprotection against damage from acute ischaemic stroke and chronic cerebral ischaemia (Hougaard 2014; Meng 2012; Meng 2015). Although evidence suggests potential benefit of RIC for people with ischaemic stroke, many questions remain unanswered. The underlying protective mechanisms of RIC still need to be elucidated, and the optimal duration and number of ischaemia/ reperfusion cycles have not been established. Additionally, the potential for complications related to pressure cuffs is regarded as low, and other adverse effects associated with RIC remain unknown. Finally, criteria are needed that can be used to identify ideal candidate people for RIC.

\section{How the intervention might work}

Characterisation of the mechanism by which RIC provides protection for the brain is currently incomplete, and most evidence has been provided by studies on cardiac preconditioning and postconditioning. Underlying mechanisms include two windows of protection: the early phase (action immediately after a stimulus that might play a role through affecting critical proteins) and the delayed phase (action days or weeks after a stimulus that might play a role in gene regulation and new protein synthesis) (Hahn 2011). Research findings indicate that protective signals may be transmitted from the conditioned organ to the brain through three routes: humoral, neuronal, and immunological pathways (Weber 2010).

Substances or humoral factors generated by the conditioned site may be circulated to the brain through the blood upon reestablishment of blood flow of the conditioned site and may exert protective effects through signal pathways. A neuronal pathway at conditioned sites might be stimulated by local production of factors such as adenosine, bradykinin or other neuropeptides, which then stimulate nerve signals to the brain, thus inducing neuroprotection. RIC may derive its protective effects via alteration of gene and small molecule expression within the immune system, which then activates immune cells and alters inflammatory responses (Lim 2012; Pan 2016). Therefore, although the three protective signal pathways are distinct, both neuronal and immunological pathways are linked to the humoral pathway. 


\section{Why it is important to do this review}

RIC appears to be a well tolerated, cost-effective, easily implemented strategy that has the potential to provide specialised care for any user (e.g. patients, caregivers, physicians) in any circumstance (e.g. home, ambulance, hospital). Previous Cochrane review authors have evaluated RIC in vascular and endovascular surgical procedures (Desai 2011), and RIC has also be evaluated in coronary artery bypass grafting and kidney ischaemia-reperfusion injury in theCochrane Database of Systematic Reviews (Menting 2017; Benstoem 2017). RIC is developing as a neuroprotective strategy for ischaemic stroke prevention and treatment, and its implementation in clinical settings is increasing in many parts of the world. Several clinical trials have shown that RIC might reduce stroke recurrence and improve stroke outcomes, and that it is well tolerated without associated severe adverse events (Hougaard 2014; Meng 2012; Meng 2015). However, existing evidence is insufficient to demonstrate the efficacy and safety of RIC.

\section{OB JECTIVES}

To assess the benefits and harms of RIC for preventing ischaemic stroke and for treating people with ischaemic stroke and those at risk for ischaemic stroke.

\section{METHODS}

\section{Criteria for considering studies for this review}

\section{Types of studies}

We included all identified randomised controlled trials (RCTs) comparing remote ischaemic conditioning (RIC) versus non-RIC (sham RIC or standard medical management alone) in people with ischaemic cerebrovascular diseases. We excluded uncontrolled trials as well as quasi-RCTs where allocation to treatment or control was not concealed (e.g. allocation by alteration, open random number list, date of birth, day of the week, or hospital number).

\section{Types of participants}

We included participants with any of the following: (1) acute ischaemic stroke diagnosed on the basis of the a combination of clinical examination findings and diagnostic test results or according to definitions for acute ischaemic stroke used by researchers to enrol patients in their RCTs; (2) chronic cerebral ischaemia (i.e.14 days after symptom onset) or gradual-onset cerebral ischaemia (e.g. small vessel disease); (3) a definitive diagnosis of intracranial or extracranial cerebrovascular moderate and severe stenosis (i.e. $>50 \%$ ) or occlusion confirmed by computed tomography angiography (CTA), magnetic resonance angiography (MRA) or digital subtraction angiography (DSA). We excluded studies of participants undergoing cardiovascular intervention therapies, coronary artery bypass grafting, any other type of cardiac surgery, and people undergoing other surgeries, as the complication risk of ischaemic stroke was low in these patients.

\section{Types of interventions}

The RIC protocol consisted of remote ischaemic preconditioning, remote ischaemic perconditioning and remote ischaemic postconditioning, which were the same procedure but divided based on the order of its initiation and the occurrence of ischaemia. A remote stimulus was applied to any organ (e.g. upper and lower limbs). This stimulus consisted of one or more cycles of brief ischaemia and reperfusion of varying duration. The control condition consisted of no intervention or standard medical management and shamRIC,which required application of a blood pressure cuff, tourniquet or other occlusive device without full interruption of blood flow.

\section{Types of outcome measures}

\section{Primary outcomes}

- Ischaemic stroke or recurrent ischaemic stroke at completion of follow-up

- Death or dependency (e.g. Barthel Index (BI) score $\leq 60$, modified Rankin Scale (mRS) grade 3 to 6 , defined by the trial author) at completion of follow-up

\section{Secondary outcomes}

- Stroke severity as measured by infarct volume, the National Institutes of Health Stroke Scale (NIHSS), the Canadian Neurological Scale (CNS), the Scandinavian Stroke Scale, the European Stroke Scale, the modified Rankin Scale (mRS) or other scales reported by trial authors

- Improvement in neurological impairment (e.g. NIHSS, mRS, CNS, other scales defined by trial authors) at completion of follow-up

- Improvement in psychological and cognitive impairment (e.g. Mini-Mental State Examination (MMSE), Montreal Cognitive Assessment (MoCA), Wechsler Memory Scale (WMS), Wechsler Memory Scale-Revised (WMS-R), Luria-Nebraska Neuropsychological Battery (LNNB) Memory Scale, other scales defined by trial authors) at completion of follow-up

- Cardiovascular events (e.g. acute myocardial infarction, angina)

- Haemorrhagic stroke (e.g. intracerebral haemorrhage, subarachnoid haemorrhage)

- Adverse events associated with RIC treatment (e.g. soft tissue injury, venous thrombosis, artery dissection, events reported by trial authors)

\section{Search methods for identification of studies}

See the 'Specialised register' section in the Cochrane Stroke Group module. We attempted to identify all relevant RCTs without restriction on language or publication status, and arranged translation of relevant papers where necessary.

\section{Electronic searches}

We searched the Cochrane Stroke Group Trials Register (16 January 2018), and the following electronic bibliographic databases.

- Cochrane Central Register of Controlled Trials (CENTRAL; 2017, Issue 12) in the Cochrane Library (searched 16 January 2018) (Appendix 1)

- MEDLINE Ovid (from 1946 to 16 January 2018) (Appendix 2)

- Embase Ovid (from 1974 to 16 January 2018) (Appendix 3)

- Science Citation Index Expanded (SCI-EXPANDED) (Web of Science; 1950 to 10 January 2018) (Appendix 4)

- Conference Proceedings Citation Index- Science (CPCI-S) (Web of Science; 1950 to 10 January 2018) (Appendix 4)

- China Biological Medicine Database (CBM; www.sinomed.ac.cn/) (from 1978 to 10 January 2018) (Appendix 5) 
- China Doctoral Dissertations Full-text Database (CDFD) (CNKI from 1984 to 10 January 2018) (Appendix 6)

- China Academic Journal Network Publishing Database (CAJD) (CNKI; from 1915 to 10 January 2018) (Appendix 7)

We developed the MEDLINE search strategy with the help of the Cochrane Stroke Group Information Specialist and adapted it for the other databases (Appendix 2).

We also conducted a search of the following research registers to identify ongoing matched trials (10 January 2018).

- World Health Organization (WHO) International Clinical Trials Registry Platform (www.who.int/trialsearch) (Appendix 8)

- ClinicalTrials.gov (www.clinicaltrials.gov/) (Appendix 9)

- Stroke Trials Registry (www.strokecenter.org/trials/) (Appendix 10)

- ISRCTN Registry (www.isrctn.com) (Appendix 11)

\section{Searching other resources}

In an effort to identify other published, unpublished, and ongoing studies, we searched reference lists of relevant trials, and relevant journals (Brain Circulation and Conditioning Medicine) and conference proceedings (European Stroke Conference, International Stroke Conference, and Neuroscience Conferences). We also searched for and contacted trialists and researchers in the field where necessary.

\section{Data collection and analysis}

\section{Selection of studies}

Two review authors (WBZ and JZ) independently screened titles and abstracts of records identified through literature search activities and excluded those that were obviously irrelevant and duplicated. We retrieved the full text of remaining articles, and the same two review authors (WBZ and JZ) independently screened full-text articles to identify studies for inclusion or exclusion on the basis of our predetermined Criteria for considering studies for this review. The two authors resolved any disagreements through discussion or by consulting a third author (RM).

\section{Data extraction and management}

Two review authors (WBZ and JZ) independently extracted and recorded study details on a data extraction form. We extracted the following data: methods, characteristics of participants, interventions, treatment duration, primary and secondary outcomes and time points reported. The same two authors crosschecked all extracted data and resolved any differences in the extracted data by referring to the original articles and through discussion. We extracted data to allow an intention-to-treat (ITT) analysis (including all participants in the groups to which they were originally randomly allocated).

For continuous outcomes, we used means and standard deviations for each group. If median and interquartile ranges were used in the original article, we translated them to means and standard deviations (Wan 2014). For binary outcomes, we extracted the number of participants with the event in each group.

\section{Assessment of risk of bias in included studies}

Two review authors (WBZ and JZ) independently assessed risk of bias for each study using the criteria outlined in theCochrane Handbook for Systematic Reviews of Interventions (Higgins 2011). We resolved disagreements by discussion or by consultation with a third review author (RM) where necessary. We assessed risk of bias according to the following domains: selection bias; performance bias; detection bias; attrition bias; reporting bias and other possible bias.

For each domain, we categorised each study as presenting 'low risk', 'high risk', or 'unclear risk' of bias and provided information from the study report, together with a justification for our judgement, in the 'Risk of bias' tables.

\section{Measures of treatment effect}

We calculated risk ratios (RRs) with 95\% confidence intervals (Cls) for dichotomous data (e.g., ischaemic stroke, recurrent ischaemic stroke, death, dependency, haemorrhagic stroke, cardiovascular events). For continuous data (e.g. scores from scales), we used mean differences (MDs) with $95 \% \mathrm{Cls}$ for data measured in the same way between trials, and standardised mean differences (SMDs) with $95 \% \mathrm{Cls}$ to combine data when different scales were used for measurement. We narratively described skewed data reported as medians and interquartile ranges.

\section{Unit of analysis issues}

In most studies, the unit of analysis was the individual participant and a single measurement for each outcome was collected and analysed. However, for cross-over trials, the unit of analysis included only the first phase in the analysis. For cluster-randomised trials, the unit of analysis was the cluster.

\section{Dealing with missing data}

We carried out analyses according to the ITT principle: we used the number of initially randomised individuals as the denominator. If data were missing, we contacted the corresponding author or co-authors through the address or email given in the publication. When this was not possible, we calculated best- and worst-case scenarios for dichotomous data: the best-case scenario would mean participants with missing data were alive or achieved dependence or did not experience stroke, and the worst-case scenario would mean that participants with missing data were dead or experienced stroke or did not achieve dependence.

\section{Assessment of heterogeneity}

We assessed heterogeneity between study results using the 12 statistic. We defined a value greater than $50 \%$ as indicating substantial heterogeneity.

\section{Assessment of reporting biases}

We had intended to use funnel plots to assess the potential existence of reporting bias if more than 10 studies were included in this review. However, because we only seven included studies, we assessed reporting bias qualitatively on the basis of the study characteristics. 


\section{Data synthesis}

We conducted a meta-analysis using Review Manager 5 (Review Manager 2014). We used RR as a measure of effect for binary outcomes and MD or SMD as a measure of effect for continuous outcomes. We used a random-effects model to better estimate the effect size of different studies, regardless of whether heterogeneity was significant.

\section{GRADE and 'Summary of findings' table}

We summarised the evidence by creating 'Summary of findings' tables using GRADEproGDT (https://gradepro.org/), in which we imported data from Review Manager 5. We used the GRADE approach to assess the quality of evidence as high, moderate, low, or very low, based on the following five considerations: study limitations, imprecision, consistency of effect, indirectness and publication bias (Higgins 2011). The table presents results and our assessments of the quality of evidence for the main outcomes.

\section{Subgroup analysis and investigation of heterogeneity}

We had intended to performed subgroup analysis to investigate heterogeneity by analysing differences in the number of RIC cycles and their length, the position of RIC (upper versus lower limb, unilateral versus bilateral), and length of RIC treatment (fractionated versus continuous). However, in this review only seven trials were eligible for analysis: three studies for the analysis of RIC for preventing ischaemic stroke, and another four studies for the analysis of RIC for treating ischaemic stroke. Given the limited number of included studies, there were not enough data for subgroup analysis.

\section{Sensitivity analysis}

We had intended to perform sensitivity analyses if the results were substantially heterogeneous, by 1 ) excluding studies with inadequate allocation concealment; 2 ) excluding studies that did not use blinding; 3) excluding studies in which loss to follow-up was not reported or was greater than $10 \%$; 4 ) excluding unpublished studies; 5) excluding studies that did not use sham as a comparison; and 6) excluding long studies, large studies or those using imputed data to determine their influence on study results. However, we did not perform any sensitivity analyses because of the limited number of included studies, and no substantial heterogeneity was found in this review.

\section{RE S U L T S}

\section{Description of studies}

A detailed description of all studies can be found in Characteristics of included studies, Characteristics of excluded studies, and Characteristics of ongoing studies.

\section{Results of the search}

We identified a total of 8765 records from database searching in January 2018. After removing 1668 duplicates, 7097 records remained, which we screened and excluded 7066 records. We retrieved 31 full-text articles and abstracts and assessed them for eligibility. Finally, we identified seven trials and assessed them for eligibility as per protocol with refined inclusion criteria. We excluded 16 studies with reasons (see Characteristics of excluded studies), and identified eight ongoing studies. We present details of the seven included trials in Characteristics of included studies. For an illustration of these results, please see the study flow diagram (Figure 1). 
Figure 1. Study flow diagram.

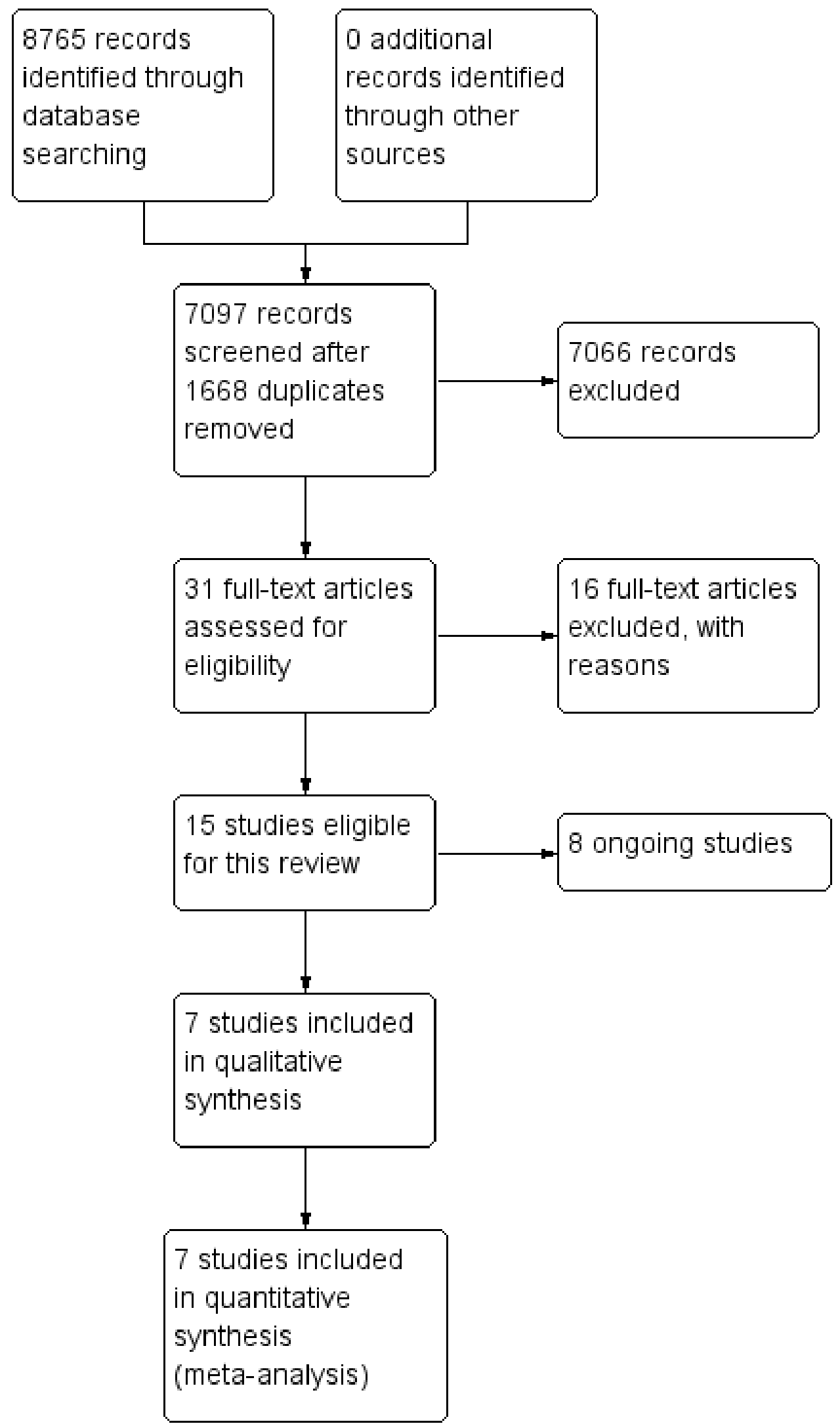




\section{Included studies}

Seven trials met our inclusion criteria. The first trial was a single-centre trial conducted in the UK (England 2016). The trial compared remote ischaemic conditioning (RIC) with sham RIC in 26 participants (13 in each group) with acute ischaemic stroke. RIC and sham RIC were started within 24 hours of stroke onset and performed manually using a standard upper arm blood pressure cuff in the nonparetic arm. RIC consisted of four cycles of five minutes' inflation ( $20 \mathrm{mmHg}$ above systolic blood pressure) and five minutes' deflation; and sham RIC consisted of four cycles of five minutes' inflation ( $30 \mathrm{mmHg}$ ) and five minutes' deflation. The primary outcome was tolerability and feasibility of RIC after acute ischaemic stroke, and the duration of follow-up was 90 days.

The second trial was a single-centre trial conducted in Denmark (Hougaard 2014). The trial compared intravenous thrombolysis plus RIC with intravenous thrombolysis alone in 285 participants with acute ischaemic stroke. Because of the imbalanced randomisation and large number of participants excluded from the analysis after randomisation, there were unbalanced numbers of participants between the two groups (160 in the intervention group, and 125 in the control group). RIC was started in the ambulance and induced by four inflations of a standard upper limb blood pressure cuff to either $200 \mathrm{mmHg}$ or $25 \mathrm{mmHg}$ above the systolic blood pressure, each lasting five minutes and separated by five minutes of cuff deflation. The primary outcome was the penumbra salvage, and the duration of follow-up was 90 days.

The third trial was conducted in two centres in China (Meng 2012). The trial compared standard medical treatment plus RIC and standard medical treatment alone in 103 participants (51 in the intervention group and 52 in the control group) with symptomatic intracranial artery stenosis. RIC, consisting of five cycles of bilateral arms ischaemia (induced by inflating tourniquets to $200 \mathrm{mmHg}$ ) for five minutes followed by reperfusion for another five minutes, was performed twice daily for a total of 300 consecutive days. Standard medical treatment including antiplatelet agents, lipid control agents, and antidiabetic agents (if necessary). The primary outcome was time point of the first stroke recurrence event, and the duration of follow up was 300 days.

The fourth trial was a single-centre trial conducted in China (Meng 2015). The trial compared RIC and sham RIC in 79 octogenarian and nonagenarian participants (40 in the intervention group and 39 in the control group) with symptomatic intracerebral artery stenosis. RIC consisted of five cycles of simultaneous bilateral arms ischaemia (induced by inflating tourniquets to $200 \mathrm{mmHg}$ ) for five minutes followed by reperfusion for another five minutes. Sham RIC consisted of five cycles of bilateral arms ischaemia (induced by inflating tourniquets to $30 \mathrm{mmHg}$ ) for five minutes, followed by reperfusion for another five minutes. Both RIC and sham RIC were performed for 180 consecutive days. Medical management included either clopidogrel $75 \mathrm{mg} /$ day in combination with atorvastatin $20 \mathrm{mg} /$ day or aspirin $100 \mathrm{mg} /$ day and clopidogrel 75 $\mathrm{mg} /$ day in combination with atorvastatin $20 \mathrm{mg} /$ day. The duration of follow-up was 180 days.

The fifth trial was a single-centre trial conducted in China (Mi 2016). The trial compared RIC and sham RIC in 17 participants (nine in the intervention group and eight in the control group) with cerebral small vessel disease. Both RIC and sham RIC were achieved using an automated cuff inflator placed on bilateral arms that were inflated to $200 \mathrm{mmHg}$ and $50 \mathrm{mmHg}$ respectively. Both RIC and sham RIC consisted of five cycles of five minutes' ischaemia follow by five minutes' reperfusion. All participants were required to receive RIC or sham RIC treatment twice daily for one year. The primary outcome was changes in brain lesions, evaluated by the number of lacunar infarctions and white matter lesions volume, and the duration of follow-up was one year.

The sixth trial was a single-centre trial conducted in China (Wang 2017). The trial compared RIC and sham RIC in 36 participants (18 in the intervention group and 18 in the control group) with cerebral small vessel disease-related mild cognitive impairment. Both RIC and sham RIC were achieved using an automated cuff inflator placed on bilateral arms that were inflated to $200 \mathrm{mmHg}$ and 50 $\mathrm{mmHg}$ respectively. Both RIC and sham RIC consisted of five cycles of five minutes' ischaemia follow by five minutes' reperfusion. All participants were required to receive RIC or sham RIC treatment twice daily for one year. The primary outcome was the change of brain lesions, and the follow-up duration was one year.

The seventh trial was a single-centre trial conducted in China (Zhao 2017). The trial compared RIC plus standard medical treatment, sham RIC plus standard medical treatment and standard medical treatment alone in 189 participants (63 in each group) with severe carotid artery stenosis. RIC and sham RIC consisted of five cycles of bilateral arms ischaemia for five minutes, followed by reperfusion for another five minutes, and they were performed twice daily for two weeks before carotid stenting. Both RIC and sham RIC procedures were performed by using an electric autocontrol device with cuffs that inflated to a pressure of $200 \mathrm{mmHg}$ and $60 \mathrm{mmHg}$ respectively during the ischaemic period. The primary outcomes were the presence of one or more new brain lesions on diffusion weighted imaging (DWI) within 48 hours after carotid artery stenting, and the incidence of clinical events (i.e. ischaemic stroke, transient ischaemic attach (TIA), acute myocardial infarction, haemorrhagic stroke, hyperperfusion syndrome, and death) within six months after stenting. The duration of follow-up was six months.

\section{Excluded studies}

We excluded 16 studies from this review. The Characteristics of excluded studies table provides summary details.

We excluded seven studies because the participants underwent percutaneous coronary intervention or cardiac surgery (Cho 2017; Coverdale 2017; Davies 2013; Hoole 2009; Hudetz 2014; Sloth 2014; Zhong 2013). We excluded one study because the included participants underwent transfemoral transcatheter aortic valve implantation (Kahlert 2017). One study randomised participants treated with major vascular surgery, including carotid endarterectomy; we excluded it because we did not obtain the data on participants treated with carotid endarterectomy after emailing the author (Healy 2015). We excluded four studies because they did not assess ischaemic stroke in their outcome assessment (Jing 2011; Meng 2017; Sales 2017; Walsh 2010). Another study was not a randomised controlled trial (Joseph 2015), and another study has passed the completion date but the status has not been verified after more than two years, and no paper or results have been published to date (NCT01672515). We excluded one study because the comparison was between healthy people and patients (Hyngstrom 2016). 


\section{Risk of bias in included studies}

The 'Risk of bias' assessments for all included studies are summarised in Figure 2 and Figure 3.

Figure 2. 'Risk of bias' graph: review authors' judgements about each risk of bias item presented as percentages across all included studies.

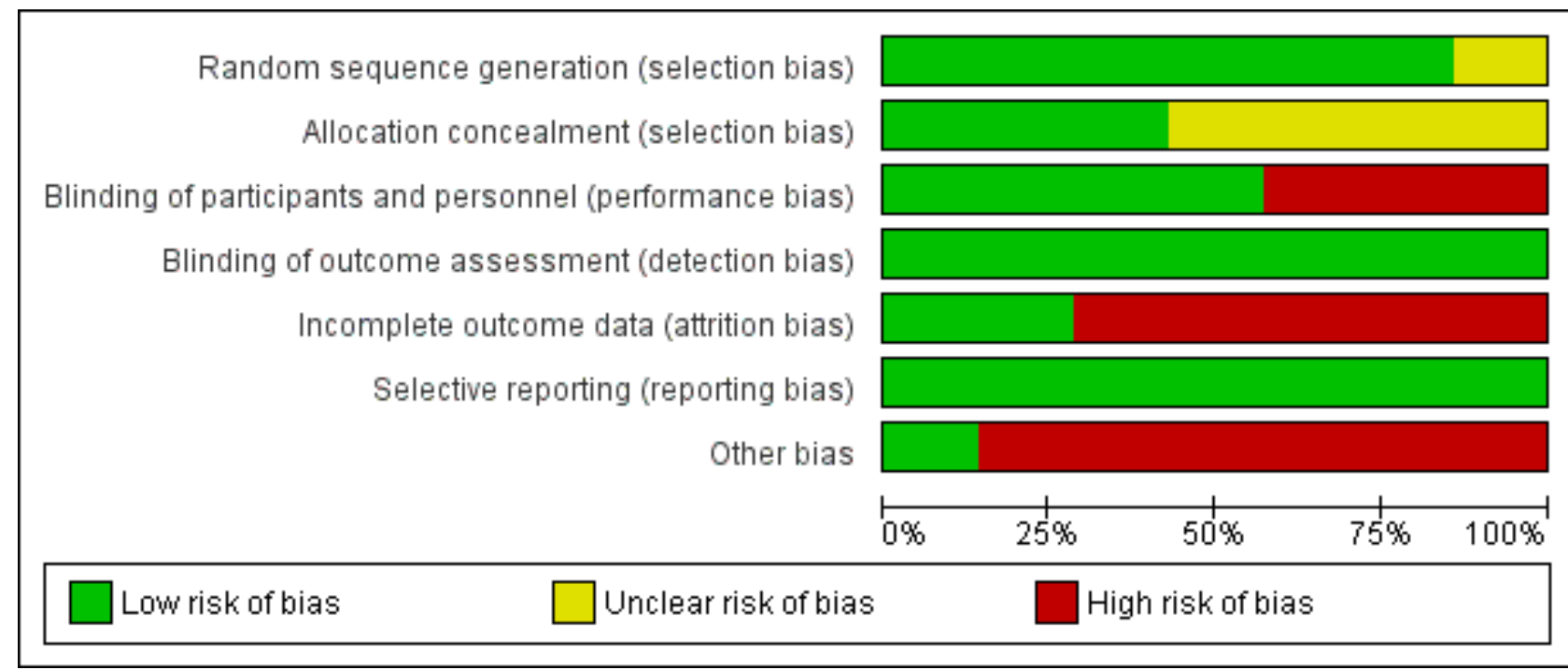


Figure 3. 'Risk of bias' summary: review authors' judgements about each risk of bias item for each included study.

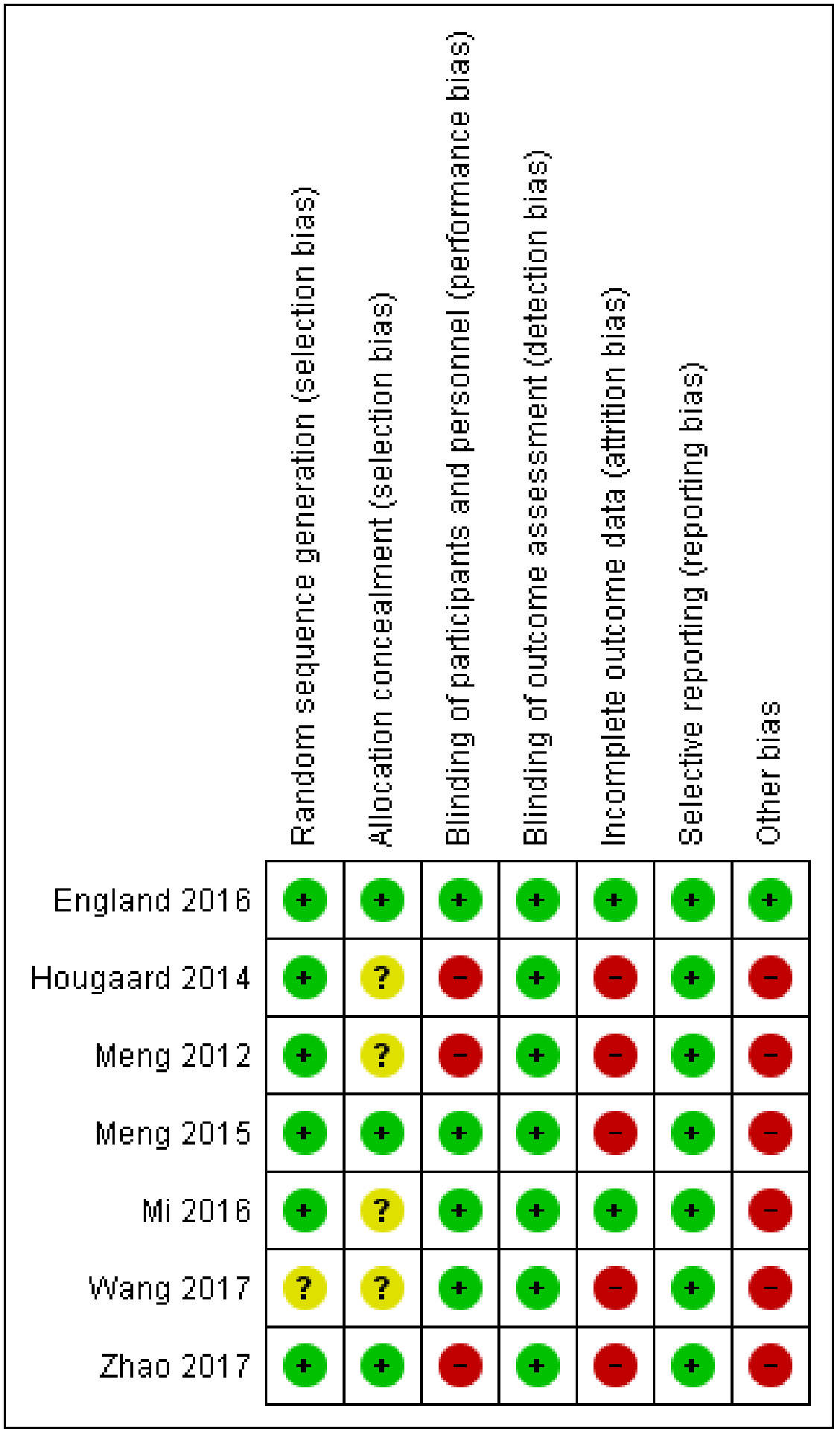

\section{Allocation}

The method of randomisation was described and considered adequate with low risk of bias in six included studies (England 2016; Hougaard 2014; Meng 2012; Meng 2015; Mi 2016; Zhao 2017). Four studies reported that the random sequence was generated by using computer-generated random number sequence (Meng 2012; Meng 2015; Mi 2016; Zhao 2017). One study reported that the randomisation was performed by drawing from a large number of sealed opaque envelopes (Hougaard 2014). One study reported that the randomisation was generated by minimisation (England 2016). Another study only reported that randomisation was performed by an independent statistician; the details were not reported, therefore we judged the risk of bias as unclear (Wang 2017). 
Allocation concealment was adequate with low risk of bias in three studies, one of which used a web-based randomisation method (England 2016), and two of which used sequentially numbered, opaque, sealed envelopes (Meng 2015; Zhao 2017). Allocation concealment was unclear for three trials which only reported that the assignments were sealed, but not whether the assignment was sequentially numbered (Hougaard 2014; Mi 2016; Wang 2017). One study did not report the details of allocation (Meng 2012).

\section{Blinding}

Because of the nature of RIC treatment, if no sham RIC treatment was used, it was impossible to blind the participants. Therefore, we judged two studies as being at high risk of bias (Hougaard 2014; Meng 2012), and four as being at low risk of bias (Meng 2015; England 2016; Mi 2016; Wang 2017). One study was comprised of three groups (RIC group, sham RIC group, and control group); although sham RIC was included in the study, it was also impossible to blind the participants (Zhao 2017). Therefore, we also judged this trial as having high risk of performance bias.

One study was an outcome-blinded, placebo-controlled trial (England 2016). All other studies reported that the outcome assessors were blinded to the treatment protocol (Hougaard 2014; Meng 2012; Meng 2015; Mi 2016; Wang 2017; Zhao 2017). We therefore judged all the trials as having low risk of detection bias.

\section{Incomplete outcome data}

We judged two studies as having low risk of attrition bias as there were no participants lost to follow-up during the study period (England 2016; Mi 2016). We judged three trials as having high risk of attrition bias because of the following losses to follow-up: 35 out of 103 (34.0\%) participants (Meng 2012); 21 out of 79 (26.6\%) participants (Meng 2015); and 6 out of 36 (16.7\%) participants (Wang 2017). We judged the two remaining studies as having high risk of attrition bias because 158 out of 443 (35.7\%) participants were excluded in the as-treated analyses (Hougaard 2014); and 27 out of $189(14.3 \%)$ participants were lost to the analyses of imaging and 42 out of $189(22.2 \%)$ participants were lost to the analyses of clinical events (Zhao 2017).

\section{Selective reporting}

All included studies registered their study protocols in one of the clinical trial registration websites, and all predefined study outcomes were published. Therefore, we judged all included studies as low risk of reporting bias (England 2016; Hougaard 2014; Meng 2012; Meng 2015; Mi 2016; Wang 2017; Zhao 2017).

\section{Other potential sources of bias}

We judged five trials performed in the same centre in China to be high risk of other bias because these trials had the same principle investigator, who was one of the inventors of the electric autocontrol RIC device used in these studies (Meng 2012; Meng 2015; Mi 2016; Wang 2017; Zhao 2017). We judged one other study to be at high risk of other bias because two authors were shareholders in the company where the devices used in the study were made (Hougaard 2014). We judged the remaining study to be at low risk of other potential bias as we identified no other bias (England 2016).

\section{Effects of interventions}

See: Summary of findings for the main comparison RIC versus non-RIC for preventing ischaemic stroke; Summary of findings 2 $\mathrm{RIC}$ versus non-RIC treating ischaemic stroke

See: Summary of findings for the main comparison and Summary of findings 2.

Three studies focused on remote ischaemic conditioning (RIC) for preventing ischaemic stroke (Meng 2012; Meng 2015; Zhao 2017), while the other four studies focused on RIC for treating ischaemic stroke (England 2016; Hougaard 2014; Mi 2016; Wang 2017). The outcomes used in these two categories of studies were different. Therefore, we analysed RIC for preventing and treating ischaemic stroke separately. Although multiple relative risks were used for outcomes analyses, caution should be taken when using these results as the presented data were extracted from only one or two studies with small sample sizes and low quality of evidence.

\section{RIC versus non-RIC for preventing ischaemic stroke}

Three studies focused on RIC for preventing ischaemic stroke (Meng 2015; Meng 2012; Zhao 2017). The total number of participants was 371.

\section{Primary outcomes}

\subsection{Ischaemic stroke or recurrent ischaemic stroke at completion of follow-up}

Ischaemic stroke was reported in Zhao 2017 and recurrent ischaemic stroke was reported in Meng 2012 and Meng 2015.

\subsubsection{Ischaemic stroke}

One study (Zhao 2017), involving 189 participants (50.9\% of the total number of participants included in this part of review) presented data on ischaemic stroke. There was no significant difference in the incidence of ischaemic stroke between those allocated to RIC versus those allocated to non-RIC (risk ratio (RR) $0.22,95 \%$ confidence interval $(\mathrm{Cl}) 0.01$ to $4.03 ; \mathrm{P}=0.31$ ) (Analysis 1.1).

\subsubsection{Recurrent ischaemic stroke}

Two studies (Meng 2012; Meng 2015), involving 182 participants (49.1\% of the total number of participants included in this part of review), presented data on recurrent ischaemic stroke. The incidence of recurrent ischaemic stroke was significantly reduced by RIC treatment (RR $0.32,95 \% \mathrm{Cl} 0.12$ to $0.83 ; \mathrm{P}=0.02$ ) (Analysis 1.1).

\subsection{Death or dependency at completion of follow-up}

Death was not reported in Meng 2015. The other two studies (Meng 2012; Zhao 2017) reported that no participant died during the study period, so analysis was not applicable to the outcome of death. None of the trials (Meng 2012; Meng 2015; Zhao 2017) reported any forms of dependency that enabled us to include them for analysis.

\section{Secondary outcomes}

\subsection{Stroke severity}

This outcome was assess by infarct volume in Zhao 2017, which involving 189 participants $(50.9 \%$ of the total number of participants included in this part of review). Infarct volume 
was calculated using magnetic resonance imaging (MRI) scans. In participants with multiple lesions, the total infarct volume was added up. The infarct volume was significantly smaller in participant treated with RIC compared with those not treated with RIC (MD - $0.17 \mathrm{~mL}, 95 \% \mathrm{Cl}-0.23$ to $-0.11 ; \mathrm{P}<0.01 ; \mathrm{I}^{2}=3 \%$ ) (Analysis 1.3).

\subsection{Improvement in neurological impairment at completion of follow-up}

None of the three trials reported this outcome in a form that enabled us to include them for this analysis.

\subsection{Improvement in phycological and cognitive impairment at completion of follow-up}

None of the three trials reported this outcome in a form that enabled us to include them for this analysis.

\subsection{Cardiovascular events}

This outcome was not reported in Meng 2015. The other two studies (Meng 2012; Zhao 2017) reported that no participant experienced cardiovascular events during the study period, so analysis was not applicable to this outcome.

\subsection{Haemorrhagic stroke}

None of the three trials reported this outcome in a form that enabled us to include them for this analysis.

\subsection{Adverse events associated with RIC treatment}

All studies (371 participants: $100 \%$ of the total number of participants included in this part of review) reported data on adverse events associated with RIC treatment. One study reported that no participant experienced any adverse events associated with RIC treatment (Meng 2012). One study reported that 6/63 participants experienced arm skin petechiae from repeated pressure cuff applications (Zhao 2017), and the other study reported that sporadic petechiae in 3/40 participants were observed (Meng 2015). The adverse events associated with RIC treatment were significantly higher in participants treated with RIC (RR $10.91 ; 95 \% \mathrm{Cl} 2.01$ to 59.28; $P=0.006 ; \mathrm{I}^{2}=0 \%$ ). No participant experienced severe adverse events associated with RIC treatment.

\section{RIC versus non-RIC for treating ischaemic stroke}

Four studies focused on RIC for treating ischaemic stroke (England 2016; Hougaard 2014; Mi 2016; Wang 2017). The total number of participants was 364 .

\section{Primary outcomes}

\subsection{Ischaemic stroke or recurrent ischaemic stroke at completion of follow-up}

None of the four trials reported these outcomes in any forms that enabled us to include them for this analysis.

\subsection{Death or dependency at completion of follow-up}

Both death and dependency were was reported by one study including 285 participants $(78.3 \%$ of the total number of participants included in this part of review) (Hougaard 2014). Dependency, assessed by the mean and median of the modified Ranks Scale score and the Barthel Index, was reported in one study
(England 2016); we tried to contact the study authors to get the incidence of dependency, but failed to receive any response. Two other studies did not report any of the outcomes (Mi 2016; Wang 2017). Therefore we only included Hougaard 2014 in this analysis. The rate of death or dependency was significantly increased by RIC treatment compared with non-RIC treatment (RR 2.34; 95\% 1.19 to 4.61; $P=0.01$ ) (Analysis 2.2).

\section{Secondary outcomes}

\subsection{Stroke severity}

This outcome was assessed by the infarct volume in Hougaard 2014 and the NIHSS scores in England 2016.

\subsubsection{Final infarct volume}

This outcome was assessed by the final infarct volume in one study with 149 participants $(40.9 \%$ of the total number of participants included in this part of review) (Hougaard 2014). No significant difference was detected between participants allocated to RIC treatment and those allocated to non-RIC treatment (SMD $0.08 \mathrm{~mL}$; $95 \% \mathrm{Cl}-0.24$ to $0.41 ; \mathrm{P}=0.61$ ) (Analysis 2.3 ).

\subsubsection{NIHSS scores}

Scores on the NIHSS at three-month follow-up were reported in England 2016, which included 26 participants $(9.1 \%$ of the total number of participants included in this part of review). There was no significant difference between participants allocated to RIC treatment and those allocated to non-RIC treatment (SMD -0.73 points; $95 \% \mathrm{Cl}-1.53$ to $0.07 ; \mathrm{P}=0.07$ ) (Analysis 2.3 ).

\subsection{Improvement in neurological impairment at completion of follow-up}

None of the four trials reported this outcome in a form that enabled us to include them for this analysis.

\subsection{Improvement in phycological and cognitive impairment at completion of follow-up}

The phycological function was reported in England 2016 and the cognitive function was reported in three studies (England 2016; Mi 2016; Wang 2017).

\subsubsection{Psychological impairment}

Psychological impairment was only reported in England 2016, which included 26 participants $(9.1 \%$ of the total number of participants included in this part of review). The Zung Depression Score was used for the assessment. There was no significant difference between participants allocated to RIC treatment and those allocated to non-RIC treatment (SMD -0.37 points; $95 \% \mathrm{Cl}$ -1.15 to $0.41 ; \mathrm{P}=0.35$ ) (Analysis 2.5 ).

\subsubsection{Cognitive impairment}

Three studies reported results of the Mini-Mental State Examination (MMSE) (England 2016; Mi 2016; Wang 2017). Seventy-nine participants $(21.7 \%$ of the total number of participants included in this part of review) were included in this analysis. There was no significant difference in cognitive function between participants allocated to RIC treatment and those allocated to non-RIC treatment (SMD -0.26 points; $95 \% \mathrm{Cl}-0.72$ to $0.21 ; \mathrm{P}=0.28$ ) (Analysis 2.5). 


\subsection{Cardiovbascular events}

None of the trials reported this outcome in a form that enabled us to include them for this analysis.

\subsection{Haemorrhagic stroke}

None of the trials reported this outcome in a form that enabled us to include them for this analysis.

\subsection{Adverse events associated with RIC treatment}

None of the trials reported this outcome in a form that enabled us to include them for this analysis.

\section{Subgroup analyses}

Given the limited number of included studies, we did not perform subgroup analyses. If we include more studies in future updates of this review, we will explore differences in the results by creating subgroups according to the number of RIC cycles or their length, position of RIC treatment, and length of treatment.

\section{Sensitivity analyses}

We did not perform sensitivity analyses because of the limited number of included studies.

\section{Funnel plots}

We could not use funnel plots because only seven studies were included in this review, and three focused on RIC for preventing ischaemic stroke and the other four focused on RIC for treating ischaemic stroke.

\section{DISCUSSION}

In this Cochrane Review, we have assessed the benefits and harms of remote ischaemic conditioning (RIC) for either preventing or treating ischaemic stroke.

\section{Summary of main results}

\section{RIC for preventing ischaemic stroke}

Due to the low incidence of death and cardiovascular events in the study population, these analyses were not applicable. Two studies assessed effects of RIC in preventing recurrent ischaemic stroke in participants with symptomatic intracerebral artery stenosis (Meng 2012; Meng 2015), and one study assessed the effects of RIC in preventing ischaemic stroke after carotid stenting in participants with severe carotid stenosis (Zhao 2017). Based on the included studies, we found that RIC might prevent recurrent ischaemic stroke in people with symptomatic intracerebral artery stenosis, and reduce post-stenting infarct volumes in people undergoing carotid stenting.

Two studies reported adverse events associated with repeated RIC treatment, such as sporadic petechiae in local arm skin (Meng 2015; Zhao 2017). Although the adverse events associated with RIC treatment were significantly higher in participants treated with RIC, no severe adverse events (such as venous thrombosis, arterial dissection, or death) were reported.

\section{RIC for treating ischaemic stroke}

Two studies assessed the effects of RIC in treating acute ischaemic stroke (Hougaard 2014; England 2016), and another two studies assessed effects of RIC in treating cerebral small vessel disease (Mi 2016; Wang 2017). Based on the included studies, we found that RIC did not significantly reduce the final infarct volume in people with acute ischaemic stroke who received intravenous thrombolysis, but it might increase the death or dependency of these people. We also found that neurological impairment, assessed by the National Institutes of Health Stroke Scale (NIHSS) score, and psychological impairment were not significantly improved by RIC in this patient population. However, it should be noted that the sample size of one included trial was rather small $(n=26)$, and the number of participants lost to follow-up in another included study was rather high.

One study compared cognitive function between acute ischaemic stroke participants treated with RIC and non-RIC (England 2016), while two studies compared cognitive function between cerebral small vessel disease participants treated with RIC and non-RIC (Mi 2016; Wang 2017). There was no significant difference in cognitive function between the groups; however, it should be noted that the sample size of the three included trials was rather small $(n=79)$.

\section{Overall completeness and applicability of evidence}

In this review, we widened the protocol inclusion criteria to allow various RIC treatment protocols, and included all trials that recruited people with ischaemic cerebrovascular disease.

The three studies focused on preventing ischaemic stroke were carried out in China, and intracerebral artery atherosclerosis was common in this patient population. This may indicate that the results of RIC for preventing ischaemic stroke are likely to be only applicable to the Chinese population, and possibly to Asians, among whom there is a high prevalence of intracerebral artery atherosclerosis. The three included studies tested the same RIC treatment protocol (bilateral arms, five cycles of five minutes' ischaemia/reperfusion, twice daily) with different treatment duration (two weeks, 180 days, and 300 days). We found low-quality evidence that RIC could prevent recurrent ischaemic stroke in participants with symptomatic intracerebral artery stenosis, and reduce post-stenting new infarct volumes in participants undergoing carotid stenting. RIC treatment for preventing recurrent ischaemic stroke and ischaemic stroke should be reviewed in light of this evidence. Due to the low incidence of death and cardiovascular diseases, and the relatively small simple sizes of the included studies, the current review found no evidence that RIC could prevent death and cardiovascular diseases in people with ischaemic cerebrovascular disease. The three included studies reported RIC-specific adverse events, such as sporadic localised petechiae in arm skin, but none of them reported any severe adverse events related to RIC treatment.

Four studies focused on RIC for treating ischaemic stroke. One recruited participants with acute ischaemic stroke within 24 hours of symptom onset in the UK (England 2016), one recruited ischaemic stroke candidates considered for intravenous thrombolysis within 4.5 hours in Denmark (Hougaard 2014), one recruited participants with cerebral small vessel disease in China (Mi 2016), and one recruited participants with cerebral small vessel disease-related mild cognitive impairment in China (Wang 2017). This may mean that the results of this review are likely to be applicable to people from these countries. The included studies tested two treatment protocols (four cycles of unilateral arm and five cycles of bilateral arms, five minutes' ischaemia/reperfusion), 
and treatment duration of RIC varied from once to twice daily for 12 consecutive months. We found that RIC may increase death or dependency in people with acute ischaemic stroke who received intravenous thrombolysis, but we did not find any clear evidence that RIC had any effects on the final infarct volume, or psychological impairment in people with acute ischaemic stroke. Neither did we find any influence of RIC on the cognitive impairment in people with acute ischaemic stroke and cerebral small vessel disease. RIC for treating acute ischaemic stroke and cerebral small vessel disease should be reviewed in light of this evidence. Given the poor prognosis of acute ischaemic stroke, especially in those with large artery occlusion, further evidence relating to the use of RIC in this patient population or in combination with endovascular therapy or intravenous thrombolysis would be useful to better clarify the potential benefits and risks in this scenario.

\section{Quality of the evidence}

We assessed the quality of the evidence by using the GRADE approach (Guyatt 2008), and we presented the results in Summary of findings for the main comparison and Summary of findings 2 .

Four studies did not specify how allocation concealment was achieved, so we assessed them as having unclear risk of selection bias. Three studies were not blinded for participants, so we assessed them as having high risk of performance bias; and six studies had a high percentage of participants lost to follow-up, so we assessed them as having high risk of attrition bias. Overall, for each of the outcomes reported in our 'Summary of findings' tables, we graded the quality of evidence as low or very low.

From the three studies focused on RIC for preventing ischaemic stroke, there is low-quality evidence that RIC performed better than non-RIC in preventing recurrent ischaemic stroke in people with symptomatic intracerebral artery stenosis if treated for several consecutive months, and in reducing incidence of post-stenting new infarct volume in people undergoing carotid stenting if treated for two consecutive weeks before stenting. However, further research is likely to have a substantial impact on our confidence in the above estimations.

Four studies focused on RIC for treating ischaemic stroke. There is low-quality evidence that RIC might increase death or dependency in acute ischaemic stroke participants treated with intravenous thrombolysis. There is low-quality evidence that RIC performed no better or no worse than non-RIC in improving psychological impairment in people with acute ischaemic stroke if started within 24 hours of ictus, and there is low-quality evidence that RIC performed no better or no worse than non-RIC in improving cognitive impairment in people with acute ischaemic stroke and cerebral small vessel disease. There is very low quality evidence that RIC performed no better or no worse than non-RIC in reducing stroke severity (assessed by NIHSS score and infarct volume) in people with acute ischaemic stroke whether or not treated with intravenous thrombolysis. However, further research is likely to have an important impact on our confidence in the above estimations.

\section{Potential biases in the review process}

We carried out a comprehensive search across major databases, and we believe that we identified all relevant studies. In addition, we contacted the study authors for additional data when needed.
However, as less than 10 trials were included this review and all included studies had a small sample size, potential known and unknown biases cannot be excluded. In addition, this metaanalysis was slightly hampered by missing data because of studies reporting outcome data as medians and interquartile ranges. We were unable to obtain raw data for these studies, and this may cause biases to our analyses.

\section{Agreements and disagreements with other studies or reviews}

The findings of our review regarding RIC for preventing ischaemic stroke are different from other meta-analyses in the literature. Two Cochrane Reviews (one of which included 29 studies involving 5392 participants who underwent cardiovascular surgery, and the other four studies involving 232 participants who underwent vascular and endovascular surgery) and a non-Cochrane review (which included 23 studies involving a total of 2200 participants who underwent cardiovascular surgery) analysed the effectiveness of RIC on preventing stroke (Benstoem 2017; Desai 2011; Healy 2014). These three previous reviews concluded that RIC had no effect on stroke. This discrepancy may be attributable to the different study populations included in different reviews. In this review, we analysed the effects of RIC in preventing ischaemic stroke in people with symptomatic intracerebral artery stenosis or undergoing carotid stenting, and all these participants had a high possibility of recurrent stroke or new brain infarct. However, in people undergoing cardiovascular surgery, vascular surgery and endovascular surgery, the incidence of stroke is relatively very low, and many studies found no participants developing this complication.

There has been no previous systematic review of RIC versus non$\mathrm{RIC}$ in treating ischaemic stroke. The evidence in this review that RIC should not be used routinely in treating ischaemic stroke disagrees with the conclusions of the authors of the four included studies. However, due to the flaws identified in the included studies, further studies on the efficacy of RIC for treating ischaemic stroke are welcomed.

\section{AUTHORS' CONCLUSIONS}

\section{Implications for practice}

We found low-quality evidence that remote ischaemic conditioning (RIC) may reduce the risk of recurrent stroke in participants with intracerebral artery stenosis, reduce infarct volume in participants undergoing carotid stenting, and increase dependence or death in participants with acute ischaemic stroke who are undergoing intravenous thrombolysis. However, based on the current lowquality evidence from several small trials, it is difficult to determine whether people with symptomatic intracerebral artery stenosis, acute ischaemic stroke, cerebral small vessel disease, or those undergoing carotid artery stenting will benefit from RIC. Routine use of RIC to prevent or treat ischaemic stroke cannot be supported by the current evidence. Although adverse events were more common with RIC caused by cuff inflation, serious adverse events were not reported.

\section{Implications for research}

Further large-scale trials may be worthwhile to determine the efficacy of RIC in treating acute ischaemic stroke, especially those treated with reperfusion therapy (e.g. intravenous thrombolysis 
and endovascular therapy). Researchers are also encouraged to investigate RIC in other subtypes of cerebrovascular diseases (e.g. haemorrhagic stroke). Furthermore, the optimal dose and the treatment protocol for RIC treatment needs further investigation. The safety of RIC needs to be further determined in adequatelydesigned studies, and further studies should be conducted and reported according to the CONSORT Statement (Schulz 2010).

\section{A C K N OWLEDGEMENTS}

We thank Hazel Fraser (Managing Editor, Cochrane Stroke Group) and Joshua Cheyne (Information Specialist, Cochrane Stroke
Group) for their valuable help during the preparation and revision of this review. We thank Peter Langhorne, Paul Nederkoorn, Aryelly Rodriguez (the Cochrane Stroke Group editors), Jessica Sharp (the Copyeditor), and Dee Shneiderman (consumer reviewer for this review) for their expert advice, helpful comments and suggestions, and assistance with this review. We thank Jin Xu (Deparimen of Library, Xuanwu Hospital, Captial Medical University) for her valuable assistance when preparing and revising the review. 


\section{RE F E R E N C E S}

\section{References to studies included in this review}

England 2016 \{published data only\}

England T, Hedstrom A, O'Sullivan S, Donnelly R, Sprigg N, Bath P. Remote ischaemic conditioning after stroke trial (RECAST). International Journal of Stroke 2016;11 Suppl 4:6.

\section{Hougaard 2014 \{published data only\}}

Hougaard KD, Hjort N, Zeidler D, Sorensen L, Norgaard A, Hansen TM, et al. Remote ischemic perconditioning as an adjunct therapy to thrombolysis in patients with acute ischemic stroke: a randomized trial. Stroke 2014;45(159):67. [PUBMED: PMID: 24203849]

\section{Meng 2012 \{published data only\}}

Meng R, Asmaro K, Meng L, Liu Y, Ma C, Xi C, et al. Upper limb ischemic preconditioning prevents recurrent stroke in intracranial arterial stenosis. Neurology 2012;79(18):1853-61. [PUBMED: PMID: 23035060]

\section{Meng 2015 \{published data only\}}

Meng R, Ding Y, Asmaro K, Brogan D, Meng L, Sui M, et al. Ischemic conditioning is safe and effective for octoand nonagenarians in stroke prevention and treatment. Neurotherapeutics 2015;12(3):667-77. [PUBMED: PMID: 25956401]

\section{Mi 2016 \{published data only\}}

Mi T, Yu F, Ji X, Sun Y, Qu D. The interventional effect of remote ischemic preconditioning on cerebral small vessel disease: a pilot randomized clinical trial. European Neurology 2016;76(1-2):28-34. [PUBMED: PMID: 27351719]

\section{Wang 2017 \{published data only\}}

Wang Y, Meng R, Song H, Liu G, Hua Y, Cui D, et al. Remote ischemic conditioning may improve outcomes of patients with cerebral small-vessel disease. Stroke 2017;48(11):3064-72. [PUBMED: PMID: 29042490 ]

\section{Zhao 2017 \{published data only\}}

Zhao W, Meng R, Ma C, Hou B, Jiao L, Zhu F, et al. Safety and efficacy of remote ischemic preconditioning in patients with severe carotid artery stenosis before carotid artery stenting: a proof-of-concept, randomized controlled trial. Circulation 2017;135(14):1325-35. [PUBMED: PMID: 28174194]

\section{References to studies excluded from this review}

\section{Cho 2017 \{published data only\}}

Cho YJ, Lee EH, Lee K, Kim TK, Hong DM, Chin JH, et al. Longterm clinical outcomes of remote ischemic preconditioning and postconditioning outcome (rispo) trial in patients undergoing cardiac surgery. International Journal of Cardiology 2017;231:84-9.

\section{Coverdale 2017 \{published data only\}}

Coverdale NS, Hamilton A, Petsikas D, McClure RS, Malik P, Milne $\mathrm{B}$, et al. Remote ischemic preconditioning in high-risk cardiovascular surgery patients: a randomized-controlled trial. http://www.semthorcardiovascsurg.com/article/ S1043-0679(17)30238-1/fulltext 2017.

\section{Davies 2013 \{published and unpublished data\}}

Davies WR, Brown AJ, Watson W, McCormick LM, West NEJ, Dutka DP, et al. Remote ischemic preconditioning improves outcome at 6 years after elective percutaneous coronary intervention: the CRISP stent trial long-term follow-up. Circulation: Cardiovascular Interventions 2013;6(3):246-51.

Healy 2015 \{published data only (unpublished sought but not used)\}

Healy DA, Boyle E, McCartan D, Bourke M, Medani M, Ferguson J, et al. A multicenter pilot randomized controlled trial of remote ischemic preconditioning in major vascular surgery. Vascular and Endovascular Surgery 2015;49(8):220-7.

Hoole 2009 \{published data only\}

Hoole SP, Heck PM, Sharples L, Khan SN, Duehmke R, Densem CG, et al. Cardiac remote ischemic preconditioning in coronary stenting (CRISP Stent) study: a prospective, randomized control trial. Circulation 2009;119(6):820-7.

Hudetz 2014 \{published data only\}

Hudetz J, Patterson K, Iqbal Z, Gandhi S, Pagel P. Remote ischemic preconditioning prevents deterioration of short-term postoperative cognitive function after cardiac surgery using cardiopulmonary bypass: results of a pilot investigation. Journal of Cardiothoracic and Vascular Anesthesia 2014;29(2):382-8.

\section{Hyngstrom 2016 \{published and unpublished data\}}

Hyngstrom A, Schmit BD, Gutterman DD, Durand MJ. Ischemic preconditioning as a method to improve motor function post stroke. Archives of Physical Medicine and Rehabilitation 2016;97(10):819 (Abst. 804).

Jing 2011 \{published and unpublished data\} Jing G, Zheng L. Protective effects of remote ischemic preconditioning on cerebral injury in patients undergoing cardiac valve replacement with CPB. Journal of Xian Jiaotong University 2011;32(4):473-6.

\section{Joseph 2015 \{published data only\}}

Joseph B, Pandit V, Zangbar B, Kulvatunyou N, Khalil M, Tang A et al. Secondary brain injury in trauma patients: the effects of remote ischemic conditioning. Journal of Trauma and Acute Care Surgery 2015;78(4):698-705.

\section{Kahlert 2017 \{published data only\}}

Kahlert P, Hildebrandt HA, Patsalis PC, Al-Rashid F, Janosi RA, Nensa F, et al. No protection of heart, kidneys and brain by remote ischemic preconditioning before transfemoral transcatheter aortic valve implantation: interim-analysis of a randomized single-blinded, placebo-controlled, single-center trial. International Journal of Cardiology 2017;231(3):248-54. 
Meng 2017 \{published data only\}

Meng R, Zhao W, Li S, Sui M, Zhou D, Wu W, et al. Long-term regular remote ischemic conditioning promotes arterial collateral opening and reconstruction in patients with intracranial atherosclerotic stenosis. Stroke 2017;48:A76-A76.

\section{NCT01672515 \{unpublished data only\}}

NCT01672515. Protective effects of remote limb ischemic preconditioning on acute cerebral infarction. https:// clinicaltrials.gov/ct2/show/NCT01672515 (accessed 10 January 2018).

\section{Sales 2017 \{published data only\}}

Sales AHA, Barz M, Bette S, Wiestler B, Ryang YM, Meyer B, et al. Impact of ischemic preconditioning on surgical treatment of brain tumors: a single-center, randomized, double-blind, controlled trial. BMC Medicine 2017;15(1):137.

\section{Sloth 2014 \{published data only\}}

Sloth AD, Schmidt MR, Munk K, Kharbanda RK, Redington AN, Schmidt M, et al. Improved long-term clinical outcomes in patients with ST-elevation myocardial infarction undergoing remote ischaemic conditioning as an adjunct to primary percutaneous coronary intervention. European Heart Journal 2014;35(3):168-75

\section{Walsh 2010 \{published data only\}}

Walsh SR, Nouraei SA, Tang TY, Sadat U, Carpenter RH, Gaunt ME. Remote ischemic preconditioning for cerebral and cardiac protection during carotid endarterectomy: results from a pilot randomized clinical trial. Vascular and Endovascular Surgery 2010;44(6):434-9.

\section{Zhong 2013 \{published data only\}}

Zhong H, Gao Z, Chen M, Zhao J, Wang F, Li L, et al. Cardioprotective effect of remote ischemic postconditioning on children undergoing cardiac surgery: a randomized controlled trial. Paediatric Anaesthesia 2013;23(8):726-33.

\section{References to ongoing studies}

\section{NCT02169739 \{unpublished data only\}}

NCT02169739. Remote preconditioning over time to empower cerebral tissue (REM-PROTECT) [Pilot, Randomized, Controlled, Staggered Start, Feasibility Trial of Ischemic Preconditioning, a Promising Novel Treatment for Stroke Prevention]. https:// clinicaltrials.gov/show/NCT02169739 (first received 23 June 2014).

\section{NCT02189928 \{unpublished data only\}}

NCT02189928. REmote iSchemic Conditioning in acUtE BRAin INfarction Study (RESCUE-BRAIN) [Lower Limb Ischaemic Perconditioning in Acute Cerebral Infarction ( $<\mathrm{H} 6)$ : Multicenter Randomized Study With Stratification on IV Thrombolysis and PROBE Design (Prospective Randomized Open Trial With Blinded End-Point)]. https://clinicaltrials.gov/show/ NCT02189928 (first received 15 July 2014).
NCT02323425 \{unpublished data only\}

NCT02323425. Effects of limb ischemic postconditioning in young sICAS (EPIC-sICAS) [Effects of Upper Limb Ischemic Postconditioning on Collateral Circulation in Young Symptomatic Intracranial Atherosclerosis]. https:// clinicaltrials.gov/show/NCT02323425 (first received 23 December 2014).

\section{NCT02534545 \{unpublished data only\}}

NCT02534545. Remote ischemic conditioning for avoiding recurrence of symptomatic intracranial atherosclerotic stenosis (sICAS) [Remote Ischemic Conditioning for Avoiding Recurrence of Ischemic Stroke in Patients With Symptomatic Intracranial Atherosclerotic Stenosis]. https://clinicaltrials.gov/show/ NCT02534545 (first received 27 August 2015).

NCT02779712 \{unpublished data only\}

NCT02779712. Remote ischaemic conditioning after stroke trial (ReCAST-2) [Remote Ischaemic Conditioning After Stroke Trial (ReCAST-2): A Pilot Randomised Controlled Phase II Trial Evaluating Remote Ischaemic Conditioning (RIC) After Hyperacute Stroke 2]. https://clinicaltrials.gov/show/ NCT02779712 (first received 20 May 2016).

NCT03105141 \{unpublished data only\}

NCT03105141. Optimized remote ischemic conditioning (RIC) treatment for patients with chronic cerebral ischemia [The Impact of Repeated Bilateral Limb Remote Ischemic Conditioning on Patients With Chronic Cerebral Ischemia: Establishment of Optimized Algorithm on the Basis of Feasibility, Safety and Efficacy]. https://clinicaltrials.gov/show/ NCT03105141 (first received 7 April 2017).

NCT03208166 \{unpublished data only\}

NCT03208166. Pilot study of pre-ischemic conditioning for intracranial atherosclerosis (PICASSO) [Pilot Study of PreIschemic Conditioning for Intracranial Atherosclerosis]. https:// clinicaltrials.gov/show/NCT03208166 (first received 5 July 2017).

NCT03231384 \{unpublished data only\}

NCT03231384. rt-PA thrombolytic therapy in combination with remote ischemic conditioning for acute ischemic stroke [Safety and Feasibility of Rt-PA Thrombolytic Therapy in Combination With Remote Ischemic Conditioning for Acute Ischemic Stroke(rtPA-RIC1)]. https://clinicaltrials.gov/show/ NCT03231384 (first received 27 July 2017).

\section{Additional references}

\section{Bai 2015}

Bai J, Lyden PD. Revisiting cerebral postischemic reperfusion injury: new insights in understanding reperfusion failure, hemorrhage, and edema. International Journal of Stroke 2015;10(2):143-52.

\section{Benstoem 2017}

Benstoem C, Stoppe C, Liakopoulos OJ, Ney J, Hasenclever D, Meybohm $P$, et al. Remote ischaemic preconditioning for coronary artery bypass grafting (with or without valve surgery). 
Cochrane Database of Systematic Reviews 2017, Issue 5. [DOI: 10.1002/14651858.CD011719.pub3]

\section{Desai 2011}

Desai M, Gurusamy KS, Ghanbari H, Hamilton G, Seifalian AM. Remote ischaemic preconditioning versus no remote ischaemic preconditioning for vascular and endovascular surgical procedures. Cochrane Database of Systematic Reviews 2011, Issue 12. [DOI: 10.1002/14651858.CD008472.pub2]

\section{Feigin 2015}

Feigin VL, Krishnamurthi RV, Parmar P, Norrving B, Mensah GA, Bennett DA, et al. Update on the global burden of ischemic and hemorrhagic stroke in 1990-2013: the GBD 2013 study. Neuroepidemiology 2015;45(3):161-76.

\section{Guyatt 2008}

Guyatt GH, Oxman AD, Vist G, Kunz R, Falck-Ytter Y, AlonsoCoello $P$, et al. GRADE: an emerging consensus on rating quality of evidence and strength of recommendations. $B M \mathrm{~J}$ 2008;336(7650):924-6.

\section{Hahn 2011}

Hahn CD, Manlhiot C, Schmidt MR, Nielsen TT, Redington AN. Remote ischemic per-conditioning: a novel therapy for acute stroke?. Stroke 2011;42(10):2960-2.

\section{Hausenloy 2016}

Hausenloy DJ, Yellon DM. Ischaemic conditioning and reperfusion injury. Nature Reviews Cardiology 2016;13(4):193-209.

\section{Healy 2014}

Healy DA, Khan WA, Wong CS, Moloney MC, Grace PA, Coffey JC, et al. Remote preconditioning and major clinical complications following adult cardiovascular surgery: systematic review and meta-analysis. International Journal of Cardiology 2014;176(1):20-31.

\section{Higgins 2011}

Higgins JPT, Green S (editors). Cochrane Handbook for Systematic Reviews of Interventions Version 5.1.0 [updated March 2011]. The Cochrane Collaboration, 2011. www.cochranehandbook.org.

\section{Hoda 2012}

Hoda MN, Siddiqui S, Herberg S, Periyasamy-Thandavan S, Bhatia $\mathrm{K}$, Hafez SS, et al. Remote ischemic perconditioning is effective alone and in combination with intravenous tissuetype plasminogen activator in murine model of embolic stroke. Stroke 2012;43(10):2794-9.

\section{Lim 2012}

Lim SY, Hausenloy DJ. Remote ischemic conditioning: from bench to bedside. Frontiers in Physiology 2012;3:27.

\section{Menting 2017}

Menting TP, Wever KE, Ozdemir-van Brunschot DMD, Van der Vliet DJA, Rovers MM, Warle M. Ischaemic preconditioning for the reduction of renal ischaemia reperfusion injury.
Cochrane Database of Systematic Reviews 2017, Issue 3. [DOI: 10.1002/14651858.CD010777.pub2]

\section{Murry 1986}

Murry CE, Jennings RB, Reimer KA. Preconditioning with ischemia: a delay of lethal cell injury in ischemic myocardium. Circulation 1986;74(5):1124-36.

\section{Nogueira 2018}

Nogueira RG, Jadhav AP, Haussen DC, Bonafe A, Budzik RF, Bhuva P, et al. Thrombectomy 6 to 24 Hours after Stroke with a Mismatch between Deficit and Infarct. New England Journal of Medicine 2018;378(1):11-21.

\section{Pan 2016}

Pan J, Li X, Peng Y. Remote ischemic conditioning for acute ischemic stroke: dawn in the darkness. Reviews in the Neurosciences 2016;27(5):501-10.

\section{Powers 2018}

Powers WJ, Rabinstein AA, Ackerson T, Adeoye OM, Bambakidis NC, Becker K, et al. 2018 Guidelines for the Early Management of Patients With Acute Ischemic Stroke: A Guideline for Healthcare Professionals From the American Heart Association/American StrokeAssociation. Stroke 2018;49(3):e46e110.

\section{Przyklenk 1993}

Przyklenk K, Bauer B, Ovize M, Kloner RA, Whittaker P. Regional ischemic 'preconditioning' protects remote virgin myocardium from subsequent sustained coronary occlusion. Circulation 1993;87(3):893-9.

\section{Ren 2015}

Ren C, Wang P, Wang B, Li N, Li W, Zhang C, et al. Limb remote ischemic per-conditioning in combination with postconditioning reduces brain damage and promotes neuroglobin expression in the rat brain after ischemic stroke. Restorative Neurology and Neuroscience 2015;33(3):369-79.

\section{Review Manager 2014 [Computer program]}

The Nordic Cochrane Centre, The Cochrane Collaboration. Review Manager (RevMan). Version 5.3. Copenhagen: The Nordic Cochrane Centre, The Cochrane Collaboration, 2014.

\section{Roth 2015}

Roth GA, Forouzanfar MH, Moran AE, Barber R, Nguyen G, Feigin VL, et al. Demographic and epidemiologic drivers of global cardiovascular mortality. New England Journal of Medicine 2015;372(14):1333-41.

\section{Schulz 2010}

Schulz KF, Altman DG, Moher D. CONSORT 2010 statement: updated guidelines for reporting parallel group randomised trials. BMJ 2010;340:c332.

\section{Steiger 2016}

Steiger N, Cifu AS. Primary prevention of stroke. Journal of the American Medical Association 2016;316(6):658-9. 


\section{Wan 2014}

Wan X, Wang W, Liu J, Tong T. Estimating the sample mean and standard deviation from the sample size, median, range and/or interquartile range. BMC Medical Research Methodology 2014;14:135.

\section{Weber 2010}

Weber C. Far from the heart: receptor cross-talk in remote conditioning. Nature Medicine 2010;16(7):760-2.

\section{Writing Group 2016}

Writing Group Members, Mozaffarian D, Benjamin EJ, Go AS, Arnett DK, Blaha MJ, et al. Heart disease and stroke

\section{CHARACTERISTICS OF STUDIES}

Characteristics of included studies [ordered by study ID] statistics-2016 update: a report from the American Heart Association. Circulation 2016;133(4):e38-236.

\section{References to other published versions of this review Zhao 2017}

Zhao W, Zhang J, Sadowsky MG, Meng R, Ding Y, Ji X. Remote ischaemic conditioning for preventing and treating ischaemic stroke. Cochrane Database of Systematic Reviews 2017, Issue 1. [DOI: 10.1002/14651858.CD012503]

\section{England 2016}

Methods Study design: RCT

Generation of the allocation sequence: minimised on age, sex, NIHSS score, and systolic blood pressure

Allocation concealment: web-based, 1:1 allocation

Study grouping: parallel group

Masking: triple-blinded (participants, investigator, outcome assessor)

Analysis method: ITT analysis

Total duration of study: 27 months, from March 2013 to July 2015

\section{Participants}

26 participants (intervention $=13$; placebo control $=13$ )

UK

Single centre: Derby Teaching Hospitals NHS Foundation Trust

Setting: inpatient

Sex: both (65.4\% men, $34.6 \%$ women)

Mean age: 76.2 years old

Inclusion criteria: adults with an ischaemic stroke in the past 24 hours causing arm or leg weakness

Exclusion criteria: $\mathrm{mRS}$ score $>3$, thrombolysis for index event, and significant comorbidity

Participants were randomly assigned to either of 2 groups:

Group 1: RIC group ( $n=13)$, standard medical management plus RIC. RIC consisted of 4 cycles of intermittent limb ischaemia; alternating 5 minutes' inflation $(20 \mathrm{mmHg}$ above systolic blood pressure) and 5 minutes' deflation performed manually using a standard upper arm blood pressure cuff in the nonparetic arm.

Group 2: placebo control group $(n=13)$, standard medical management plus sham RIC. Sham RIC consisted 4 cycles of intermittent limb ischaemia; alternating 5 minutes' inflation $(30 \mathrm{mmHg})$ and 5 minutes' deflation performed manually using a standard upper arm blood pressure cuff in the nonparetic arm.

Concomitant medications: single antiplatelets, blood pressure and cholesterol reduction treatment 
England 2016 (Continued)

Outcomes

Primary outcome: tolerability and feasibility of RIC after acute ischaemic stroke

Secondary outcomes: surrogate markers of efficacy (plasma S100- $\beta$, matrix metalloproteinase-9, troponin T), inflammation (C-reactive protein), and other putative biomarker (heat shock proteins and endocannabinoids). Transcranial Doppler was performed as a continuous beat-to-beat recording during the intervention; central pressures measured with Sphygmocor. Serious adverse events, mRS score, impairment (NIHSS score and motricity index), BI, extended activities of daily living, Zung Depression Scale, and cognition (MMSE) were measured at day 90.

Notes
Follow-up: $100 \%$
Demographic: adequately reported and similar
Principle investigator: Timothy J England, PhD, FRCP
Funding: British Medical Association Vera Down Grant
Notable authors' disclosures: no

\section{Risk of bias}

\begin{tabular}{|c|c|c|}
\hline Bias & Authors' judgement & Support for judgement \\
\hline $\begin{array}{l}\text { Random sequence genera- } \\
\text { tion (selection bias) }\end{array}$ & Low risk & The study minimised on age, sex, NIHSS score, and systolic blood pressure. \\
\hline $\begin{array}{l}\text { Allocation concealment } \\
\text { (selection bias) }\end{array}$ & Low risk & Web-based, and 1:1 allocation \\
\hline $\begin{array}{l}\text { Blinding of participants } \\
\text { and personnel (perfor- } \\
\text { mance bias) } \\
\text { All outcomes }\end{array}$ & Low risk & $\begin{array}{l}\text { The intervention group received RIC procedure and the control group received } \\
\text { sham procedure. }\end{array}$ \\
\hline $\begin{array}{l}\text { Blinding of outcome as- } \\
\text { sessment (detection bias) } \\
\text { All outcomes }\end{array}$ & Low risk & $\begin{array}{l}\text { This was a single-centre, randomised, outcome-blinded, placebo-controlled } \\
\text { trial. }\end{array}$ \\
\hline $\begin{array}{l}\text { Incomplete outcome data } \\
\text { (attrition bias) } \\
\text { All outcomes }\end{array}$ & Low risk & No missing outcome data \\
\hline $\begin{array}{l}\text { Selective reporting (re- } \\
\text { porting bias) }\end{array}$ & Low risk & $\begin{array}{l}\text { The study protocol was available and all of the study's prespecified outcomes } \\
\text { had been reported in the pre-specified way. }\end{array}$ \\
\hline Other bias & Low risk & No other bias identified \\
\hline
\end{tabular}

\section{Hougaard 2014}

Study design: RCT
Randomisation: drawing from a large number of sealed opaque envelopes
Allocation concealment: sealed opaque envelopes
Study grouping: parallel group


Hougaard 2014 (Continued)

Masking: single-blinded (outcome assessor)

Analysis method: PP analysis

Total duration of study: 19 months, from June 2009 to January 2011

Participants 285 participants (intervention =160; control =125)
Denmark
Single centre: Aarhus University Hospital
Setting: emergency
Sex: both (57\% men, 59\% women)
Mean age: 66.9 years old
Inclusion criteria: eligible patients were $\geq 18$ years of age with symptoms of acute stroke according
to the prehospital services assessment, as per standardised national guidelines (modified Face, Arm,
Speech Test (FAST) criteria).
Exclusion criteria: contraindications to rtPA or MRI, known hypersensitivity to Gadovist or any of its in-
gredients, acute or chronic severe renal impairment, and people with a non-stroke diagnosis

Interventions Participants were randomly assigned to either of 2 groups:

Group 1: RIC group ( $n=81$ ), intravenous thrombolysis plus RIC. RIC was induced by 4 inflations of a standard upper limb blood pressure cuff to either $200 \mathrm{mmHg}$ or $25 \mathrm{mmHg}$ above the patient's systolic blood pressure, each lasting 5 minutes and separated by 5 minutes of cuff deflation

Group 2: control group $(n=68)$, intravenous thrombolysis alone

Concomitant medications: standard care according to national guidelines for the treatment of acute ischaemic stroke by intravenous thrombolysis

Outcomes Primary outcome: the penumbra salvage

Secondary outcomes: final infarct size, infarct growth, and clinical outcome at 3 months

Other outcomes: risk of infarction in treated and untreated tissue according to the severity of the level of hypoperfusion at admission

Notes

External blinded review of outcomes

Follow-up: $52.3 \%$

Demographic: inadequately reported

Principle investigator: Kristina Dupont Hougaard, MD

Funding: the study was funded by grants from the Danish National Research Foundation, the Aase and Ejnar Danielsen Foundation, and the Tryg Foundation. Bayer AG supplied the contrast media for perfusion scans.

Notable disclosures: 2 authors are shareholders in CellAegis Devices Inc, who made the RIC device in the study.

\section{Risk of bias}

\section{Bias}

Authors' judgement Support for judgement 
Hougaard 2014 (Continued)

Random sequence genera- Low risk Randomisation was performed by drawing from a large number of sealed tion (selection bias) opaque envelopes.

Allocation concealment Unclear risk

Sealed opaque envelopes were used, but whether envelopes were sequentially (selection bias) numbered is unclear.

Blinding of participants High risk

and personnel (perfor-

The participants were not blinded to the treatment protocol.

mance bias)

All outcomes

Blinding of outcome as-
sessment (detection bias) $\quad$ Low risk All outcomes assessors were blinded to treatment allocation.

sessment (detection bias)

All outcomes

\begin{tabular}{|c|c|c|}
\hline $\begin{array}{l}\text { Incomplete outcome data } \\
\text { (attrition bias) }\end{array}$ & High risk & $\begin{array}{l}\text { "As-treated" analysis was done with substantial departure of the intervention } \\
\text { received from that assigned at randomisation. }\end{array}$ \\
\hline
\end{tabular}

All outcomes

\begin{tabular}{lll}
\hline $\begin{array}{l}\text { Selective reporting (re- } \\
\text { porting bias) }\end{array}$ & Low risk & $\begin{array}{l}\text { The study protocol was available and all of the study's prespecified outcomes } \\
\text { had been reported in the prespecified way. }\end{array}$ \\
\hline Other bias & High risk & $\begin{array}{l}\text { 2 authors were shareholders in CellAegis Devices Inc where the devices used in } \\
\text { this study were made. }\end{array}$
\end{tabular}

Meng 2012

\begin{tabular}{|c|c|}
\hline \multirow[t]{7}{*}{ Methods } & Study design: RCT \\
\hline & Generation of the allocation sequence: random number \\
\hline & Allocation concealment: unclear \\
\hline & Study grouping: parallel group \\
\hline & Masking: single-blinded (outcome assessor) \\
\hline & Analysis method: PP analysis \\
\hline & Total duration of study: unclear \\
\hline \multirow[t]{7}{*}{ Participants } & 103 participants (intervention $=51 ;$ control $=52$ ) \\
\hline & China \\
\hline & 2 centres \\
\hline & Setting: clinic and inpatient \\
\hline & Sex: both ( $58.8 \%$ men, $41.2 \%$ women) \\
\hline & Mean age: 60.6 years old \\
\hline & $\begin{array}{l}\text { Inclusion criteria: } 1 \text { ) intracranial artery stenosis (measured as } 50 \% \text { by angiography or } 70 \% \text { by ultra- } \\
\text { sound, CTA or MRA) patient with corresponding stroke and TIA within } 30 \text { days from the start of the } \\
\text { study; } 2 \text { ) age range } 18 \text { to } 80 \text { years; } 3 \text { ) NIHSS score } 0-15 \text {, and mRS score } 2-4 \text { (mRS } 0-1 \text { were excluded); } 4 \text { ) } \\
\text { ABCD2 score } 6-7 ; 5 \text { ) stable vital signs, normal hepatic and renal functions; } 6 \text { ) no haemorrhagic tenden- } \\
\text { cy; 7) Trial of Org } 10172 \text { in Acute Stroke Treatment (TOAST) } 1 \text { subtype }\end{array}$ \\
\hline
\end{tabular}


Meng 2012 (Continued)

Interventions

Participants were randomly assigned to one of 2 groups:

Group 1: RIC group ( $n=38)$, standard medical management plus RIC. RIC consisted of 5 cycles of bilateral upper limbs ischaemia (induced by inflating tourniquets to $200 \mathrm{mmHg}$ ) for 5 minutes followed by reperfusion for another 5 minutes, performed twice daily for a total of 300 consecutive days.

Group 2: control group $(n=30)$, standard medical management alone

Concomitant medications: antiplatelet agents, lipid control agents, and antidiabetic agents (if necessary)

\begin{tabular}{|c|c|}
\hline Outcomes & $\begin{array}{l}\text { Primary outcome: time point of the first stroke recurrence event } \\
\text { Secondary outcome: time to which mRS recovers to } 0-1 \\
\text { Other outcomes: RIC treatment intolerance }\end{array}$ \\
\hline Notes & $\begin{array}{l}\text { External blinded review of outcomes } \\
\text { Follow-up: } 66 \% \\
\text { Demographic: adequately reported and similar } \\
\text { Principle investigator: Xunming Ji, MD, PhD } \\
\text { Funding: this study was sponsored by the National Natural Science Foundation ( } 30870854 \text { and } \\
30770741 \text {, China) and the Foundation of the Beijing High Level Health Systems Talented Technical Per- } \\
\text { sonnel Program (2009-03-02, China). } \\
\text { Conflicts of interest of authors: the corresponding author is one of the inventors of the electric au- } \\
\text { to-control device used in the study. }\end{array}$ \\
\hline
\end{tabular}

\section{Risk of bias}

Bias Authors' judgement Support for judgement

Random sequence genera- Low risk All participants were randomly divided into 2 groups according to a random tion (selection bias) number.

Allocation concealment $\quad$ Unclear risk $\quad$ The method of concealment was not described.
(selection bias)

\begin{tabular}{|c|c|c|}
\hline $\begin{array}{l}\text { Blinding of participants } \\
\text { and personnel (perfor- } \\
\text { mance bias) } \\
\text { All outcomes }\end{array}$ & High risk & $\begin{array}{l}\text { Doctors and participants were unmasked to the treatment protocol and allo- } \\
\text { cation. }\end{array}$ \\
\hline $\begin{array}{l}\text { Blinding of outcome as- } \\
\text { sessment (detection bias) } \\
\text { All outcomes }\end{array}$ & Low risk & $\begin{array}{l}\text { Investigators responsible for evaluating the results of NIHSS, mRS scores, TCD, } \\
\text { MRI, and SPECT, and the examiners who were responsible for testing plasma } \\
\text { samples of the participants were masked to the treatment protocol and alloca- } \\
\text { tion. }\end{array}$ \\
\hline
\end{tabular}

\begin{tabular}{lll}
\hline $\begin{array}{l}\text { Incomplete outcome data } \\
\text { (attrition bias) }\end{array}$ & High risk & $35(33.98 \%)$ participants lost to follow-up \\
All outcomes & \\
\hline $\begin{array}{l}\text { Selective reporting (re- } \\
\text { porting bias) }\end{array}$ & Low risk & Study authors published findings on all the predefined study outcomes.
\end{tabular}


Meng 2012 (Continued) $\begin{array}{ll}\text { Other bias } \quad \text { High risk } & \text { The corresponding author is one of the inventors of the electric autocontrol } \\ \text { RIC device used in the study. }\end{array}$

Generation of the allocation sequence: computer-generated blocked randomisation technique

Allocation concealment: numbered, opaque, sealed envelopes

Study grouping: parallel group

Masking: triple-blinded (participants, investigator, outcome assessor)

Analysis method: PP analysis

Total duration of study: from 2008 to 2012

\section{Participants}

79 participants (intervention $=40 ;$ control $=39$ )

China

Single centre: Xuanwu Hospital, Capital Medical University

Setting: clinic and inpatient

Sex: both (62.1\% men, $37.9 \%$ women)

Mean age: 83.8 years old

Inclusion criteria: 1 ) intracranial arterial stenosis patients (at least $70 \%$ assessed by MRA or CTA) presented with ischaemic stroke or TIA within 7 days; 2) age 80-95 years; 3 ) NIHSS score of $0-15$, and mRS score of 2-4; 4) ABCD2 score of 6-7;5) stable vital signs, and normal hepatic and renal function; 6) no haemorrhagic tendency; 7) TOAST (Trial of Org 10172 in Acute Stroke Treatment) subtype 1 of stroke

Group 1: RIC group $(n=30)$, standard medical management plus RIC treatment. RIC treatment consisted of 5 cycles of simultaneous bilateral upper arm ischaemia for 5 minutes followed by reperfusion for another 5 minutes, performed twice daily for 180 consecutive days. The RIC procedure was performed using an electric autocontrol device with cuffs that inflated to a pressure of $200 \mathrm{mmHg}$ during the ischaemic period.

Group 2: control group $(n=28)$, standard medical management sham RIC treatment. Sham RIC treatment consisted of 5 cycles of bilateral upper arm ischaemia for 5 minutes followed by reperfusion for another 5 minutes, performed twice daily for 180 consecutive days. The sham procedure was performed using an electric autocontrol device with cuffs that inflated to a pressure of $30 \mathrm{mmHg}$ during the ischaemic period.

Concomitant medications: either clopidogrel $75 \mathrm{mg} /$ day in combination with atorvastatin $20 \mathrm{mg} /$ day or aspirin $100 \mathrm{mg} /$ day and clopidogrel $75 \mathrm{mg} /$ day in combination with atorvastatin $20 \mathrm{mg} /$ day development of symptomatic or asymptomatic upper extremity deep vein thrombosis as detected by a vascular ultrasound; 3) elevation of plasma myoglobin levels beyond normal limits after RIC treatment; 4) symptomatic haemorrhagic transformation after cerebral infarction; 5) participants not tolerating RIC treatment 
Clinical outcomes: 1) stroke and TIA recurrence during the 180 consecutive days of treatment were defined as primary outcome endpoints; 2 ) improvement of the NIHSS score ( $\geq 8$ points or $\leq 1$ point from the baseline) or a mRS of 0-2 were the secondary outcomes

Blood evaluation outcome: dynamic changes in plasma levels of hsCRP, leukocyte count, fibrinogen, Ddimer, PAI-1 TPA, and platelet aggregation rates in 30 days of RIC and sham RIC

External blinded review of outcomes
Follow up: $73.4 \%$.
Demographic: adequately reported and similar
Principle investigator: Xunming Ji, MD, PhD
Funding: this study was sponsored by the National Natural Science Foundation (81371289), China,
The Foundation of the Beijing High Level Health Systems (Talented Technical Personnel Program
2009-03-02), China; and The National Key Department of Neurology funded by Chinese Health and
Family Planning Committee
Notable disclosures: the corresponding author is one of the inventors of the electric auto-control de-
vice used in the study.

\section{Risk of bias}

\begin{tabular}{lll}
\hline Bias & Authors' judgement & Support for judgement \\
\hline $\begin{array}{l}\text { Random sequence genera- } \\
\text { tion (selection bias) }\end{array}$ & Low risk & $\begin{array}{l}\text { Random sequence was generated by using a computer-generated blocked } \\
\text { randomisation technique. }\end{array}$ \\
\hline $\begin{array}{l}\text { Allocation concealment } \\
\text { (selection bias) }\end{array}$ & Low risk & $\begin{array}{l}\text { The allocation sequence is computer generated and subsequently concealed } \\
\text { in consecutively numbered, opaque, sealed envelopes. }\end{array}$ \\
\hline $\begin{array}{l}\text { Blinding of participants } \\
\begin{array}{l}\text { and personnel (perfor- } \\
\text { mance bias) } \\
\text { All outcomes }\end{array}\end{array}$ & Low risk & Sham RIC was used as placebo control. \\
\hline
\end{tabular}

\begin{tabular}{|c|c|c|}
\hline $\begin{array}{l}\text { Blinding of outcome as- } \\
\text { sessment (detection bias) } \\
\text { All outcomes }\end{array}$ & Low risk & $\begin{array}{l}\text { The research assistants responsible for local skin status evaluations, blood } \\
\text { pressure measurements, heart rate measurements, plasma sample assays, } \\
\text { and neuroradiology interpretation were blinded to the treatment assignment. }\end{array}$ \\
\hline
\end{tabular}

Incomplete outcome data High risk 21/79 (26.6\%) participants lost to follow-up

(attrition bias)

All outcomes

\begin{tabular}{lll}
\hline $\begin{array}{l}\text { Selective reporting (re- } \\
\text { porting bias) }\end{array}$ & Low risk & Authors published findings on all the predefined study outcomes \\
\hline Other bias & High risk & $\begin{array}{l}\text { The corresponding author is one of the inventors of the electric autocontrol } \\
\text { RIC device used in the study. }\end{array}$ \\
\hline
\end{tabular}

Mi 2016

\begin{tabular}{ll}
\hline Methods & Study design: RCT \\
Generation of the allocation sequence: computerised random number sequence \\
Allocation concealment: unclear
\end{tabular}


Mi 2016 (Continued)

\author{
Study grouping: parallel group \\ Masking: double-blinded (investigator, outcome assessor) \\ Analysis method: ITT analysis \\ Total duration of study: 14 months, from to August 2012 to October 2013
}

Participants 17 participants (intervention = 9; placebo control = 8)
China
Single centre: Xuanwu Hospital, Capital Medical University
Setting: clinic and inpatient
Sex: both (41.2\% men, 58.8\% women)
Mean age: 63.5 years old
Inclusion criteria: people diagnosed with small vessel disease
Exclusion criteria: 1 ) history of intracranial haemorrhage; 2) significant bleeding in other parts of the
body; 3) history of atrial fibrillation; 4) history of myocardial infarction within 6 month; 5) Moyamoya
disease, hereditary small vessel disorders (e.g. CADASIL or Fabry) and mitochondrial encephalomyopa-
thy; 6 ) significant bleeding coagulation dysfunction; 7 ) severe liver/kidney disease, cancer, or critical ill-
ness pertaining to internal medicine and surgery

Interventions

Participants were randomly assigned to either of 2 groups:

Group 1: RIC group ( $n=9)$, standard medical management plus RIC. RIC was achieved using an automated cuff inflator placed on bilateral arms that was inflated to $200 \mathrm{mmHg}$. The cuff remained inflated for 5 minutes, followed by 5 minutes of reperfusion. Each preconditioning session consisted of 5 cycles of ischaemia/reperfusion. All participants were required to receive RIC treatment twice daily for 1 year.

Group 2: control group $(n=8)$, sham RIC was achieved using an automated cuff inflator placed on bilateral arms that was inflated to $50 \mathrm{mmHg}$. The cuff remained inflated for 5 minutes, followed by $5 \mathrm{~min}-$ utes of reperfusion. Each conditioning session consisted of 5 cycles of ischaemia/reperfusion. All participants were required to receive RIC treatment twice daily for 1 year.

Concomitant medications: unclear

Outcomes

Primary outcome: changes in brain lesions evaluated by the number of lacunar infarctions and white matter lesions volume

Secondary outcomes: changes in cognitive functions, cerebral haemodynamics, and the Dizziness Handicap Inventory scores

Notes

External blinded review of outcomes

Follow up: $100 \%$

Demographic: inadequately reported

Principle investigator: Xunming Ji, Yongxin Sun, MD, PhD

Funding: Talent Cultivation Foundation, the High-Level Health Technology Talent Construction Program of the Beijing Municipal Health Bureau (008-0019); the Talent Cultivation Foundation, Beijing Outstanding Talents Cultivation Fund, Beijing Municipal Committee (2012D005018000007); and the Natural Science Foundation, Shanghai Jiao Tong University School of Medicine (YZ1006)

Conflicts of interest of authors: the authors report no disclosure. 
Mi 2016 (Continued)

\section{Risk of bias}

\begin{tabular}{|c|c|c|}
\hline Bias & Authors' judgement & Support for judgement \\
\hline $\begin{array}{l}\text { Random sequence genera- } \\
\text { tion (selection bias) }\end{array}$ & Low risk & $\begin{array}{l}\text { Randomisation was generated using a computerised random number se- } \\
\text { quence. }\end{array}$ \\
\hline $\begin{array}{l}\text { Allocation concealment } \\
\text { (selection bias) }\end{array}$ & Unclear risk & $\begin{array}{l}\text { "Participant assignments were sealed and handed to specialized staff", but it } \\
\text { remains unclear whether the assignments were sequentially numbered. }\end{array}$ \\
\hline $\begin{array}{l}\text { Blinding of participants } \\
\text { and personnel (perfor- } \\
\text { mance bias) } \\
\text { All outcomes }\end{array}$ & Low risk & Sham RIC was used as placebo control. \\
\hline $\begin{array}{l}\text { Blinding of outcome as- } \\
\text { sessment (detection bias) } \\
\text { All outcomes }\end{array}$ & Low risk & $\begin{array}{l}\text { The outcomes assessors were blinded to the treatment protocol and assign- } \\
\text { ment. }\end{array}$ \\
\hline $\begin{array}{l}\text { Incomplete outcome data } \\
\text { (attrition bias) } \\
\text { All outcomes }\end{array}$ & Low risk & No participant was lost to follow-up. \\
\hline $\begin{array}{l}\text { Selective reporting (re- } \\
\text { porting bias) }\end{array}$ & Low risk & Authors published findings on all the predefined study outcomes. \\
\hline Other bias & High risk & $\begin{array}{l}\text { One author is one of the inventors of the electric autocontrol RIC device used } \\
\text { in the study. }\end{array}$ \\
\hline
\end{tabular}

Wang 2017

Methods

Study design: RCT

Generation of the allocation sequence: unclear

Allocation concealment: unclear

Study grouping: parallel group

Masking: double-blinded (investigator, outcome assessor)

Analysis method: PP analysis

Total duration of study: 35 months, from to July 2012 to June 2015

Participants

36 participants (intervention $=18$; placebo control $=18$ )

China

Single centre: Xuanwu Hospital, Capital Medical University

Setting: clinic and inpatient

Sex: both (50\% men, 50\% women)

Mean age: 63.1 years old

Inclusion criteria: 1) Han Chinese, aged from 45 to 80 years; 2) patient or caregiver report of cognitive decline with regard to memory or other cognitive domains lasting for at least 3 months; 3 ) neither nor- 
Wang 2017 (Continued)

mal nor demented on the basis of the criteria of the Diagnostic and Statistical Manual of Mental Disorders (Fourth Edition) with normal or slightly impaired activities of daily living, with MMSE score of $\geq 20$ (primary school) or $\geq 24$ (junior school or above) and MoCA score of $\leq 26 ; 4$ ) the presence of lacunes or WMHs on MRI according to Standards for Reporting Vascular Changes on Neuroimaging criteria; 5) the absence of vascular narrowing $>50 \%$ luminal diameter that could cause haemodynamic changes (mean flow velocity $>90 \mathrm{~cm} / \mathrm{s}$ for the intracranial internal carotid artery, $100 \mathrm{~cm} / \mathrm{s}$ for the middle carotid artery, $>80 \mathrm{~cm} / \mathrm{s}$ for basilar artery or vertebral artery) measured by TCD according to the Stroke Outcomes and Neuroimaging of Intracranial Atherosclerosis criteria

Exclusion criteria: 1) history of intracranial haemorrhage or significant bleeding in other parts of the body; 2) history of atrial fibrillation or myocardial infarction within 6 months; 3) hereditary or inflammatory small-vessel disorders; 4) significant bleeding disorder; 5) history of brain tumours, psychoses, or acute stroke within 6 months of symptom onset; 6 ) severe renal or hepatic disease, cancer, or critical illness; 7) systolic blood pressure $>200 \mathrm{mmHg}$ with medication; 8) any soft tissue or vascular injury and any disease of the extremities that would contraindicate RIC; 9) no informed consent; 10) failure to complete the entire treatment regimen (the participant's completion rate was $>80 \%$ of the RIC or sham-RIC treatments for 1 year or the participant was excluded from the final analysis)

Interventions $\quad$ Participants were randomly assigned to either of 2 groups:

Group 1: RIC group ( $n=18)$, standard medical management plus RIC. RIC was achieved using an automated cuff inflator placed on bilateral arms that was inflated to $200 \mathrm{mmHg}$. The cuff remained inflated for 5 minutes, followed by 5 minutes of reperfusion. Each preconditioning session consisted of 5 cycles of ischaemia/reperfusion. All participants were required to receive RIC treatment twice daily for 1 year.

Group 2: control group $(n=18)$, sham RIC was achieved using an automated cuff inflator placed on bilateral arms that was inflated to $50 \mathrm{mmHg}$. The cuff remained inflated for 5 minutes, followed by $5 \mathrm{~min}$ utes of reperfusion. Each conditioning session consisted of 5 cycles of ischaemia/reperfusion. All participants were required to receive RIC treatment twice daily for 1 year.

Concomitant medications: antiplatelet agents, statins, and vascular risk factors modification

Outcomes Primary outcome: the change of brain lesions measured on MRI fluid-attenuated inversion recovery

Secondary outcomes: changes of cognitive function measured by MMSE and MoCA, plasma biomarkers, cerebral haemodynamic parameters measured by TCD both at baseline and at the end of 1-year

Notes

External blinded review of outcomes

Follow up: $83.3 \%$

Demographic: adequately reported

Principle investigator: Xunming Ji, MD, PhD

Funding: National Science Foundation for Distinguished Young Scholars (81325007), Beijing Municipal Administration of Hospitals' Mission Plan (20150802), National Natural Science Foundation of China (81371289) and Scientific Research Project of Beijing Municipal Education Commission (KM20160025015)

Conflicts of interest of authors: the authors report no disclosure.

\section{Risk of bias}

\begin{tabular}{lll}
\hline Bias & Authors' judgement & Support for judgement \\
\hline $\begin{array}{l}\text { Random sequence genera- } \\
\text { tion (selection bias) }\end{array}$ & Unclear risk & $\begin{array}{l}\text { Randomisation was performed by an independent statistician, but the details } \\
\text { were not reported. }\end{array}$ \\
\hline $\begin{array}{l}\text { Allocation concealment } \\
\text { (selection bias) }\end{array}$ & Unclear risk & $\begin{array}{l}\text { "Participant assignments were sealed and handed to specialized staff", but it } \\
\text { remains unclear whether the assignments were sequentially numbered }\end{array}$
\end{tabular}


Wang 2017 (Continued)

Blinding of participants Low risk Sham intervention was used and participants were blinded to the treatment and personnel (perforprotocol. mance bias)

All outcomes

\begin{tabular}{|c|c|c|}
\hline $\begin{array}{l}\text { Blinding of outcome as- } \\
\text { sessment (detection bias) }\end{array}$ & Low risk & $\begin{array}{l}\text { The outcomes assessors were blinded to the treatment protocol and assign- } \\
\text { ment. }\end{array}$ \\
\hline
\end{tabular}

All outcomes

Incomplete outcome data High risk

Approximately $17 \%$ participants lost to follow-up

(attrition bias)

All outcomes

\begin{tabular}{lll}
\hline $\begin{array}{l}\text { Selective reporting (re- } \\
\text { porting bias) }\end{array}$ & Low risk & Authors published findings on all the predefined study outcomes. \\
\hline Other bias & High risk & $\begin{array}{l}\text { One author is one of the inventors of the electric autocontrol RIC device used } \\
\text { in the study. }\end{array}$
\end{tabular}

Zhao 2017

Methods

Study design: RCT

Generation of the allocation sequence: computer-generated list in randomly sequenced blocks of 6

Allocation concealment: numbered, sealed, opaque envelopes

Study grouping: parallel group

Masking: single-blinded (outcome assessor)

Analysis method: ITT and PP analysis

Total duration of study: 29 months, from to 1 August 2012 to 20 December 2014

Participants 189 participants (intervention =63; control = 63; sham intervention =63)
China
Single centre: Xuanwu Hospital, Capital Medical University
Setting: clinic and inpatient
Sex: both (71.4\% men, $28.6 \%$ women)
Mean age: 66.6 years old
Inclusion criteria: 1$) \geq 18$ years of age; (2) tolerance to any of the necessary medications, including
clopidogrel, aspirin, and statins; 3) ability to complete a brain MRI examination; 4) a negative pregnan-
cy test within 7 days before randomisation for any woman with childbearing potential; 5) stable vital
signs, and normal renal and hepatic functions; 6 ) participant or his or her legally authorised represen-
tative was able to provide an informed consent

Interventions

Participants were randomly assigned to one of 3 groups:

Group 1: RIC group ( $n=63$ ), standard medical management plus RIC. RIC was performed twice daily for 2 weeks before carotid stenting. The RIC consisted of 5 cycles of simultaneous bilateral upper arm ischaemia for 5 minutes followed by reperfusion for another 5 minutes. The procedure was performed using an electric autocontrol device with cuffs that inflated to a pressure of $200 \mathrm{mmHg}$ during the ischaemic period 
Zhao 2017 (Continued)

Group 2: control group $(n=63)$, standard medical management alone

Group 3: sham RIC group ( $n=63)$, standard medical management plus sham RIC. Sham RIC procedure during which bilateral upper arm cuffs were inflated to a pressure of $60 \mathrm{mmHg}$ for 5 minutes, followed by 5 minutes of relaxation of the cuffs, for a total of 5 cycles twice daily until the day before carotid stenting

Concomitant medications: antiplatelets and statins were mandatory. Administration of antihypertensive agents and antidiabetic agents were elective at discretion of the treating physician.

Outcomes

Primary outcome: the presence of $\geq 1$ new brain lesions on DWI within 48 hours after CAS and the incidence of clinical events (i.e. ischaemic stroke, TIA, acute myocardial infarction, haemorrhagic stroke, hyperperfusion syndrome, and death) within 6 months after CAS

Secondary outcomes: the number and volume of new brain lesions on DWI within 48 hours; the changes in plasma hs-CRP levels, NSE levels, and S-100B levels; and adverse events within 6 months

Other outcomes: 1) elevation of plasma NSE and S-100B levels beyond normal limits after RIC and sham RIC procedure; 2 ) inability to tolerate RIC or sham RIC procedure that leads to discontinuation from the study; 3 ) objective signs of tissue or neurovascular injury resulting from RIC and sham RIPC procedure

Notes
Follow up: $85.7 \%$ for the primary outcome
Demographic: adequately reported and similar
Principle investigator: Xunming Ji, MD, PhD
Funding: this study was funded by The National Science Fund for Distinguished Young Scholars (No.
81325007), Chang Jiang Scholars Program (No. T2014251), and Beijing Municipal Science and Technol-
ogy Commission (No. Z141107001514006).
Conflicts of interest of authors: the corresponding author is one of the inventors of the electric auto-
control device used in the study.

\section{Risk of bias}

\begin{tabular}{lll}
\hline Bias & Authors' judgement & Support for judgement \\
\hline $\begin{array}{l}\text { Random sequence genera- } \\
\text { tion (selection bias) }\end{array}$ & Low risk & $\begin{array}{l}\text { Participants were randomised by a computer-generated list in randomly se- } \\
\text { quenced blocks of } 6 .\end{array}$ \\
\hline $\begin{array}{l}\text { Allocation concealment } \\
\text { (selection bias) }\end{array}$ & Low risk & Participants were enrolled according to numbered, sealed, opaque envelopes. \\
\hline $\begin{array}{l}\text { Blinding of participants } \\
\text { and personnel (perfor- } \\
\text { mance bias) }\end{array}$ & High risk & $\begin{array}{l}\text { The participants and the treating physicians were not blinded to the treat- } \\
\text { ment. }\end{array}$ \\
\hline $\begin{array}{l}\text { Blinding of outcome as- } \\
\text { sessment (detection bias) } \\
\text { All outcomes }\end{array}$ & Low risk & Outcomes assessors were blinded to the treatment protocol. \\
\hline $\begin{array}{l}\text { Incomplete outcome data } \\
\text { (attrition bias) } \\
\begin{array}{l}\text { All outcomes } \\
\hline\end{array}\end{array}$ & High risk & 27 (14.3\%) participants were lost for the analysis of primary outcomes. \\
\hline
\end{tabular}


Zhao 2017 (Continued)

\begin{tabular}{lll}
$\begin{array}{l}\text { Selective reporting (re- } \\
\text { porting bias) }\end{array}$ & Low risk & All primary and secondary outcomes that are prespecified have been reported. \\
\hline Other bias & High risk & $\begin{array}{l}\text { The corresponding author is one of the inventors of the electric autocontrol } \\
\text { RIC device used in the study. }\end{array}$
\end{tabular}

CAS: carotid artery stenting

CTA: computed tomography angiography

DWI: diffusion weighted imaging

ITT: intention-to-treat

MoCA: Montreal Cognitive Assessment

MMSE: Mini Mental State Examination

MRA: magnetic resonance angiography

MRI: magnetic resonance imaging

mRS: modified Rankin Scale

NIHSS: National Institutes of Health Stroke Scale

PP: per protocol

$\mathrm{RCT}$ : randomised controlled trial

RIC: remote ischaemic conditioning

rtPA: recombinant tissue plasminogen activator

SPECT: single-photon emission computed tomography

TCD: transcranial Doppler

TIA: transient ischaemic attack

WMHs: white matter hyperintensities

Characteristics of excluded studies [ordered by study ID]

\begin{tabular}{|c|c|}
\hline Study & Reason for exclusion \\
\hline Cho 2017 & Study randomised people undergoing cardiac surgery \\
\hline Coverdale 2017 & Study randomised people undergoing cardiovascular surgery \\
\hline Davies 2013 & $\begin{array}{l}\text { Study randomised acute coronary syndrome patients treated with percutaneous coronary inter- } \\
\text { vention }\end{array}$ \\
\hline Healy 2015 & $\begin{array}{l}\text { Study randomised people treated with major vascular surgery, including carotid endarterectomy. } \\
\text { We contacted the study authors to ask if they could provide data on patients treated with carotid } \\
\text { endarterectomy but we did not receive a response. }\end{array}$ \\
\hline Hoole 2009 & $\begin{array}{l}\text { Study randomised acute coronary syndrome patients treated with percutaneous coronary inter- } \\
\text { vention }\end{array}$ \\
\hline Hudetz 2014 & RIC was used in people undergoing cardiac surgery \\
\hline Hyngstrom 2016 & $\begin{array}{l}\text { RIC was used in both healthy volunteers and people with ischaemic cerebrovascular disease but } \\
\text { did not compare RIC with sham RIC or control. }\end{array}$ \\
\hline Jing 2011 & Laboratory tissue study. After emailing the authors, there was no determination of stroke. \\
\hline Joseph 2015 & Not an RCT \\
\hline Kahlert 2017 & Study randomised people treated with transfemoral transcatheter aortic valve implantation \\
\hline Meng 2017 & $\begin{array}{l}\text { This RCT investigated the effects of RIC on cerebral collaterals and the underlying mechanisms in } \\
\text { people with symptomatic intracranial atherosclerotic stenosis, instead of ischaemic stroke. }\end{array}$ \\
\hline
\end{tabular}




\begin{tabular}{ll}
\hline Study & Reason for exclusion \\
\hline NCT01672515 & $\begin{array}{l}\text { The recruitment status of this study is unknown. The completion date has passed and the status } \\
\text { has not been verified in more than } 2 \text { years. No paper or results have been published. }\end{array}$ \\
\hline Sales 2017 & $\begin{array}{l}\text { This study evaluated the effect of RIC for preventing new postoperative ischaemic lesions, but is- } \\
\text { chaemic stroke was not included in the outcome assessments. }\end{array}$ \\
\hline Sloth 2014 & $\begin{array}{l}\text { Study randomised acute coronary syndrome patients treated with percutaneous coronary inter- } \\
\text { vention }\end{array}$ \\
\hline Walsh 2010 & Ischaemic stroke was not included in the outcome assessment \\
\hline Zhong 2013 & Study randomised people treated with cardiac surgery \\
\hline
\end{tabular}

RCT: randomised controlled trial

RIC: remote ischaemic conditioning

Characteristics of ongoing studies [ordered by study ID]

NCT02169739

Trial name or title

Remote preconditioning over time to empower cerebral tissue (REM-PROTECT)

Methods

\author{
RCT (2 groups)
}

Single-blind (outcomes assessor)

Follow-up: 12 months, 24 months

Participants

60 participants

Inclusion criteria

1. Clinical

1. Clinical lacunar stroke syndrome within the past 6 months

2. Absence of signs or symptoms of cortical dysfunction

3. No proximal large vessel atherosclerosis, intracranial atherosclerosis or cerebellar stroke

4. No major cardioembolic source requiring anticoagulation or other specific therapy

2. Imaging

1. MRI presence of a small subcortical ischaemic, any 1 or more of: DWI lesion $<2.0 \mathrm{~cm}$ in size at largest dimension and corresponding to the clinical syndrome. Well delineated focal hyperintensity $<2.0 \mathrm{~cm}$ in size at largest dimension (including rostro-caudal extent) on FLAIR or T2 and clearly corresponding to the clinical syndrome. If other focal hyperintensities are present, the case will be discussed with the principal investigator prior to randomisation. Multiple (at least 2) hypointense lesions of size $0.3-1.5 \mathrm{~cm}$ at largest dimension (including rostro-caudal extent) only in the cerebral hemispheres on FLAIR or T1 in people whose qualifying event is clinically hemispheric. Well delineated hypointense lesion $<1.5 \mathrm{~cm}$ in size at the largest dimension (including rostro-caudal extent) on FLAIR or T1 corresponding to the clinical syndrome. MRI must be done at least 1 month after the qualifying stroke

2. Absence of cortical stroke and large $(>1.5 \mathrm{~cm})$ subcortical stroke, recent or remote

3. White matter hyperintensity score of 2 (moderate) or 3 (severe) on the European Scale of AgeRelated White Matter Change

4. Absence of cerebral amyloid antipathy (CAA) as per Boston Criteria

Exclusion criteria: 
1. Disabling stroke (Rankin Scale $\geq 4$ )

2. Previous intracranial haemorrhage (excluding traumatic) or hemorrhagic stroke

3. Age under 40 years

4. High risk of bleeding (e.g. recurrent GI or GU bleeding, active peptic ulcer disease, etc.)

5. Anticipated requirement for long term use of anticoagulants (e.g. recurrent deep venous thrombosis)

6. Prior cortical or retinal stroke (diagnosed either clinically or by neuroimaging), or other prior cortical or retinal TIA

7. Prior ipsilateral carotid endarterectomy

8. Impaired renal function: estimated GFR $<40$

9. Intolerance or contraindications to aspirin or clopidogrel (including thrombocytopenia, prolonged INR)

10.Mini Mental Status Exam score $<24$ (adjusted for age and education)

11. Medical contraindication to MRI

12.Pregnancy or women of child-bearing age who are not following an effective method of contraception

13.Pre-existing neurologic, psychiatric, or advanced systemic disease that would confound the neurological or functional outcome evaluations

14.SBP $<90$ or $>200$

15. Known history of limb vascular disease, limb vascular bypass surgery, or limb deep venous thrombosis

16.Prisoners

17. Homeless individuals

18. People unable to give informed consent and no available legally authorised representative to provide informed consent

19.People unlikely to be compliant with therapy/unwilling to return for follow-up visits

20.Concurrent participation in another study with investigational drug or device treatment

21.Other likely specific cause of stroke (e.g. dissection, vasculitis, prothrombotic diathesis, drug abuse)

Interventions

1. RIC group: in combination with intensive standard medical secondary prevention stroke treatment, all participants will undergo RIC once or twice daily for up to four 5-minute cycles of bilateral upper extremity ischaemia separated by 5-minute periods of reperfusion

2. Control group: intensive standard medical secondary prevention stroke treatment alone

Outcomes Primary outcome

1. Feasibility (time frame: 12 months): primary endpoints will be descriptive statistics describing the implementation of the RPreC procedure, including behavioral adherence to treatment, physiologic attainment of limb ischaemia, and patient self-reported comfort-discomfort during treatment

Secondary outcomes

1. Safety (time frame: 24 months): the lead safety endpoint will be all serious adverse events, and the secondary safety endpoints will be deep and superficial vein thrombosis and all cause mortality

2. Biomarker efficacy (time frame: 12 months): the lead efficacy endpoint will be volumetric progression of white matter ischaemic injury on magnetic resonance imaging (biomarker efficacy); and the primary MRI endpoint will be the proportion of people with increase in total white matter hyperintensity volume (as $\%$ of total brain volume) above $1.0 \%$

\begin{tabular}{ll}
\hline Starting date & November 2015 \\
\hline Contact information & Latisha K Sharma, MD; Tel: 310-794-6379; email: lali@mednet.ucla.edu \\
\hline
\end{tabular}


NCT02169739 (Continued)

Notes
The study is currently recruiting participants. The estimated study completion date is June 2018. Results not yet published

NCT02189928

\section{Trial name or title}

REmote iSchemic Conditioning in acUtE BRAin INfarction Study (RESCUE-BRAIN)

A multicenter, randomised, controlled study (2 groups)

Single-blind (outcomes assessor)

Follow-up: 24 hours, 7 days, 3 months

\section{Participants}

\section{0 participants}

Inclusion criteria

1. Patients $\geq 18$ years

2. Carotid ischaemic stroke

3. NIHSS score between 5 and 25

4. Brain MRI performed within 6 hours from symptoms onset

5. Obtaining a written informed consent of the patient or a third party (parent or close), or emergency inclusion process

6. Affiliated with a Medicare (or rightful beneficiary)

\section{Exclusion criteria}

1. Presence of a leg ulcer or a bad skin condition in the lower limbs

2. History of arterial occlusive disease of the lower limbs

3. Sickle cell disease known (risk of vaso-occlusive crisis)

4. History of phlebitis in the lower limbs

5. History of cerebral infarction older than 3 months

6. Participation in another interventional acute phase protocol

7. People under guardianship

8. Pathologies involving life-threatening within 6 months and making it impossible to evaluate to 3 months

9. Pople depending on others before the ischaemic stroke (Rankin Score previous $>2$ )

10.Pregnant women
1. RIC group: usual care (thrombolysis or not) with RIC. RIC is performed with lower limb tourniquet put on half thigh, the protocol includes 4 cycles ( 5 minutes each) of inflation cuff $110 \mathrm{mmHg}$ above systolic blood pressure followed by 4 cycles ( 5 minutes each) of deflation in between

2. Control group: usual care (thrombolysis or not)

1. Brain MRI changes of DWI brain infarction volume (cc) between baseline $(<\mathrm{H} 6)$ and day 1 in the 2 groups of participants (time frame: 24 hours)

Secondary outcome

1. Percentage of participants with $\mathrm{mRS}<2$ at 3 months in the 2 groups (time frame: 3 months)

Other outcomes 
NCT02189928 (Continued)

1. Safety of remote perconditioning ischaemic process (time frame: 7 days): rate of lower limb ischaemia and deep venous thrombosis in Per CID group

2. Safety of remote perconditioning ischaemic process (time frame: 7 days): rate of early neurological worsening (NIHSS score (d1- d0) $>4$ points) in Per-CID and control group

3. Safety of remote perconditioning ischaemic process (time frame: 7 days): percentage of hemorrhagic transformation in case of IV thrombolysis in the 2 groups

\begin{tabular}{ll}
\hline Starting date & January 2015 \\
\hline Contact information & Amina Cattenoy, Project Manager; Tel: +33 1392397 77; email: acattenoy@ch-versailles.fr \\
\hline Notes & $\begin{array}{l}\text { The study is currently recruiting participants. The estimated study completion date is January } \\
\text { 2019. Results not yet published }\end{array}$ \\
\hline
\end{tabular}

\section{NCT02323425}

Trial name or title Effects of limb ischaemic postconditioning in young sICAS (EPIC-sICAS)

\begin{tabular}{ll}
\hline Methods & RCT (2 groups) \\
& Open label \\
& Follow-up: 14 days, 1 month, 6 months, and 1 year
\end{tabular}

\begin{tabular}{|c|c|}
\hline \multirow[t]{19}{*}{ Participants } & 100 participants \\
\hline & Inclusion criteria \\
\hline & 1. Age between 18 to 45 years old \\
\hline & $\begin{array}{l}\text { 2. Symptomatic intracranial atherosclerotic stenosis (SICAS): cranial CTA/MRA/TCD/DSA confirmed } \\
\text { the diagnosis, participants with ischaemic stroke or TIA in the brain region supplied by the stenosis } \\
\text { artery }\end{array}$ \\
\hline & 3. NIHSS score $0-15$ \\
\hline & 4. Written informed consent was signed \\
\hline & Exclusion criteria \\
\hline & 1. Cerebral haemorrhage and other parts of the active bleeding disease \\
\hline & 2. Severe aphasia, unable to express himself \\
\hline & 3. History of brain tumour, brain trauma, cerebral embolism or other brain lesions \\
\hline & $\begin{array}{l}\text { 4. Severe lesions of severe cardiac, liver or kidney disease, malignancy or other systemic organ dys- } \\
\text { function }\end{array}$ \\
\hline & 5. Blood pressure $<90 \mathrm{mmHg} / 60 \mathrm{mmHg}$ or $>200 \mathrm{mmHg} / 110 \mathrm{mmHg}$ after treatment \\
\hline & 6. Dementia or mental illness \\
\hline & 7. Using angiotensin-converting enzyme inhibitors \\
\hline & 8. History of major surgery or trauma 4 weeks prior to admission \\
\hline & 9. Without informed consent \\
\hline & Elimination criteria \\
\hline & 1. People with poor compliance, refusal to take regular treatment and examination \\
\hline & 2. Patient's condition became exacerbated, with NIHSS score elevated more than 4 \\
\hline
\end{tabular}

Interventions

1. RIC group: standard medical management combined with RIC twice daily for 180 consecutive days. RIC procedure: bilateral upper arms ischaemia by inflation cuff to $180 \mathrm{mmHg}$ for $3 \times 5 \mathrm{~min}$ utes, interspersed with 5 minutes of no inflation and thus reperfusion 
1. Mean change of collateral circulation within 6 months post treatment (time frame: baseline and 6 months after treatment): collateral circulation will be evaluated by Magnetic Resonance Angiography (MRA), Dynamic contrast-enhanced magnetic resonance imaging (DCE-MRI), Arterial spinlabelled (ASL) perfusion magnetic resonance, transcranial cerebral Doppler (TCD), single photon emission computed tomography (SPECT).

Secondary outcomes

1. Mean change of symptomatic recovery (time frame: baseline and 14 days, 1 month, 6 months, and 1 year after treatment): neurological function will be assessed by the National Institute of Health stroke scale (NIHSS) scores and Activity of Daily Living Scale (ADL)

2. Mean change of serum vascular endothelial growth factor (VEGF) and basic Fibroblast Growth Factor (bFGF) within 10 days after treatment (time frame: baseline and 10 days after treatment)

\begin{tabular}{ll}
\hline Starting date & March 2015 \\
\hline Contact information & Guogang Luo, M.D, Ph.D; Tel: +8618991232449; email: Iguogang@163.com \\
\hline Notes & $\begin{array}{l}\text { The study is currently recruiting participants. The estimated study completion date is March 2018. } \\
\text { Results not yet published }\end{array}$ \\
\hline
\end{tabular}

Trial name or title Remote ischaemic conditioning for avoiding recurrence of symptomatic intracranial atherosclerotic stenosis

A multicentre, randomised, placebo-controlled study (2 groups)
Quadruple-blinded (participant, care provider, investigator, outcomes assessor)
Follow-up: 1 year, 3 years

Participants 3000 participants

Inclusion criteria

1. Men or women age from 40 to 80 years old

2. People having an ischaemic stroke or a TIA prior to randomisation. People having an ischaemic stroke within 30 days with mRS score $\leq 4$ at baseline. People having a TIA within 15 days with Oxfordshire Community Stroke Project on the basis of age, blood pressure (BP), clinical features, and duration of TIA symptoms (ABCD2) score $\geq 4$ at baseline

3. The entry event is attributed to symptomatic atherosclerotic stenosis (50-99\%) in an intracranial qualifying artery (carotid artery, middle cerebral artery (M1), vertebral artery, or basilar artery) that is documented by MRA or CTA

4. Informed consent obtained

Exclusion criteria

1. Thrombolytic therapy within 24 hours prior to enrolment

2. Progressive neurological signs within 24 hours prior to enrolment

3. Cerebral venous thrombosis/stenosis

4. Intracranial arterial stenosis due to arterial dissection, Moya Moya disease; any known vasculitic disease; herpes zoster, varicella zoster or other viral vasculopathy; neurosyphilis; any other intracranial infection; any intracranial stenosis associated with cerebrospinal fluid (CSF) pleocyto- 
sis; radiation induced vasculopathy; fibromuscular dysplasia; sickle cell disease; neurofibromatosis; benign angiopathy of central nervous system; post-partum angiopathy; suspected vasospastic process, suspected recanalized embolus

5. Any of the following unequivocal cardiac source of embolism: rheumatic mitral and or aortic stenosis, prosthetic heart valves, atrial fibrillation, atrial flutter, sick sinus syndrome, left atrial myxoma, patent foramen ovale, left ventricular mural thrombus or valvular vegetation, congestive heart failure, bacterial endocarditis, or any other cardiovascular condition interfering with the participation

6. Uncontrolled severe hypertension (sitting systolic blood pressure $>180 \mathrm{mmHg}$ and/or sitting diastolic blood pressure $>110 \mathrm{mmHg}$ after medication)

7. People with abnormal laboratory parameters: aspartate transaminase (AST) and/or alanine transaminase $(A L T)>3 \times$ upper limit of normal range; creatinine clearance $<0.6 \mathrm{ml} / \mathrm{s}$ and/or serum creatinine $>265 \mu \mathrm{mol} / \mathrm{l}(>3.0 \mathrm{mg} / \mathrm{dl}$ ); platelets $<100 \times 109 / \mathrm{L}$

8. Any intracranial haemorrhage (parenchymal, subarachnoid, subdural, epidural) within 90 days prior to enrolment

9. Intracranial neoplasm, cerebral aneurysm or arteriovenous malformation

10.Known retinal haemorrhage or visceral bleeding within 30 days prior to enrolment

11.Severe haemostatic disorder or severe coagulation dysfunction

12.Subclavian arterial stenosis $\geq 50 \%$ or subclavian steal syndrome

13. Extracranial stenosis $\geq 50 \%$

14.Previous treatment of target lesion with a stent, angioplasty, or other mechanical device, or plan to perform one of these procedures within 12 months after enrolment

15. Major surgery (including open femoral, aortic, or carotid surgery, cardiac) within previous 30 days or scheduled in the 12 months after enrolment

16.Contraindication for RIC: severe soft tissue injury, fracture, or peripheral vascular disease in the upper limbs

17. Life expectancy $<3$ years

18.Pregnant or breastfeeding women

19. Unwilling to be followed-up or poor compliance for treatment

20.People being enrolled or having been enrolled in other clinical trial within 3 months prior to this clinical trial

21.People unsuitable for enrolment in the clinical trial according to investigators decision making

Interventions

1. RIC group: participants will be treated with RIC once daily for 12 months. RIC treatment is comprised of 5 cycles of bilateral upper limb ischaemia and reperfusion, which will be induced by 2 cuffs placed around the upper arms respectively and inflated to $200 \mathrm{mmHg}$ for 5 minutes followed by 5 minutes of reperfusion by cuff deflation

2. Sham-RIC group: participants will be treated with sham-RIC once daily for 12 months. Sham-RIC treatment is comprised of 5 cycles of bilateral upper limb ischaemia and reperfusion, which will be induced by 2 cuffs placed around the upper arms respectively and inflated to $60 \mathrm{mmHg}$ for $5 \mathrm{~min}$ utes followed by 5 minutes of reperfusion by cuff deflation

1. Time from randomisation to the first occurrence of fatal or non-fatal ischaemic stroke (time frame: during the first 12 months from randomisation)

Secondary outcomes

1. Time from randomisation to the first occurrence of a composite of fatal or non-fatal stroke (ischaemic and haemorrhagic), fatal or non-fatal myocardial infarction, and TIA (time frame: during the first 12 months from randomisation)

2. Time from randomisation to the first occurrence of each component of the composite of fatal or non-fatal stroke (ischaemic and haemorrhagic), fatal or non-fatal myocardial infarction, and TIA (time frame: during the first 12 months from randomisation)

3. Time to death from all causes from randomisation (time frame: during the first 12 months from randomisation) 
NCT02534545 (Continued)

Other outcomes

1. Scores assessed by NIHSS (time frame: during the first 12 months from randomisation)

2. Scores assessed by $\mathrm{mRS}$ (time frame: during the first 12 months from randomisation)

3. Scores assessed by BI (time frame: during the first 12 months from randomisation)

4. Number of participants with abnormal laboratory values (time frame: 3 years)

5. Number of participants with adverse events that are related to treatment (time frame: 3 years)

\begin{tabular}{ll}
\hline Starting date & September 2015 \\
\hline Contact information & Xunming Ji, MD. PhD; Tel: 86-10-83198952; email: jixunming@vip.163.com \\
\hline Notes & $\begin{array}{l}\text { The study is currently recruiting participants. The estimated study completion date is September } \\
\text { 2018. Results not yet published }\end{array}$ \\
\hline
\end{tabular}

NCT02779712

\begin{tabular}{ll}
\hline Trial name or title & Remote ischaemic conditioning after stroke trial (ReCAST-2) \\
\hline Methods & RCT (2 groups) \\
& Double-blinded (participant, outcomes assessor) \\
& Follow-up: 1 day, 14 days, 90 days \\
\hline Participants & 60 participants \\
& Inclusion criteria \\
& 1. Suspected clinical stroke with 6 hours of onset of neurological symptoms \\
2. Age $>18$ \\
3. Written or witnessed oral consent, or relative/consulter advice \\
Exclusion criteria \\
1. Pre-morbid dependency mRS $>3$ \\
2. Dementia \\
3. Coma (GCS $<8$ ) \\
4. Malignancy or significant co-morbidity thought to limit life expectancy to $<6$ months \\
5. Blood sugar $<3.5$ mmol/L \\
6. Taking part in another clinical trial of an investigational medicinal product (CTIMP) \\
7. Pregnancy
\end{tabular}

Interventions

1. RIC group: 4 cycles of intermittent limb ischaemia - alternating 5 minutes' inflation $(20 \mathrm{mmHg}$ above systolic BP) followed by 5 minutes' deflation of a standard upper arm blood pressure cuff. 1 dose ( $=4$ cycles) of intermittent upper limb ischaemia ( 1 cycle $=5$ minutes' inflation to $20 \mathrm{mmHg}$ above systolic BP, 5 minutes deflation). Dose escalation: a) recruits 1-20 receive this cycle once; $b$ ) recruits 21-40 receive a second dose of 4-cycles 1 hour after the first; c) recruits 41-60 receive further dosing, twice daily until day 4.

2. Sham-RIC group: 4 cycles of alternating 5 minutes' inflation (up to $30 \mathrm{mmHg}$ ) followed by $5 \mathrm{~min}$ utes' deflation of a standard upper arm blood pressure cuff. 4 cycles of intermittent sham procedure ( 1 cycle $=5$ minutes' inflation to $30 \mathrm{mmHg}, 5$ minutes' deflation), matching the dose escalation described in the intervention 
1. Trial feasibility (time frame: 90 days)

Secondary outcomes

1. Vascular event rate (time frame: day 1 , day $4 \pm 1$, day $90 \pm 7$ ): number of participants with a vascular event (including limb ischaemia, recurrent stroke, myocardial infarction, venous thrombo-embolism)

2. Treatment-related serious adverse event rates (time frame: day 1 , day $4 \pm 1$, day $90 \pm 7$ )

3. Plasma biomarkers (time frame: immediately before RIC/sham at baseline; immediately after RIC/ sham on day 1 ; day $4 \pm 1$ ): biomarkers include S100-beta protein, heat-shock proteins and cytokines

4. Impairment (time frame: day $4 \pm 1$, day $90 \pm 7$ ): assessed by the NIHSS

5. Dependency (time frame: day $90 \pm 7$ ): assessed by the $\mathrm{mRS}$

6. Disability (time frame: day $90 \pm 7$ ): assessed by the $\mathrm{BI}$

7. Mood (time frame: day $90 \pm 7$ ): assessed by Zung Depression Scale

8. Telephone cognition (time frame: day $90 \pm 7$ ): assessed by Modified Telephone Interview for Cognitive Status

\begin{tabular}{ll}
\hline Starting date & August 2016 \\
\hline Contact information & Nikola Sprigg, MD; email: nikola.sprigg@nottingham.ac.uk \\
\hline Notes & The study is currently recruiting participants. The estimated study completion date is September \\
& 2018. Results not yet published \\
\hline
\end{tabular}

\section{NCT03105141}

Trial name or title

Optimized remote ischaemic conditioning (RIC) treatment for patients with chronic cerebral ischaemia

Methods RCT

Double-blinded (care provider, outcomes assessor)

Follow-up: 1, 3, 6, and 12 months

\section{Participants}

600 participants

Inclusion criteria

1. Age ranging from 45 to 80 years of age, both genders

2. Patients diagnosed with an ischaemic stroke or TIA before admission and the following requirements should be satisfied as well: the occurrence of an ischaemic stroke within 30 days and with a baseline mRS score $\leq 4$; the occurrence of a TIA within 15 days and with a baseline Oxfordshire Community Stroke Project on the basis of age, blood pressure, clinical features, and duration of TIA symptoms (ABCD2) score $\geq 4$

3. Patients with symptomatic intracranial atherosclerotic stenosis that is attributed to at least $50 \%$ stenosis of the diameter of a major intracranial artery (carotid artery, or middle cerebral artery (M1)) verified by MRA or CTA

4. Informed consent obtained from patients or health care proxy, as appropriate, able to cooperate and participate in follow-up visits

Exclusion criteria

1. Received intravenous or intraarterial thrombolytic therapy such as rtPA within 24 hours prior to inclusion

2. Progressive deterioration of neurological manifestations within 24 hours prior to inclusion 
3. Intracranial arterial stenosis due to any of the following non-atherosclerotic aetiologies, for instance, Moyamoya disease, artery dissection, any known vasculitic disease, any intracranial infection, radiation induced vasculopathy, cerebrospinal fluid pleocytosis-associated intraarterial stenosis, genetic or developmental abnormalities such as fibromuscular dysplasia, neurofibromatosis, sickle cell disease, and mitochondrial encephalopathy, intracranial granulomatous arteritis, postpartum angiopathy, suspected vasospasm or embolism, iatrogenic trauma

4. Intracranial abnormalities such as tumour, abscess, vascular malformation, cerebral venous sinus stenosis or thrombosis

5. Any haemorrhagic transformation or large area of cerebral infarction (more than $1 / 3$ of MCA perfusion territory)

6. Any type of intracranial haemorrhage within 90 days prior to inclusion

7. Potential cardiac source of embolism such as rheumatic mitral and or aortic stenosis, prosthetic heart valves, atrial flutter, atrial fibrillation, left atrial myxoma, sick sinus syndrome, patent foramen ovale, left ventricular mural thrombus or valvular vegetation, congestive heart failure, and bacterial endocarditis

8. Previous stent, angioplasty, or other mechanical device in the target lesion, or plan to receive any of the above procedures within 12 months after inclusion

9. Refractory hypertension (systolic blood pressure $>180 \mathrm{mmHg}$; diastolic blood pressure $>110$ $\mathrm{mmHg}$ ) that cannot be controlled by medical intervention

10.Above $50 \%$ stenosis of extracranial artery (carotid artery or vertebral artery)

11.Above $50 \%$ stenosis of subclavian artery or subclavian steal syndrome

12. Retinal haemorrhage or visceral bleeding within 30 days prior to inclusion

13. Myocardial infarction within previous 30 days prior to inclusion

14.Previous history of major surgery within 30 days prior to inclusion, or arranged for any of the procedure within 12 months after inclusion

15.Severe haemostatic or coagulation disorders (haemoglobin $<10 \mathrm{~g} / \mathrm{dL}$, blood platelet count $<100$ $\times 109 / \mathrm{L})$

16. Hepatic or renal dysfunctions (aspartate transaminase and/or alanine transaminase $>3 \times$ upper limit of normal range, creatinine clearance $<0.6 \mathrm{ml} / \mathrm{s}$ and/or serum creatinine $>265 \mu \mathrm{mol} / \mathrm{l}(>3.0$ $\mathrm{mg} / \mathrm{dl})$ )

17.Current or having a history of chronic physical diseases or mental disorders

18.Life expectancy $<3$ years due to concomitant life-threatening illness

19. Contraindications for RIC: significant peripheral arterial disease, soft tissue or vascular injury, wounds, and fracture affecting the upper limbs

20.Pregnant or lactating women

21.Patients unlikely to be compliant with intervention or return for follow-up visits

22.No consent obtained from the patient or available legally authorised representatives

23. Patients recruited into another clinical investigation with medication or device, which is likely to impact the outcome of this study

\section{Substudy 1: optimising inflating pressure}

Group 1: participants will be treated with RIC twice daily for 12 months. RIC treatment is comprised of 5 cycles of bilateral upper limb ischaemia and reperfusion, which will be induced by 2 cuffs placed around the upper arms respectively and inflated to $200 \mathrm{mmHg}$ for 5 minutes followed by 5 minutes of reperfusion by cuff deflation.

Group 2: participants will be treated with RIC twice daily for 12 months. RIC treatment is comprised of 5 cycles of bilateral upper limb ischaemia and reperfusion, which will be induced by 2 cuffs placed around the upper arms respectively and inflated to $40 \mathrm{mmHg}$ above systolic pressure for 5 minutes followed by 5 minutes of reperfusion by cuff deflation.

\section{Substudy 2: optimising cycles for application}

Group 1: participants will be treated with RIC twice daily for 12 months. RIC treatment is comprised of 4 cycles of bilateral upper limb ischaemia and reperfusion, which will be induced by 2 cuffs placed around the upper arms respectively and inflated to $200 \mathrm{mmHg}$ for 5 minutes followed by 5 minutes of reperfusion by cuff deflation. 
Group 2: participants will be treated with RIC twice daily for 12 months. RIC treatment is comprised of 5 cycles of bilateral upper limb ischaemia and reperfusion, which will be induced by 2 cuffs placed around the upper arms respectively and inflated to $200 \mathrm{mmHg}$ for 5 minutes followed by 5 minutes of reperfusion by cuff deflation.

Group 3: participants will be treated with RIC twice daily for 12 months. RIC treatment is comprised of 6 cycles of bilateral upper limb ischaemia and reperfusion, which will be induced by 2 cuffs placed around the upper arms respectively and inflated to $200 \mathrm{mmHg}$ for 5 minutes followed by 5 minutes of reperfusion by cuff deflation.

\section{Substudy 3: optimising ischaemia duration}

Group 1: participants will be treated with RIC twice daily for 12 months. RIC treatment is comprised of 5 cycles of bilateral upper limb ischaemia and reperfusion, which will be induced by 2 cuffs placed around the upper arms respectively and inflated to $200 \mathrm{mmHg}$ for 5 minutes followed by 4 minutes of reperfusion by cuff deflation.

Group 2: participants will be treated with RIC twice daily for 12 months. RIC treatment is comprised of 5 cycles of bilateral upper limb ischaemia and reperfusion, which will be induced by 2 cuffs placed around the upper arms respectively and inflated to $200 \mathrm{mmHg}$ for 5 minutes followed by 5 minutes of reperfusion by cuff deflation.

Group 3: participants will be treated with RIC twice daily for 12 months. RIC treatment is comprised of 5 cycles of bilateral upper limb ischaemia and reperfusion, which will be induced by 2 cuffs placed around the upper arms respectively and inflated to $200 \mathrm{mmHg}$ for 5 minutes followed by 6 minutes of reperfusion by cuff deflation.

\section{Substudy 4: optimising times daily}

Group 1: participants will be treated with RIC twice daily for 12 months. RIC treatment is comprised of 5 cycles of bilateral upper limb ischaemia and reperfusion, which will be induced by 2 cuffs placed around the upper arms respectively and inflated to $200 \mathrm{mmHg}$ for 5 minutes followed by 5 minutes of reperfusion by cuff deflation.

Group 2: participants will be treated with RIC once daily for 12 months. RIC treatment is comprised of 5 cycles of bilateral upper limb ischaemia and reperfusion, which will be induced by 2 cuffs placed around the upper arms respectively and inflated to $200 \mathrm{mmHg}$ for 5 minutes followed by 5 minutes of reperfusion by cuff deflation.

1. Recurrent rate of ischaemic stroke and/or TIA (time frame: within 12 months)

Secondary outcomes

1. Treatment of related adverse events (time frame: within 12 months)

2. The incidence of composite vascular events (time frame: within 12 months)

3. The incidence of each component of composite vascular events (time frame: within 12 month)

4. The incidence of all cause mortality (time frame: within 12 months)

5. The evaluation of cognitive function (time frame: baseline, within 3, 6 and 12 months)

6. The evaluation of neurological impairment caused by a stroke (time frame: baseline, within 3, 6 and 12 months)

7. The evaluation of degree of disability or dependence in the daily activities (time frame: baseline, within 3,6 and 12 months)

8. The evaluation of performance in activities of daily living (time frame: baseline, within 3,6 and 12 months)

9. Mean changes in cerebral blood perfusion and/or collateral circulation (time frame: baseline, within 12 months)

10. Mean change in brain function (time frame: baseline, within 12 months)

11.Changes in cerebral white matter lesions (WMLs) (time frame: baseline, within 12 months)

12. The occurrence of additional neurological events (time frame: within 12 months) 
NCT03105141 (Continued)

13. The evaluation of serum biomarkers (time frame: baseline, within 1, 3, 6 and 12 months

Starting date 1 June 2017

Contact information

Ran Meng, MD, PhD; +86-10-83198952; Ranmeng2011@pku.org.cn

Da Zhou, MD, PhD candidate; +86-10-83198952; popdoctor@foxmail.com

Notes

The study is currently recruiting participants. The estimated study completion date is January

2020. Results not yet published

NCT03208166

Trial name or title

Pilot study of pre-ischaemic conditioning for intracranial atherosclerosis (PICASSO)

\begin{tabular}{ll}
\hline Methods & RCT (2 groups) \\
& Open label \\
Follow-up: 30 days
\end{tabular}

Participants

10 participants

Inclusion criteria

1. Symptomatic cerebral infarction within 30 days of enrolment attributed to $70 \%$ to $99 \%$ stenosis of a major intracranial artery (carotid artery, MCA stem (M1), vertebral artery, or basilar artery) that is documented by any of the following: MRA, CTA, or catheter angiography. Percent stenosis will be measured according to WASID criteria (= 1 - (Ds / Dn)) x 100\% with Ds (diameter of stenosis) and Dn (diameter of normal vessel))

2. Modified Rankin score of $\leq 3$

3. Age $\geq 30$ years and $\leq 90$ years. Participants of $30-49$ years are required to meet at least one additional criteria. This additional requirement is to increase the likelihood that the symptomatic intracranial stenosis in participants of 30-49 years is atherosclerotic: a) insulin dependent diabetes for at least 15 years; b) at least 2 of the following atherosclerotic risk factors: hypertension (BP $>140 / 90$ or on antihypertensive therapy); dyslipidaemia (LDL $>130 \mathrm{mg} / \mathrm{dl}$ or $\mathrm{HDL}<40 \mathrm{mg} / \mathrm{dl}$ or fasting triglycerides $>150 \mathrm{mg} / \mathrm{dl}$ or on lipid lowering therapy); smoking; non-insulin dependent diabetes or insulin dependent diabetes of less than 15 years duration; family history of any of the following: myocardial infarction, coronary artery bypass, coronary angioplasty or stenting, stroke, carotid endarterectomy or stenting, peripheral vascular surgery in parent or sibling who was < 55 years of age for men or $<65$ for women at the time of the event; c) history of any of the following: myocardial infarction, coronary artery bypass, coronary angioplasty or stenting, carotid endarterectomy or stenting, or peripheral vascular surgery for atherosclerotic disease; $d$ ) any stenosis of an extracranial carotid or vertebral artery, another intracranial artery, subclavian artery, coronary artery, iliac or femoral artery, other lower or upper extremity artery, mesenteric artery, or renal artery that was documented by non-invasive vascular imaging or catheter angiography and is considered atherosclerotic; e) aortic arch atheroma documented by non-invasive vascular imaging or catheter angiography; f) any aortic aneurysm documented by non-invasive vascular imaging or catheter angiography that is considered atherosclerotic

4. Recent (within 30 days) negative pregnancy test in a woman who has had any menses in the last 12 months

5. Participant is willing and able to return in 30 days for close-out visit for the study

6. Participant is available by phone

7. Participant is able to apply the conditioning device or has access to another person (family member, friend) who can assist with application of conditioning device if needed

8. Participant understands the purpose and requirements of the study, can make him/herself understood, and has provided informed consent 


\section{Participant is able to undergo brain MRI}

Exclusion criteria

1. Previous treatment of target lesion with a stent, angioplasty, or other mechanical device, or plan to perform one of these procedures

2. Plan to perform concomitant angioplasty or stenting of an extracranial vessel tandem to an intracranial stenosis

3. Intracranial tumour (except meningioma) or any intracranial vascular malformation

4. Thrombolytic therapy within 24 hours prior to enrolment

5. Progressive neurological signs within 24 hours prior to enrolment

6. Any intracranial haemorrhage (parenchymal, subarachnoid, subdural, epidural) within 90 days

7. Any untreated chronic subdural haematoma

8. Intracranial arterial stenosis due to arterial dissection, Moya Moya disease; any known vasculitic disease; herpes zoster, varicella zoster or other viral vasculopathy; neurosyphilis; any other intracranial infection; any intracranial stenosis associated with CSF pleocytosis; radiation induced vasculopathy; fibromuscular dysplasia; sickle cell disease; neurofibromatosis; benign angiopathy of central nervous system; post-partum angiopathy; suspected vasospastic process, suspected recanalised embolus

9. Presence of any of the following unequivocal cardiac sources of embolism: chronic or paroxysmal atrial fibrillation, mitral stenosis, mechanical valve, endocarditis, intracardiac clot or vegetation, myocardial infarction within three months, dilated cardiomyopathy, left atrial spontaneous echo contrast, ejection fraction less than $30 \%$

10. History of upper extremity ischaemia, known subclavian or brachial artery stenosis, subclavian steal syndrome, any upper extremity soft tissue, orthopedic or vascular injury, or mastectomy or other procedure that may contraindicate taking blood pressure or having a cuff on the arm for the conditioning treatment

11.Difference in systolic blood pressure of $>15 \mathrm{mmHg}$ between both arms

12.Known allergy to aspirin or clopidogrel

13.Active peptic ulcer disease, major systemic haemorrhage within 30 days, active bleeding diathesis, platelets $<100,000$, hematocrit $<30$, INR $>1.5$, clotting factor abnormality that increases the risk of bleeding, current alcohol or substance abuse, uncontrolled severe hypertension (systolic pressure $>180 \mathrm{mmHg}$ or diastolic pressure $>115 \mathrm{mmHg}$ ), severe liver impairment (AST or ALT > 3 $\mathrm{x}$ normal, cirrhosis), subject on dialysis

14. Major surgery (including open femoral, aortic, or carotid surgery, cardiac) within previous 30 days or planned in the next 30 days after enrolment

15.Indication for warfarin or heparin beyond enrolment (note: exceptions allowed for subcutaneous heparin for deep vein thrombosis prophylaxis while hospitalised)

16.Diabetics taking sulfonylurea drugs

17.Severe neurological deficit that renders the person incapable of living independently

18.Dementia or psychiatric problem that prevents the person from following the protocol reliably

19. Co-morbid conditions that may limit survival to $<3$ months

20.Pregnancy or of childbearing potential and unwilling to use contraception for the duration of this study

21.Claustrophobia requiring sedation for MRI

22.Enrollment in another study that would conflict with the current study

Interventions

RIC group: RIC and standard medical care. RIC consist of 4 cycles of 5-minute inflations of both blood pressure cuffs simultaneously to a pressure of $200 \mathrm{mmHg}$ with 5 minutes of reperfusion between each inflation.

Control group: standard medical care alone

\section{Outcomes}

Primary outcome: cerebral blood flow (time frame: baseline and 30 days) 
NCT03208166 (Continued)

Trial name or title

Methods
Rt-PA thrombolytic therapy in combination with remote ischaemic conditioning for acute ischaemic stroke

RCT (2 groups)

Single-blind (outcomes assessor)

Follow-up: 7 days, 90 days

\section{0 participants}

Inclusion criteria

1. Male or female, age $\geq 18$

2. Acute ischaemic stroke

3. modified Rankin Scale (mRS) score of $2-5$

4. mRS score less than 1 before onset of stroke symptom

5. Onset of stroke symptoms within $4.5 \mathrm{~h}$ before initiation of intravenous rt-PA thrombolytic therapy

6. Good compliance for RIC therapy

7. Informed consent obtained

Exclusion criteria

1. Stroke or serious head trauma within the previous 3 months

2. Major surgery or severe trauma with in the preceding 3 months

3. Intracranial haemorrhage

4. Systolic pressure greater than $185 \mathrm{mmHg}$ or diastolic pressure greater than $110 \mathrm{mmHg}$, or aggressive treatment intravenous medication necessary to reduce blood pressure to these limits

5. Symptoms rapidly improving

6. Symptoms suggestive of subarachnoid haemorrhage, even if CT scan was normal

7. Gastrointestinal haemorrhage or urinary tract haemorrhage within the previous 21 days

8. Arterial puncture at a noncompressible site within the previous 7 days

9. Seizure at the onset of stroke

10. Platelet count of less than 100,000 per cubic millimetre

11. Received heparin within the 48 hours preceding the onset of stroke and had an elevated partial-thromboplastin time

12. Received oral anticoagulation therapy preceding the onset of stroke and INR greater than 1.7 or prothrombin times greater than 15 seconds

13.CT showed a multiple infarction (low density area greater than $1 / 3$ cerebral hemisphere)

14.Use or plan to use intervention for diagnosis or treating

15. Intracranial neoplasm, cerebral aneurysm or arteriovenous malformation

16.Severe hepatic or renal dysfunction

17.Contraindication for RIC: severe soft tissue injury, fracture, or peripheral vascular disease in the upper limbs

18. Life expectancy $<1$ year

19.Pregnant or breastfeeding women

20.Unwilling to be followed up or poor compliance for treatment 
NCT03231384 (Continued)

21.Patients being enrolled or having been enrolled

Interventions

1. RIC group: RIC will be performed twice daily for 7 consecutive days. The upper limb ischaemic conditioning is composed of 5 cycles of upper limb ischaemia intervened by reperfusion, which is induced by 2 cuffs placed on bilateral arms respectively and inflated to $200 \mathrm{mmHg}$ for 5 minutes followed by 5 minutes of reperfusion by cuff deflation. RIC starts within 2 hours after intravenous thrombolysis. All participants receive a standard clinical management.

2. Control group: all participants received intravenous thrombolysis and standard clinical management.

Outcomes
$\begin{aligned} & \text { Primary outcome } \\ & \text { enrolled participants that completed all the designed RIC procedures }\end{aligned}$
Secondary outcome
$\begin{aligned} & \text { 1. Objective signs of tissue or neurovascular injury ( time frame: within } 7( \pm 1 \text { ) days): palpation of } \\ & \text { distal radial pulses, visual inspection (for local oedema, redness, skin lesion), and palpation for } \\ & \text { tenderness } \\ & \text { 2. Number of participants suffering from intracranial haemorrhage (time frame: } 7 \text { ( } \pm 1 \text { ) days) } \\ & \text { 3. Scores assessed by National Institutes of Health Stroke Scale (NIHSS) (time frame: } 7 \text { ( } \pm 1 \text { ) days) } \\ & \text { 4. The functional outcome of all participants (time frame: } 90 \text { ( } \pm 7 \text { ) days): functional outcomes will } \\ & \text { be assessed by the mRS and BI } \\ & \text { 5. Cerebral infarct volume (time frame: } 3-7 \text { days): the infarct volume will be calculated on MRI and } \\ & \text { CT imaging } \\ & \text { 6. Blood pressure and heart rate during } 7 \text { days of RIC treatment (time frame: } 7 \text { days) } \\ & \text { 7. Plasma biomarkers (time frame: } 7 \text { ( } \pm 2 \text { ) days): including myoglobin, IL-6, HS-CRP and HCY } \\ & \text { 8. Any adverse events (time frame: } 90 \text { ( } \pm 7 \text { ) days) }\end{aligned}$

\begin{tabular}{ll}
\hline Starting date & 30 July 302017 \\
\hline Contact information & Xunming Ji, MD, PhD; Tel: 86-10-83198952; email:jixm@ccmu.edu.cn \\
\hline Notes & $\begin{array}{l}\text { The study is currently recruiting participants. The estimated study completion date is } 31 \text { January } \\
\text { 2018. Results not yet published }\end{array}$ \\
\hline
\end{tabular}

ADL: activities of daily living

ASL: arterial spin-labelled perfusion magnetic resonance

BI: Barthel Index

CTA: computed tomography angiography

DCE-MRI: dynamic contrast-enhanced magnetic resonance imaging

DWI: diffusion weighted imaging

ITT: intention-to-treat

MoCA: Montreal Cognitive Assessment

MMSE: Mini Mental State Examination

MRA: magnetic resonance angiography

$M R I$ : magnetic resonance imaging

mRS: modified Rankin Scale

NIHSS: National Institutes of Health Stroke Scale

PP: per protocol

RCT: randomised controlled trial

RIC: remote ischaemic conditioning

rtPA: recombinant tissue plasminogen activator

SPECT: single-photon emission computed tomography

TCD: transcranial Doppler

TIA: transient ischaemic attack

WMHs: white matter hyperintensities 
DATA AND ANALYSES

\section{Comparison 1. RIC versus non-RIC for preventing ischaemic stroke}

\begin{tabular}{|c|c|c|c|c|}
\hline Outcome or subgroup title & No. of studies & $\begin{array}{l}\text { No. of partici- } \\
\text { pants }\end{array}$ & Statistical method & Effect size \\
\hline $\begin{array}{l}1 \text { Ischaemic stroke or recurrent } \\
\text { ischaemic stroke at completion } \\
\text { of follow-up }\end{array}$ & 3 & 371 & Risk Ratio (M-H, Random, 95\% Cl) & $0.31[0.12,0.76]$ \\
\hline 1.1 Ischaemic stroke & 1 & 189 & Risk Ratio (M-H, Random, 95\% Cl) & $0.22[0.01,4.03]$ \\
\hline 1.2 Recurrent ischaemic stroke & 2 & 182 & Risk Ratio (M-H, Random, 95\% Cl) & $0.32[0.12,0.83]$ \\
\hline $\begin{array}{l}2 \text { Death or dependency at com- } \\
\text { pletion of follow-up }\end{array}$ & 2 & 292 & Risk Ratio (M-H, Random, 95\% Cl) & $0.0[0.0,0.0]$ \\
\hline 2.1 Death & 2 & 292 & Risk Ratio (M-H, Random, 95\% Cl) & $0.0[0.0,0.0]$ \\
\hline 2.2 Dependency & 0 & 0 & Risk Ratio (M-H, Random, 95\% Cl) & $0.0[0.0,0.0]$ \\
\hline 3 Stroke severity & 1 & 189 & $\begin{array}{l}\text { Mean Difference (IV, Random, } \\
95 \% \mathrm{CI})\end{array}$ & $-0.17[-0.23,-0.11]$ \\
\hline 3.1 RIC vs sham control & 1 & 94 & $\begin{array}{l}\text { Mean Difference (IV, Random, } \\
95 \% \mathrm{CI})\end{array}$ & $-0.15[-0.22,-0.08]$ \\
\hline 3.2 RIC vs blank control & 1 & 95 & $\begin{array}{l}\text { Mean Difference (IV, Random, } \\
95 \% \mathrm{CI})\end{array}$ & $-0.21[-0.30,-0.12]$ \\
\hline $\begin{array}{l}4 \text { Improvement in neurological } \\
\text { impairment }\end{array}$ & 0 & & $\begin{array}{l}\text { Mean Difference (IV, Random, } \\
95 \% \mathrm{CI})\end{array}$ & Totals not selected \\
\hline $\begin{array}{l}5 \text { Improvement in psychologi- } \\
\text { cal and cognitive impairment at } \\
\text { completion of follow-up }\end{array}$ & 0 & & $\begin{array}{l}\text { Mean Difference (IV, Random, } \\
95 \% \mathrm{CI})\end{array}$ & Totals not selected \\
\hline 6 Cardiovascular events & 2 & 292 & Risk Ratio (M-H, Random, 95\% Cl) & $0.0[0.0,0.0]$ \\
\hline 6.1 RIC vs sham control & 1 & 94 & Risk Ratio (M-H, Random, 95\% Cl) & $0.0[0.0,0.0]$ \\
\hline 6.2 RIC vs blank control & 2 & 198 & Risk Ratio (M-H, Random, 95\% Cl) & $0.0[0.0,0.0]$ \\
\hline 7 Haemorrhagic stroke & 0 & & Risk Ratio (M-H, Random, 95\% Cl) & Totals not selected \\
\hline $\begin{array}{l}8 \text { Adverse events associated } \\
\text { with RIC treatment }\end{array}$ & 3 & 371 & Risk Ratio (M-H, Random, 95\% Cl) & $10.91[2.01,59.28]$ \\
\hline 8.1 RIC vs sham control & 2 & 173 & Risk Ratio (M-H, Random, 95\% Cl) & $9.78[1.23,77.71]$ \\
\hline 8.2 RIC vs blank control & 2 & 198 & Risk Ratio (M-H, Random, 95\% Cl) & $13.58[0.72,255.07]$ \\
\hline
\end{tabular}


Analysis 1.1. Comparison 1 RIC versus non-RIC for preventing ischaemic stroke, Outcome 1 Ischaemic stroke or recurrent ischaemic stroke at completion of follow-up.

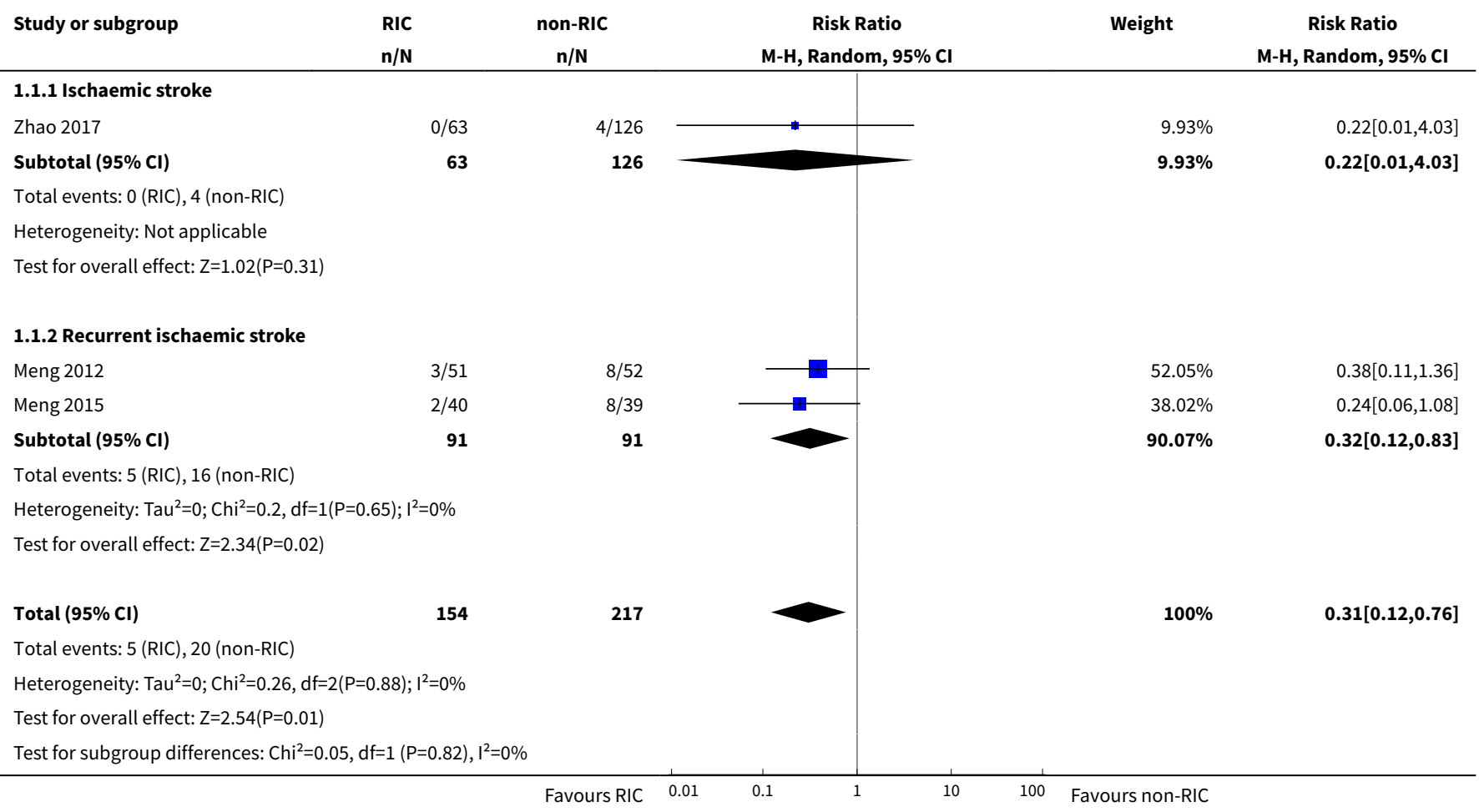

Analysis 1.2. Comparison 1 RIC versus non-RIC for preventing ischaemic stroke, Outcome 2 Death or dependency at completion of follow-up.

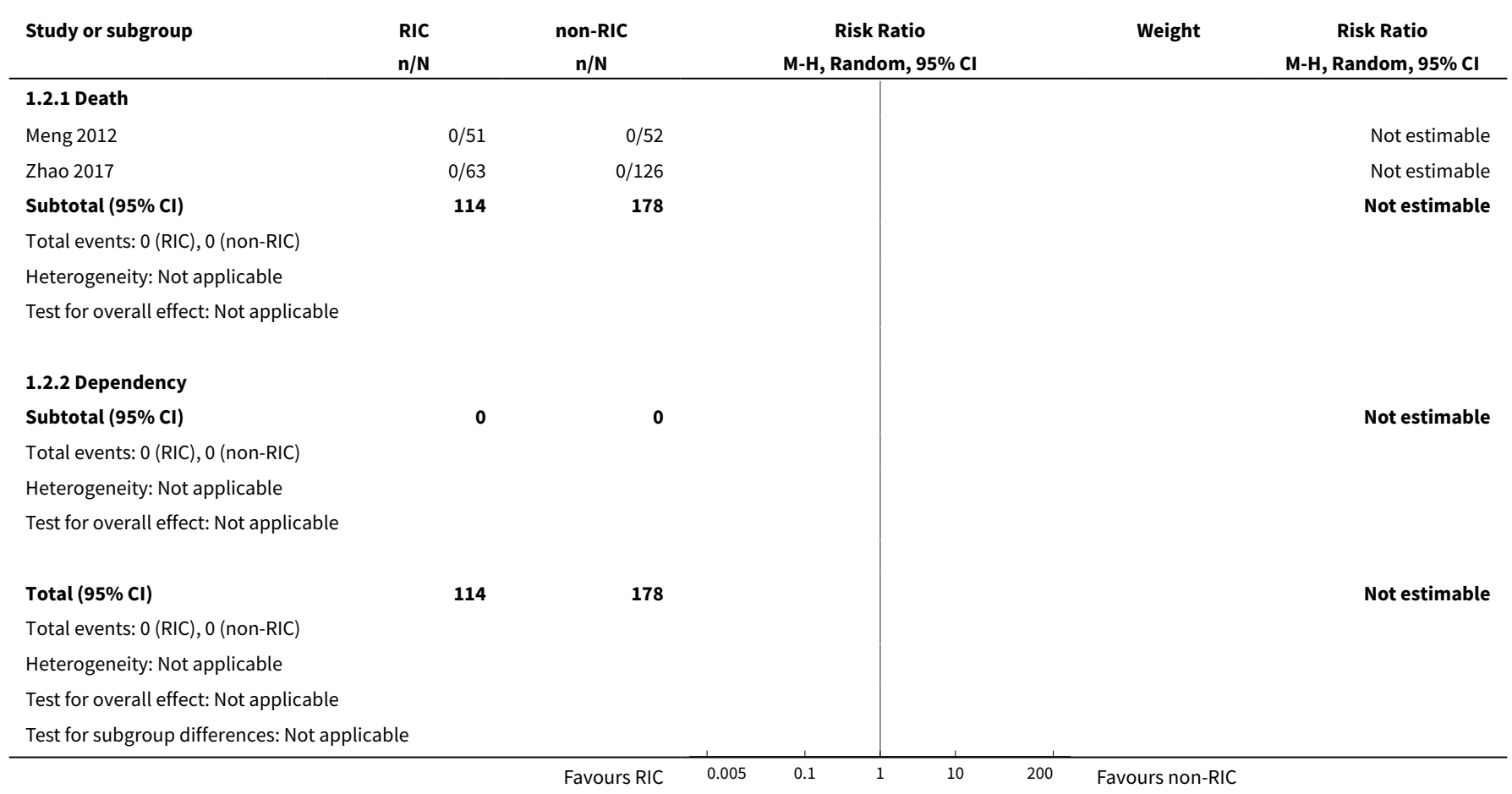


Analysis 1.3. Comparison 1 RIC versus non-RIC for preventing ischaemic stroke, Outcome 3 Stroke severity.

\begin{tabular}{|c|c|c|c|c|c|c|c|}
\hline \multirow[t]{2}{*}{ Study or subgroup } & \multicolumn{2}{|c|}{ Favours RIC } & \multicolumn{2}{|c|}{ non-RIC } & \multirow{2}{*}{$\begin{array}{l}\text { Mean Difference } \\
\text { Random, } 95 \% \mathrm{Cl}\end{array}$} & \multirow[t]{2}{*}{ Weight } & \multirow{2}{*}{$\begin{array}{l}\text { Mean Difference } \\
\text { Random, } 95 \% \mathrm{Cl}\end{array}$} \\
\hline & $\mathbf{N}$ & $\operatorname{Mean}(\mathrm{SD})$ & $\mathbf{N}$ & $\operatorname{Mean}(\mathrm{SD})$ & & & \\
\hline \multicolumn{8}{|c|}{ 1.3.1 RIC vs sham control } \\
\hline Zhao 2017 & 31 & $0.1(0.1)$ & 63 & $0.2(0.3)$ & 1 & $61.88 \%$ & $-0.15[-0.22,-0.08]$ \\
\hline Subtotal $\star \star \star *$ & 31 & & 63 & & & $61.88 \%$ & $-0.15[-0.22,-0.08]$ \\
\hline \multicolumn{8}{|c|}{ Heterogeneity: Not applicable } \\
\hline \multicolumn{8}{|c|}{ Test for overall effect: $Z=4.05(P<0.0001)$} \\
\hline \multicolumn{8}{|c|}{ 1.3.2 RIC vs blank control } \\
\hline Zhao 2017 & 32 & $0.1(0.1)$ & 63 & $0.3(0.4)$ & 14 & $38.12 \%$ & $-0.21[-0.3,-0.12]$ \\
\hline Subtotal $\star \star \star ~$ & 32 & & 63 & & & $38.12 \%$ & $-0.21[-0.3,-0.12]$ \\
\hline \multicolumn{8}{|c|}{ Heterogeneity: Not applicable } \\
\hline \multicolumn{8}{|c|}{ Test for overall effect: $Z=4.44(P<0.0001)$} \\
\hline 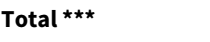 & 63 & & 126 & & & $100 \%$ & $-0.17[-0.23,-0.11]$ \\
\hline \multicolumn{8}{|c|}{ Heterogeneity: $\operatorname{Tau}^{2}=0 ; \mathrm{Chi}^{2}=1.04, \mathrm{df}=1(\mathrm{P}=0.31) ; \mathrm{I}^{2}=3.43 \%$} \\
\hline \multicolumn{8}{|c|}{ Test for overall effect: $Z=5.81(P<0.0001)$} \\
\hline \multicolumn{8}{|c|}{ Test for subgroup differences: $\mathrm{Chi}^{2}=1.04, \mathrm{df}=1(\mathrm{P}=0.31), \mathrm{I}^{2}=3.43 \%$} \\
\hline
\end{tabular}

Analysis 1.6. Comparison 1 RIC versus non-RIC for preventing ischaemic stroke, Outcome 6 Cardiovascular events.

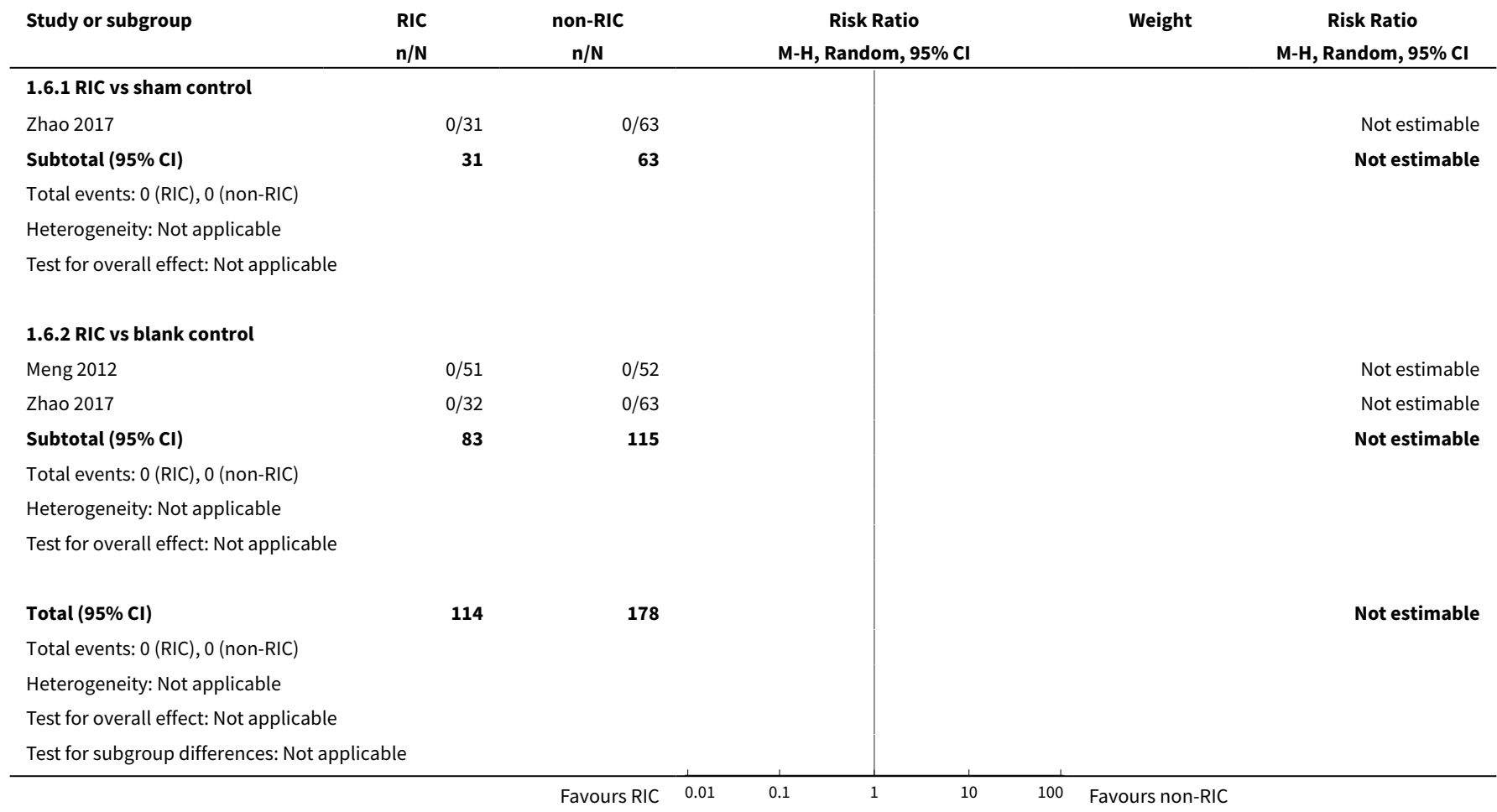


Analysis 1.8. Comparison 1 RIC versus non-RIC for preventing ischaemic stroke, Outcome 8 Adverse events associated with RIC treatment.

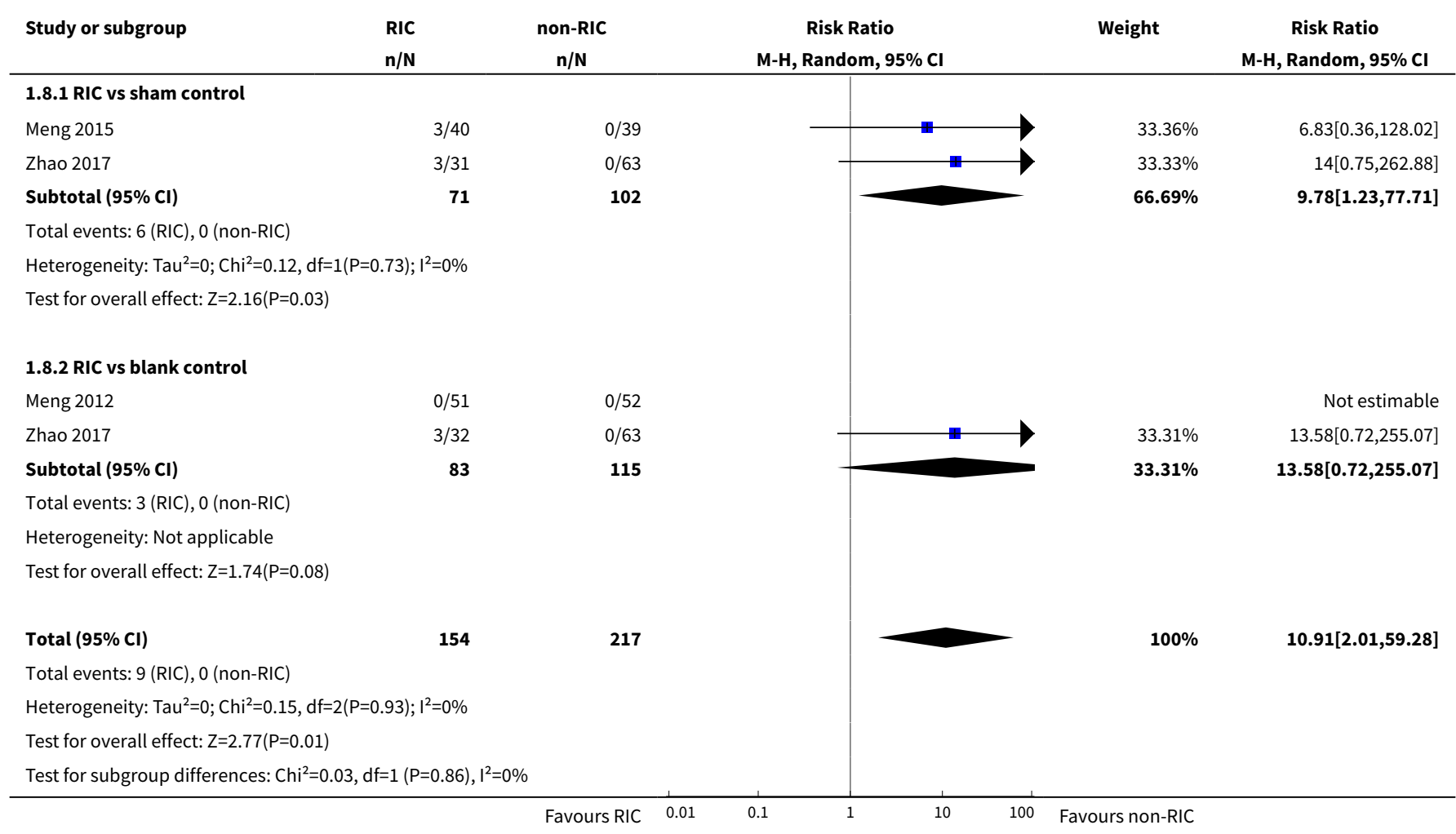

\section{Comparison 2. RIC versus non-RIC treating ischaemic stroke}

\begin{tabular}{lllll}
\hline Outcome or subgroup title & No. of studies & $\begin{array}{l}\text { No. of partici- } \\
\text { pants }\end{array}$ & Statistical method & Effect size \\
\hline $\begin{array}{l}1 \text { Ischaemic stroke or recurrent } \\
\text { ischaemic stroke at completion } \\
\text { of follow-up }\end{array}$ & 0 & & $\begin{array}{l}\text { Risk Ratio (M-H, Random, 95\% } \\
\text { CI) }\end{array}$ & Totals not selected \\
\hline $\begin{array}{l}\text { 2 Death or dependency at the } \\
\text { completion of follow up }\end{array}$ & 1 & & $\begin{array}{l}\text { Risk Ratio (M-H, Random, 95\% } \\
\text { Cl) }\end{array}$ & Totals not selected \\
\hline $\begin{array}{l}3 \text { Stroke severity } \\
\text { 3.1 NIHSS scores }\end{array}$ & 2 & 175 & $\begin{array}{l}\text { Std. Mean Difference (IV, Ran- } \\
\text { dom, 95\% Cl) }\end{array}$ & -0.24 [-1.02, 0.54] \\
\hline $\begin{array}{llll}\text { 3.2 Infarct volume } \\
\text { impairment }\end{array}$ & 1 & 26 & $\begin{array}{l}\text { Std. Mean Difference (IV, Ran- } \\
\text { dom, 95\% Cl) }\end{array}$ & -0.73 [-1.53, 0.07] \\
\hline
\end{tabular}




\begin{tabular}{|c|c|c|c|c|}
\hline Outcome or subgroup title & No. of studies & $\begin{array}{l}\text { No. of partici- } \\
\text { pants }\end{array}$ & Statistical method & Effect size \\
\hline $\begin{array}{l}5 \text { Improvement in psychologi- } \\
\text { cal and cognitive impairment at } \\
\text { completion of follow-up }\end{array}$ & 3 & 105 & $\begin{array}{l}\text { Std. Mean Difference (IV, Fixed, } \\
95 \% \mathrm{CI})\end{array}$ & $-0.29[-0.68,0.11]$ \\
\hline 5.1 Psychological impairment & 1 & 26 & $\begin{array}{l}\text { Std. Mean Difference (IV, Fixed, } \\
95 \% \mathrm{Cl} \text { ) }\end{array}$ & $-0.37[-1.15,0.41]$ \\
\hline 5.2 Cognitive impairment & 3 & 79 & $\begin{array}{l}\text { Std. Mean Difference (IV, Fixed, } \\
95 \% \mathrm{CI} \text { ) }\end{array}$ & $-0.26[-0.72,0.21]$ \\
\hline 6 Cardiovascular events & 0 & & $\begin{array}{l}\text { Risk Ratio (M-H, Random, 95\% } \\
\mathrm{Cl} \text { ) }\end{array}$ & Totals not selected \\
\hline 7 Hemorrhagic stroke & 0 & & $\begin{array}{l}\text { Risk Ratio (M-H, Random, 95\% } \\
\mathrm{Cl} \text { ) }\end{array}$ & Totals not selected \\
\hline $\begin{array}{l}8 \text { Adverse events associated with } \\
\text { RIC treatment }\end{array}$ & 0 & & $\begin{array}{l}\text { Risk Ratio (M-H, Random, 95\% } \\
\mathrm{Cl} \text { ) }\end{array}$ & Totals not selected \\
\hline
\end{tabular}

Analysis 2.2. Comparison 2 RIC versus non-RIC treating ischaemic stroke, Outcome 2 Death or dependency at the completion of follow up.

\begin{tabular}{|c|c|c|c|c|c|c|}
\hline Study or subgroup & $\begin{array}{l}\text { RIC } \\
\mathrm{n} / \mathrm{N}\end{array}$ & $\begin{array}{c}\text { non-RIC } \\
\text { n/N }\end{array}$ & & & $\begin{array}{l}\text { Ratio } \\
\text { om, 95\% Cl }\end{array}$ & $\begin{array}{c}\text { Risk Ratio } \\
\text { M-H, Random, 95\% Cl }\end{array}$ \\
\hline Hougaard 2014 & $30 / 160$ & $10 / 125$ & & & 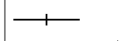 & $2.34[1.19,4.61]$ \\
\hline
\end{tabular}

Analysis 2.3. Comparison 2 RIC versus non-RIC treating ischaemic stroke, Outcome 3 Stroke severity.

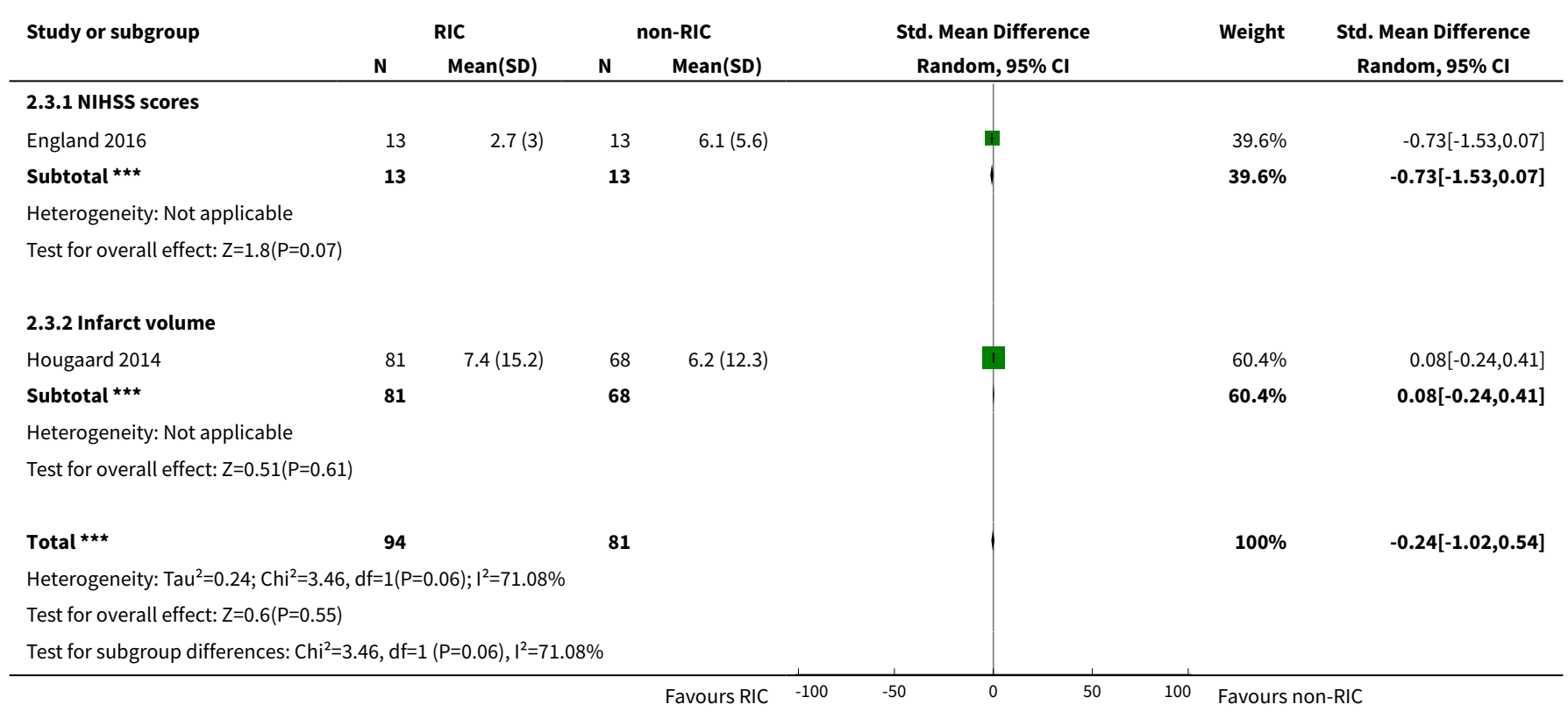


Analysis 2.5. Comparison 2 RIC versus non-RIC treating ischaemic stroke, Outcome 5 Improvement in psychological and cognitive impairment at completion of follow-up.

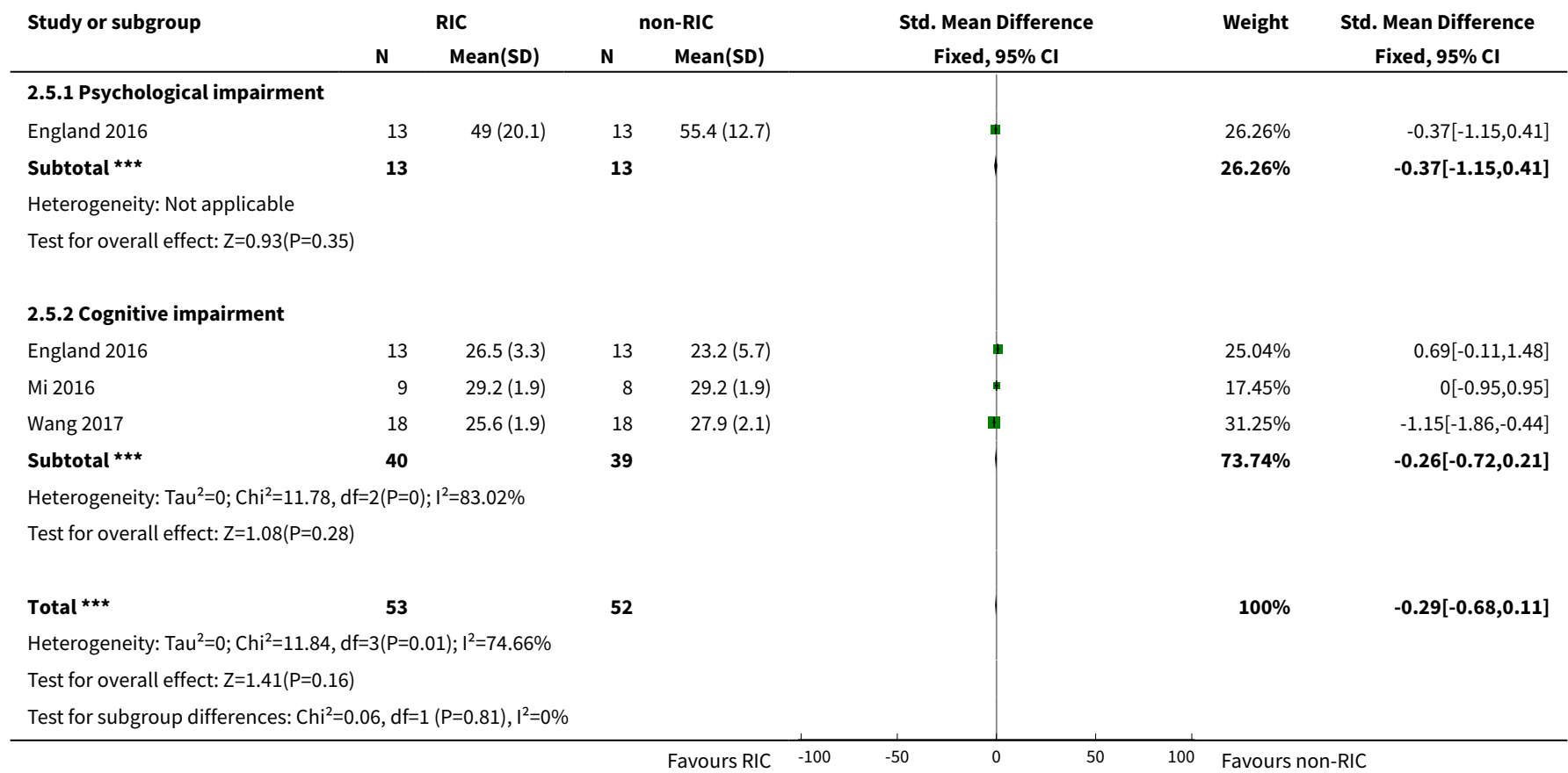

\section{APPEN DICES}

\section{Appendix 1. CENTRAL (the Cochrane Library) search strategy}

\#1[mh ^"brain ischemia"] or [mh "brain infarction"] or [mh ^"carotid artery diseases"] or [mh ^"carotid artery thrombosis"] or [mh $\wedge^{\wedge}$ "carotid artery, internal, dissection"] or [mh $\wedge^{\wedge}$ intracranial arterial diseases"] or [mh ${ }^{\wedge}$ "cerebral arterial diseases"] or [mh ${ }^{\wedge}$ "infarction, anterior cerebral artery"] or [mh ${ }^{\wedge}$ "infarction, middle cerebral artery"] or [mh ^"infarction, posterior cerebral artery"] or [mh "intracranial embolism and thrombosis"] or [mh stroke] or [mh^"basal ganglia cerebrovascular disease"] or [mh ^"vertebral artery dissection"] \#2isch*mi near/6 (stroke* or apoplex ${ }^{\star}$ or cerebral next vasc ${ }^{\star}$ or cerebrovasc* or cva or attack ${ }^{\star}$ ):ti,ab,kw (Word variations have been searched)

\#3(brain or cerebr* or cerebell* or vertebrobasil* or hemispher ${ }^{\star}$ or intracran* or intracerebral or infratentorial or supratentorial or middle next cerebr ${ }^{\star}$ or mca* or "anterior circulation" or "basilar artery" or "vertebral artery") near/5 (stroke* or isch* mi* or infarct* or thrombo* or emboli* or occlus* or hypoxi*):ti,ab,kw (Word variations have been searched)

$\# 4 \# 1$ or \#2 or \#3

\#5[mh ${ }^{\wedge}$ "ischemic postconditioning"] or [mh ${ }^{\wedge}$ "ischemic preconditioning"]

\#6((isch?emic near/5 (condition or precondition* or postcondition $\left.{ }^{\star}\right)$ ) or IPC or RIC or RIPC):ti,ab,kw (Word variations have been searched) \#7((limb or vascular) near/5 (isch?emia or occlusi^)):ti,ab,kw (Word variations have been searched)

\#8[mh Sphygmomanometers]

\#9sphygmomanometer ${ }^{\star}:$ ti,ab,kw (Word variations have been searched)

\#10(blood pressure near/5 (cuff or device or monitor or meter or guage)):ti,ab,kw (Word variations have been searched)

\#11\{or \#5-\#10\}

$\# 12 \# 4$ and \#11

\section{Appendix 2. MEDLINE (Ovid) search strategy}

1. cerebrovascular disorders/ or basal ganglia cerebrovascular disease/ or exp brain ischemia/ or carotid artery diseases/ or carotid artery thrombosis/ or intracranial arterial diseases/or cerebral arterial diseases/or exp "intracranial embolism and thrombosis"/ or exp stroke/ 2. (isch?emi\$ adj6 (stroke\$ or apoplex\$ or cerebral vasc\$ or cerebrovasc\$ or cva or attack\$)).tw. 
3. ( (brain or cerebr\$ or cerebell\$ or vertebrobasil\$ or hemispher\$ or intracran\$ or intracerebral or infratentorial or supratentorial or middle cerebr\$ or mca\$ or anterior circulation) adj5 (isch?emi\$ or infarct\$ or thrombo $\$$ or emboli\$ or occlus\$ or hypoxi\$)).tw.

4. 1 or 2 or 3

5. ischemic postconditioning/ or exp ischemic preconditioning/

6. ((isch?emic adj5 (condition\$ or precondition\$ or postcondition\$)) or IPC or RIC or RIPC).tw.

7. ((limb or vascular) adj5 (isch?emia or occlusi\$)).tw.

8. exp Sphygmomanometers/

9. sphygmomanometer\$.tw.

10. (blood pressure adj5 (cuff or device or monitor or meter or guage)).tw.

11. or/5-10

12. Randomized Controlled Trials as Topic/

13. Random Allocation/

14. Controlled Clinical Trials as Topic/

15. control groups/

16. clinical trials as topic/ or clinical trials, phase i as topic/ or clinical trials, phase ii as topic/ or clinical trials, phase iii as topic/ or clinical trials, phase iv as topic/

17. double-blind method/

18. single-blind method/

19. Placebos/

20. placebo effect/

21. cross-over studies/

22. randomized controlled trial.pt.

23. controlled clinical trial.pt.

24. (clinical trial or clinical trial phase i or clinical trial phase ii or clinical trial phase iii or clinical trial phase iv).pt.

25. (random\$ or RCT or RCTs).tw.

26. (controlled adj5 (trial\$ or stud\$)).tw.

27. (clinical\$ adj5 trial\$).tw.

28. ((control or treatment or experiment\$ or intervention) adj5 (group $\$$ or subject\$ or patient\$)).tw.

29. ((control or experiment or conservative) adj5 (treatment or therapy or procedure or manage\$)).tw.

30. ((singl\$ or doubl\$ or tripl\$ or trebl\$) adj5 (blind\$ or mask\$)).tw.

31. (cross-over or cross over or crossover).tw.

32. (placebo $\$$ or sham).tw.

33. trial.ti.

34. (assign\$ or allocat\$).tw.

35. controls.tw.

36. or/12-35

37.4 and 11 and 36

\section{Appendix 3. Embase (Ovid) search strategy}

1. cerebrovascular disease/ or brain infarction/ or brain stem infarction/ or cerebellum infarction/ or exp brain ischemia/ or carotid artery disease/ or exp carotid artery obstruction/ or cerebral artery disease/ or exp cerebrovascular accident/ or exp occlusive cerebrovascular disease/ or stroke patient/

2. (isch?emi\$ adj5 (stroke\$ or apoplex\$ or cerebral vasc\$ or cerebrovasc\$ or cva)).tw.

3. ( (brain or cerebr $\$$ or cerebell\$ or vertebrobasil\$ or hemispher $\$$ or intracran $\$$ or intracerebral or infratentorial or supratentorial or middle cerebr\$ or mca $\$$ or anterior circulation or basilar artery or vertebral artery) adj5 (isch?emi\$ or infarct\$ or thrombo $\$$ or emboli\$ or occlus \$ or hypoxi\$)).tw.

4. 1 or 2 or 3

5. ischemic postconditioning/ or ischemic preconditioning/

6. ((isch?emic adj5 (condition\$ or precondition\$ or postcondition\$)) or IPC or RIC or RIPC).tw.

7. ((limb or vascular) adj5 (isch?emia or occlusi\$)).tw.

8. sphygmomanometer/

9. sphygmomanometer\$.tw.

10. (blood pressure adj5 (cuff or device or monitor or meter or guage)).tw.

11. or/5-10

12. Randomized Controlled Trial/ or "randomized controlled trial (topic)"/

13. Randomization/

14. Controlled clinical trial/ or "controlled clinical trial (topic)"/

15. control group/ or controlled study/

16. clinical trial/ or "clinical trial (topic)"/ or phase 1 clinical trial/ or phase 2 clinical trial/ or phase 3 clinical trial/ or phase $4 \mathrm{clinical}$ trial/

17. Crossover Procedure/

18. Double Blind Procedure/ 
19. Single Blind Procedure/ or triple blind procedure/

20. placebo/ or placebo effect/

21. (random\$ or RCT or RCTs).tw.

22. (controlled adj5 (trial\$ or stud\$)).tw.

23. (clinical\$ adj5 trial\$).tw.

24. ((control or treatment or experiment $\$$ or intervention) adj5 (group\$ or subject\$ or patient\$)).tw.

25 . (quasi-random $\$$ or quasi random $\$$ or pseudo-random $\$$ or pseudo random $\$$ ).tw.

26. ((control or experiment\$ or conservative) adj5 (treatment or therapy or procedure or manage\$)).tw.

27. ((singl\$ or doubl\$ or tripl\$ or trebl\$) adj5 (blind\$ or mask\$)).tw.

28. (cross-over or cross over or crossover).tw.

29. (placebo\$ or sham).tw.

30. trial.ti.

31. (assign\$ or allocat\$).tw.

32. controls.tw.

33. or/12-32

34. 4 and 11 and 33

\section{Appendix 4. Science Citation Index Expanded (SCI-EXPANDED) and Conference Proceedings Citation Index-Science} (CPCI-S)search strategy

\# $1 \mathrm{Tl}=$ (stroke or poststroke or post-stroke or cerebrovasc ${ }^{*}$ or brain vasc ${ }^{\star}$ or cerebral vasc* or cva* or apoplex ${ }^{\star}$ or SAH) Indexes=SCI-EXPANDED Timespan=1950-2018

\# $2 \mathrm{Tl}=$ (isch\$emic * conditioning)

Indexes=SCI-EXPANDED Timespan=1950-2018

\# 3 \#1 AND \#2

Indexes=SCI-EXPANDED Timespan=1950-2018

\section{Appendix 5. China Biological Medicine Database (CBM) search strategy}

$\# 1$ 脑缺血 OR 脑梗塞 OR 颈动脉疾病 OR 颈动脉血栓形成 OR 颈动脉血栓解剖 OR 颅内动脉疾病 OR 脑动脉疾病 OR 脑梗死 OR 颅内栓 塞和血栓形成 OR 缺血性卒中

\#2 主题词="脑梗死" [不加权: 扩展] OR "脑血管障碍"[不加权:扩展] OR "脑梗死" [不加权: 扩展] OR "脑梗死" [不加权: 扩展] OR "脑 干梗死"[不加权:扩展]

\#3 中风 OR CVA OR 脑血管意外

\#4 缺血 $\$$ 处理 OR IPC or RIC or RIPC OR 缺血 $\$$ 适应 OR 远隔缺血 $\$$ 处理 OR 远隔缺血 $\$$ 适应 OR 预处理

\#5 (\#3) OR (\#2) OR (\#1)

\#6 (\#5) AND (\#4) AND ( 临床试验[文献类型] OR 随机对照试验[文献类型] OR 多中心研究 [文献类型])

\section{Appendix 6. China Doctoral Dissertations Full-text Database (CDFD) search strategy}

$(S U=$ '缺血处理' ORSU = 'IPC' OR SU = 'RIC' OR SU = 'RIPC' OR SU = '缺血预处理 ' OR SU = '缺血适应' OR SU = '缺血预适应' OR SU = '缺 血后适应' ORSU = '缺血后处理' ORSU = '预处理' OR SU = '远隔缺血处理' ORSU = '远隔缺血适应' $)$ AND $(S U=$ '脑缺血 ' ORSU = '脑 梗塞 ' ORSU = '颈动脉疾病 ' OR SU = '颈动脉血栓形成 ' OR SU = '颈动脉血栓解剖' OR SU = '颅内动脉疾病 ' OR SU $=$ '脑动脉疾病 ' OR $\mathrm{SU}=$ '脑梗死' OR SU = '颅内栓塞和血栓形成' OR SU = '缺血性卒中 ' OR SU = '脑血栓形成' OR SU = '脑血管意外' OR SU = '脑中风 ' )

\section{Appendix 7. China Academic Journal Network Publishing Database (CAJD) search strategy}

$((S U=$ '缺血处理 ' OR SU = 'IPC' OR SU = 'RIC' OR SU = 'RIPC' OR SU = '缺血预处理' OR SU = '缺血适应' OR SU = '缺血预适应' OR SU = '缺血后适应' OR SU = '缺血后处理' OR SU = '预处理' OR SU = '远隔缺血处理' OR SU = '远隔缺血适应' $)$ AND (SU= '脑缺血 ' OR SU $=$ '脑梗塞' OR SU = '颈动脉疾病' OR SU = '颈动脉血栓形成 ' OR SU = '颈动脉血栓解剖' OR SU = '颅内动脉疾病' OR SU = '脑动脉疾 病' OR SU = '脑梗死' OR SU = '颅内栓塞和血栓形成' OR SU = '缺血性卒中' OR SU = '脑血栓形成' OR SU = '脑血管意外 ' OR SU = '脑 中风' ) ) NOT (SU = '基因' OR SU = '分子' OR SU = '大鼠' OR SU = '小鼠' OR SU = '动物 ')

\section{Appendix 8. World Health Organization (WHO) International Clinical Trials Registry Platform search strategy}

Ischemic *conditioning [Intervention] AND Stroke [Condition]

\section{Appendix 9. ClinicalTrials.gov search strategy}

ischaemic conditioning AND Cerebrovascular Disorders [DISEASE] 


\section{Appendix 10. Stroke Trials Registry search strategy}

("Ischemic Conditioning" or "Remote Ischemic Conditioning" or "Remote ischemic preconditioning stimulus" or "Remote ischemic preconditioning (RIPC)" or "Remote ischemic preconditioning" or "Remote Ischemic Pre-conditioning" or "Sham Remote Ischemic Preconditioning" or "Remote Ischemic Conditioning (using Sphygmomanometer)" or "Remote ischemic postconditioning" or "Remote ischemic perconditioning") [INTERVENTIONS] AND Stroke [CONDITIONS]

\section{Appendix 11. ISRCTN Registry search strategy}

Ischaemic * conditioning [Interventions] AND Stroke [Condition]

\section{WHAT'S NEW}

\begin{tabular}{lll}
\hline Date & Event & Description \\
\hline 28 September 2019 & Amended & $\begin{array}{l}\text { Two sections were amended: (1) the "Declarations of interest" } \\
\text { section; and (2) the "3 Embase (Ovid) search strategy" of Appen- } \\
\text { dices section (\#37 was updated as "4 and 11 and 36"). }\end{array}$ \\
\hline
\end{tabular}

\section{CONTRIBUTIONSOF AUTHORS}

WBZ and JZ assessed all trials for eligibility, extracted data, and assessed the risk of bias. RM performed literature searches of the Chinese language studies. MGS helped with the related trials selection and looked for the full texts. YCD and XMJ drafted the review text with input from the other authors and revised the manuscript. All review authors contributed to writing and revising this review.

\section{DECLARATIONS OF INTEREST}

Wenbo Zhao: none known.

Jing Zhang: none known.

Mordechai G Sadowsky: none known.

Ran Meng: none known.

Yuchuan Ding: none known.

Xunming Ji: is one of the inventors of the electric auto-control device that induces remote ischaemic conditioning. This device has been used in many studies, including the ones mentioned in this review. There is no conflict of interest in the conduct of this review. The ownership of the patent belongs to Xuanwu Hospital, Capital Medical University. Dr Ji does not participate in any commercial activities or profits related to the device.

\section{SOURCES OF SUPPORT}

\section{Internal sources}

- No sources of support supplied

\section{External sources}

- National Key R\&D Program of China (No. 2017YFC1308400), China.

Grant for the research of remote ischaemic conditioning for chronic cerebrovascular diseases

- Beijing Municipal Administration of Hospitals Incubating Program (Code:PX2018033), China.

Grants for the research of remote ischaemic conditioning for intracranial atherosclerosis

- Beijing Municipal Administration of Hospitals Clinical Medicine Development of Special Funding (ZYLX201706), China.

Grant for the research of ischaemic stroke

\section{DIFFERENCES BETWEEN PROTOCOLANDREVIEW}

We had planned to include participants undergoing cardiovascular intervention therapies, coronary artery bypass grafting, and any other type of cardiac surgery. However, we found that cardioembolism is the main cause of stroke in the above participants, which is closely related with operative procedures. In conventional studies focusing on ischaemic stroke, the above participants were excluded. In addition, all cardiac studies investigated the effects of RIC on cardiovascular events, plasma biomarkers or imaging outcomes; many of them did 
not report cerebrovascular events if they occurred. Some bias may be caused unintentionally if the above participants were included. Therefore, we did not include these participants this review.

We had intended to performed subgroup analyses to investigate heterogeneity by analysing differences in the number of RIC cycles and their length, and the position and length of RIC treatment. However, in this review only seven trials were eligible for analysis, including three studies for the analysis of RIC for preventing ischaemic stroke, and another four studies for the analysis of RIC for treating ischaemic stroke. Given the limited number of included studies, there were not enough data for subgroup analyses.

We had intended to perform sensitivity analyses if the results were substantially heterogeneous. However, we did not perform any sensitivity analyses because of the limited number of included studies, and we found no substantial heterogeneity in this review.

We had intended to include 'quality of life' as a outcome in the 'Summary of findings' table. However, no included measured this outcome. Instead, we included 'stroke severity', which has been assessed by NHISS scores or infarct volume in several studies, as a outcome in the 'Summary of findings' table.

\section{IN DEX TERMS}

\section{Medical Subject Headings (MeSH)}

${ }^{\star}$ Neuroprotection; Bias; Cerebral Arterial Diseases [complications]; Extremities [blood supply]; Ischemic Preconditioning [ ${ }^{\star}$ methods] [mortality]; Randomized Controlled Trials as Topic; Stroke [mortality] [prevention \& control] [therapy]; Thrombolytic Therapy [mortality]

\section{MeSH check words}

Humans 